\title{
On the Performance Gain of NOMA over OMA in Uplink Communication Systems
}

\author{
Zhiqiang Wei, Lei Yang, Derrick Wing Kwan Ng, Jinhong Yuan, and Lajos Hanzo
}

(Invited Paper)

\begin{abstract}
In this paper, we investigate and reveal the ergodic sum-rate gain (ESG) of non-orthogonal multiple access (NOMA) over orthogonal multiple access (OMA) in uplink cellular communication systems. A base station equipped with a single-antenna, with multiple antennas, and with massive antenna arrays is considered both in single-cell and multi-cell deployments. In particular, in single-antenna systems, we identify two types of gains brought about by NOMA: 1) a large-scale near-far gain arising from the distance discrepancy between the base station and users; 2) a small-scale fading gain originating from the multipath channel fading. Furthermore, we reveal that the largescale near-far gain increases with the normalized cell size, while the small-scale fading gain is a constant, given by $\gamma=0.57721$ nat $/ \mathbf{s} / \mathbf{H z}$, in Rayleigh fading channels. When extending singleantenna NOMA to $M$-antenna NOMA, we prove that both the large-scale near-far gain and small-scale fading gain achieved by single-antenna NOMA can be increased by a factor of $M$ for a large number of users. Moreover, given a massive antenna array at the base station and considering a fixed ratio between the number of antennas, $M$, and the number of users, $K$, the ESG of NOMA over OMA increases linearly with both $M$ and $K$. We then further extend the analysis to a multi-cell scenario. Compared to the single-cell case, the ESG in multi-cell systems degrades as NOMA faces more severe inter-cell interference due to the non-orthogonal transmissions. Besides, we unveil that a large cell size is always beneficial to the ergodic sum-rate performance of NOMA in both single-cell and multi-cell systems. Numerical results verify the accuracy of the analytical results derived and confirm the insights revealed about the ESG of NOMA over OMA in different scenarios.
\end{abstract}

Index Terms-Non-orthogonal multiple access, ergodic sumrate gain, large-scale near-far gain, small-scale fading gain, inter-

Manuscript received March 4, 2019; revised July 14, 2019 and October 2, 2019; accepted October 6, 2019. This work was partially supported by the Australia Research Council Discovery Project DP190101363 and Linkage Projects (LP160100708 and LP170101196) and the National Natural Science Foundation of China under contract No. 61971403 and the Special Presidential Foundation of Technology and Engineering Center for Space Utilization of the Chinese Academy of Sciences under Project CSU-QZKT2018-16. D. W. K. Ng is supported by funding from the UNSW Digital Grid Futures Institute, UNSW, Sydney, under a cross-disciplinary fund scheme and by the Australian Research Council's Discovery Early Career Researcher Award (DE170100137). L. Hanzo would like to acknowledge the financial support of the Engineering and Physical Sciences Research Council projects EP/Noo4558/1, EP/PO34284/1, COALESCE, of the Royal Society's Global Challenges Research Fund Grant as well as of the European Research Council's Advanced Fellow Grant QuantCom. The conference version of this paper has been presented at the IEEE Globecom 2018 [1]. The editor coordinating the review of this paper and approving it for publication was Prof. D. I. Kim.

Zhiqiang Wei, Derrick Wing Kwan Ng, and Jinhong Yuan are with the School of Electrical Engineering and Telecommunications, the University of New South Wales, Australia (email: zhiqiang.wei@unsw.edu.au; w.k.ng@unsw.edu.au; j.yuan@unsw.edu.au).

Lei Yang is with the Technology and Engineering Center for Space Utilization, Chinese Academy of Science (email: yang.lei@csu.ac.cn).

Lajos Hanzo is with the School of Electronics and Computer Science, University of Southampton, Southampton, UK (email: 1h@ecs.soton.ac.uk). cell interference.

\section{INTRODUCTION}

The networked world we live in has revolutionized our daily life. Wireless communications has become one of the disruptive technologies and it is one of the best business opportunities of the future [2], [3]. In particular, the development of wireless communications worldwide fuels the massive growth in the number of wireless communication devices and sensors for emerging applications such as smart logistics \& transportation, environmental monitoring, energy management, safety management, and industry automation, just to name a few. It is expected that in the Internet-of-Things (IoT) era [4], there will be 50 billion wireless communication devices connected worldwide with a connection density up to a million devices per $\mathrm{km}^{2}$ [5], [6]. The massive number of devices and explosive data traffic pose challenging requirements, such as massive connectivity [7] and ultra-high spectral efficiency for future wireless networks [2], [3]. As a result, compelling new technologies, such as massive multiple-input multiple-output (MIMO) [8]-[11], non-orthogonal multiple access (NOMA) [12]-[16], and millimeter wave (mmWave) communications [17]-[21] etc. have been proposed to address the aforementioned issues. Among them, NOMA has drawn significant attention both in industry and in academia as a promising multiple access technique. The principle of powerdomain NOMA is to exploit the users' power difference for multiuser multiplexing together with superposition coding at the transmitter, while applying successive interference cancelation (SIC) at the receivers for alleviating the inter-user interference (IUI) [14]. In fact, the industrial community has proposed up to 16 various forms of NOMA as the potential multiple access schemes for the forthcoming fifth-generation (5G) networks [22].

Compared to the conventional orthogonal multiple access (OMA) schemes [23]-[25], NOMA allows users to simultaneously share the same resource blocks and hence it is beneficial for supporting a large number of connections in spectrally efficient communications. The concept of nonorthogonal transmissions dates back to the 1990s, e.g. [26], [27], which serves as a foundation for the development of the power-domain NOMA. Indeed, NOMA schemes relying on non-orthogonal spreading sequences have led to popular code division multiple access (CDMA) arrangements, even though eventually the so-called orthogonal variable spreading factor (OVSF) code was selected for the global third-generation (3G) wireless systems [28]-[31]. To elaborate a little further, 
the spectral efficiency of CDMA was analyzed in [28]. In [29], the authors compared the benefits and deficiencies of three typical CDMA schemes: single-carrier direct-sequence CDMA (SC DS-CDMA), multicarrier CDMA (MC-CDMA), and multicarrier DS-CDMA (MC DS-CDMA). Furthermore, a comparative study of OMA and NOMA was carried out in [31]. It has been shown that NOMA possesses a spectralpower efficiency advantage over OMA [31] and this theoretical gain can be realized with the aid of the interleave division multiple access (IDMA) technique proposed in [32]. Despite the initial efforts on the study of NOMA, the employment of NOMA in practical systems has been developing relatively slowly due to the requirement of sophisticated hardware for its implementation. Recently, NOMA has rekindled the interests of researchers as a benefit of the recent advances in signal processing and silicon technologies [33], [34]. However, existing contributions, e.g. [26], [27], [31], have mainly focused their attention on the NOMA performance from the information theoretical point of view, such as its capacity region [26], [27] and power region [31]. The recent work in [35] intuitively explained the source of performance gain attained by NOMA over OMA via simulations. The authors of [36], [37] surveyed the state-of-the-art research on NOMA and offered a high-level discussion of the challenges and research opportunities for NOMA systems. Nevertheless, to the best of our knowledge, there is a paucity of literature on the comprehensive analysis of the achievable ergodic sum-rate gain (ESG) of NOMA over OMA relying on practical signal detection techniques. Furthermore, the ESG of NOMA over OMA in different practical scenarios, such as single-antenna, multi-antenna, and massive antenna array aided systems relying on single-cell or multi-cell deployments has not been compared in the open literature.

As for single-antenna systems, several authors have analyzed the performance of NOMA from different perspectives, e.g. [38]-[41]. More specifically, based on the achievable rate region, Xu et al. proved in [38] that NOMA outperforms time division multiple access (TDMA) with a high probability in terms of both its overall sum-rate and the individual user-rate. Furthermore, the ergodic sum-rate of singleinput single-output NOMA (SISO-NOMA) was derived and the performance gain of SISO-NOMA over SISO-OMA was demonstrated via simulations by Ding et al. [39]. Upon relying on their new dynamic resource allocation design, Chen et al. [40] proved that SISO-NOMA always outperforms SISOOMA using a rigorous optimization technique. In [41], Yang et $a l$. analyzed the outage probability degradation and the ergodic sum-rate of SISO-NOMA systems by taking into account the impact of partial channel state information (CSI). As a further development, efficient resource allocation was designed for NOMA systems by Sun et al. [42] as well as by Wei et al. [43] under the assumptions of perfect CSI and imperfect CSI, respectively. The simulation results in [42] and [43] demonstrated the performance gain of NOMA over OMA in terms of its spectral efficiency and power efficiency, respectively. The aforementioned contributions studied the performance of NOMA systems or discussed the superiority of NOMA over OMA in different contexts. However, the analytical results quantifying the ESG of SISO-NOMA over SISO-OMA has not been reported at the time of writing. More importantly, the source of the performance gain of NOMA over OMA has not been well understood and the impact of specific system parameters on the ESG, such as the number of NOMA users, the signal-to-noise ratio (SNR), and the cell size, have not been revealed in the open literature.

To achieve a higher spectral efficiency, the concept of NOMA has also been amalgamated with multi-antenna systems, resulting in the notion of multiple-input multiple-output NOMA (MIMO-NOMA) [52], for example, by invoking the signal alignment technique of Ding et al. [44] and the quasidegradation-based precoding design of Chen et al. [45]. Furthermore, through building up the bounds for MIMO-NOMA, Zeng et al. [46] and Liu et al. [47] have proven that MIMONOMA outperforms MIMO-OMA in terms of both sumrate and ergodic sum-rate. However, no analytical expressions were provided in [46], [47] for revealing important insights about the performance gain of NOMA over OMA in multiantenna systems. Although the performance gain of MIMONOMA over MIMO-OMA has indeed been shown in [44], [45] with the aid of simulations, the performance gain due to additional antennas has not been quantified mathematically. Moreover, how the ESG of NOMA over OMA increases upon upgrading the system from having a single antenna to multiple antennas is still an open problem at the time of writing, which deserves our efforts to explore. The answers to these questions can shed light on the practical implementation of NOMA in future wireless networks. On the other hand, there are only some preliminary results on applying the NOMA principle to massive-MIMO systems. For instance, Zhang et al. [53] investigated the outage probability of massiveMIMO-NOMA ( $m$ MIMO-NOMA). Furthermore, Ding and Poor [48] analyzed the outage performance of $m$ MIMONOMA relying on realistic limited feedback and demonstrated a substantial performance improvement for $m$ MIMO-NOMA over $m$ MIMO-OMA. Upon extending NOMA to a mmWave massive-MIMO system, the capacity attained in the high-SNR regime and low-SNR regime were analyzed by Zhang et al. [54]. Yet, the ESG of $m$ MIMO-NOMA over $m$ MIMO-OMA remains unknown and the investigation of $m$ MIMO-NOMA has the promise attaining NOMA gains in large-scale systems in the networks of the near future.

On the other hand, although single-cell NOMA has received significant research attention [38]-[45], [48], [53], [54], the performance of NOMA in multi-cell scenarios remains unexplored but critically important for practical deployment, where the inter-cell interference becomes a major obstacle [55]. Centralized resource optimization of multi-cell NOMA was proposed by You et al. in [49], while a distributed power control scheme was studied in [50]. The transmit precoder design of MIMO-NOMA aided multi-cell networks designed for maximizing the overall sum throughput was developed by Nguyen et al. [51] and a computationally efficient algorithm was proposed for achieving a locally optimal solution. Despite the fact that the simulation results provided by [49]-[51], [55] have demonstrated a performance gain for applying NOMA in multi-cell cellular networks, the analytical results quantifying 
TABLE I

COMParison of This Work With Literature For the Results of PERFormance Gain of NOMA OVER OMA

\begin{tabular}{|c|c|c|c|c|c|c|c|c|c|}
\hline System setup & Main results & 35 & [39] & [40 & [44], [45] & [46], [47] & {$[48$} & [49]- & This work \\
\hline $\begin{array}{c}\text { Single-antenna } \\
\text { single-cell systems }\end{array}$ & $\begin{array}{l}\text { Outage probability } \\
\text { Proof of superiority } \\
\text { Ergodic sum-rate } \\
\text { Ergodic sum-rate gain } \\
\text { Numerical results }\end{array}$ & $\checkmark$ & $\checkmark$ & $\begin{array}{l}\checkmark \\
\checkmark\end{array}$ & & & & & $\begin{array}{l}\checkmark \\
\checkmark \\
\checkmark \\
\checkmark\end{array}$ \\
\hline $\begin{array}{c}\text { Multi-antenna } \\
\text { single-cell systems }\end{array}$ & $\begin{array}{l}\text { Outage probability } \\
\text { Proof of superiority } \\
\text { Ergodic sum-rate } \\
\text { Ergodic sum-rate gain } \\
\text { Numerical results }\end{array}$ & & & & $\checkmark$ & $\begin{array}{l}\checkmark \\
\checkmark\end{array}$ & & & $\begin{array}{l}\checkmark \\
\checkmark \\
\checkmark \\
\checkmark\end{array}$ \\
\hline $\begin{array}{l}\text { Massive-MIMO } \\
\text { single-cell systems }\end{array}$ & $\begin{array}{l}\text { Outage probability } \\
\text { Proof of superiority } \\
\text { Ergodic sum-rate } \\
\text { Ergodic sum-rate gain } \\
\text { Numerical results }\end{array}$ & $\checkmark$ & & & & & $\checkmark$ & & $\begin{array}{l}\checkmark \\
\checkmark \\
\checkmark \\
\checkmark\end{array}$ \\
\hline Multi-cell systems & $\begin{array}{l}\text { Outage probability } \\
\text { Proof of superiority } \\
\text { Ergodic sum-rate } \\
\text { Ergodic sum-rate gain } \\
\text { Numerical results }\end{array}$ & $\checkmark$ & & & & & & $\checkmark$ & $\begin{array}{l}\checkmark \\
\checkmark \\
\checkmark \\
\checkmark \\
\checkmark\end{array}$ \\
\hline
\end{tabular}

the ESG of NOMA over OMA for multi-cell systems relying on single-antenna, multi-antenna, and massive-MIMO arrays at the BSs have not been reported in the open literature. Furthermore, the performance gains disseminated in the literature have been achieved for systems having a high transmit power or operating in the high-SNR regime. However, a high transmit power inflicts a strong inter-cell interference, which imposes a challenge for the design of inter-cell interference management. Therefore, there are many practical considerations related to the NOMA principle in multi-cell systems, while have to be investigated.

In summary, the comparison of our work with the most pertinent existing contributions in the literature is shown in Table I. Although the existing treatises have investigated the system performance of NOMA from different perspectives, such as the outage probability [39], [44], [45], [48] and the ergodic sum-rate [39], in various specifically considered system setups, no unified analysis has been published to discuss the performance gain of NOMA over OMA. To fill this gap, our work offers a unified analysis on the ergodic sum-rate gain of NOMA over OMA in single-antenna, multiantenna, and massive-MIMO systems with both single-cell and multi-cell deployments.

This paper aims for providing answers to the above open problems and for furthering the understanding of the ESG of NOMA over OMA in the uplink of communication systems. To this end, we carry out the unified analysis of ESG in singleantenna, multi-antenna and massive-MIMO systems. We first focus our attention on the ESG analysis in single-cell systems and then extend our analytical results to multi-cell systems by taking into account the inter-cell interference (ICI). We quantify the ESG of NOMA over OMA relying on practical signal reception schemes at the base station for both NOMA as well as OMA and unveil its behaviour under different scenarios. Our simulation results confirm the accuracy of our performance analyses and provide some interesting insights, which are summarized in the following:

- In all the cases considered, a high ESG can be achieved by NOMA over OMA in the high-SNR regime, but the ESG vanishes in the low-SNR regime.

- In the single-antenna scenario, we identify two types of gains attained by NOMA and characterize their different behaviours. In particular, we show that the largescale near-far gain achieved by exploiting the distancediscrepancy between the base station and users increases with the cell size, while the small-scale fading gain is given by an Euler-Mascheroni constant [56] of $\gamma=0.57721$ nat/s/Hz in Rayleigh fading channels.

- When applying NOMA in multi-antenna systems, compared to the MIMO-OMA utilizing zero-forcing detection, we analytically show that the ESG of SISO-NOMA over SISO-OMA can be increased by $M$-fold, when the base station is equipped with $M$ antennas and serves a sufficiently large number of users $K$.

- Compared to MIMO-OMA utilizing a maximal ratio combining (MRC) detector, an $(M-1)$-fold degrees of freedom (DoF) gain can be achieved by MIMO-NOMA. In particular, the ESG in this case increases linearly with the system's SNR quantified in $\mathrm{dB}$ with a slope of $(M-1)$ in the high-SNR regime.

- For massive-MIMO systems with a fixed ratio between the number of antennas, $M$, and the number of users, $K$, i.e., $\delta=\frac{M}{K}$, the ESG of $m$ MIMO-NOMA over $m$ MIMOOMA increases linearly with both $K$ and $M$ using MRC detection.

- In practical multi-cell systems operating without joint cell signal processing, the ESG of NOMA over OMA is degraded due to the existence of ICI, especially for a small cell size with a dense cell deployment. Furthermore, no DoF gain can be achieved by NOMA in multi- 


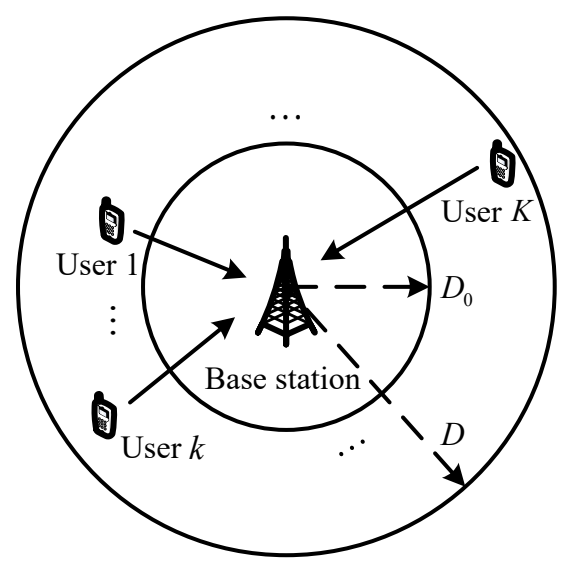

Fig. 1. The system model of the single-cell uplink communication with one base station and $K$ users.

cell systems due to the lack of joint multi-cell signal processing to handle the ICI. In other words, all the ESGs of NOMA over OMA in single-antenna, multi-antenna, and massive-MIMO multi-cell systems saturate in the high-SNR regime.

- For both single-cell and multi-cell systems, a large cell size is always beneficial to the performance of NOMA. In particular, in single-cell systems, the ESG of NOMA over OMA is increased for a larger cell size due to the enhanced large-scale near-far gain. For multi-cell systems, a larger cell size reduces the ICI level, which prevents a severe ESG degradation.

The notations used in this paper are as follows. Boldface capital and lower case letters are reserved for matrices and vectors, respectively. $(\cdot)^{\mathrm{T}}$ denotes the transpose of a vector or matrix and $(\cdot)^{\mathrm{H}}$ denotes the Hermitian transpose of a vector or matrix. $\mathbb{C}^{M \times N}$ represents the set of all $M \times N$ matrices with complex entries. $|\cdot|$ denotes the absolute value of a complex scalar or the determinant of a matrix, $\|\cdot\|$ denotes Euclidean norm of a complex vector, $\lceil\cdot\rceil$ is the ceiling function which returns the smallest integer greater than the input value, and $\mathrm{E}_{x}\{\cdot\}$ denotes the expectation over the random variable $x$. The circularly symmetric complex Gaussian distribution with mean $\mu$ and variance $\sigma^{2}$ is denoted by $\mathcal{C N}\left(\mu, \sigma^{2}\right)$.

\section{SYSTEM MODEL}

\section{A. System Model}

We first consider the uplink ${ }^{1}$ of a single-cell ${ }^{2}$ NOMA system with a single base station (BS) supporting $K$ users, as shown in Fig. 1. The cell is modeled by a pair of two concentric ringshaped discs. As a distance-based channel model is adopted in this work, the inner radius $D_{0}$ is introduced to model the minimum propagation path loss in the empirical path loss model [58], while the outer radius $D$ denotes the cell size. The

\footnotetext{
${ }^{1}$ We restrict ourselves to the uplink NOMA communications [57], as advanced signal detection/decoding algorithms of NOMA are more affordable at the base station.

${ }^{2}$ We first focus on the ESG analysis for single-cell systems, which serves as a building block for the analyses for multi-cell systems presented in Section $\mathrm{VI}$
}

$\mathrm{BS}$ is located at the center and all the $K$ users are scattered uniformly within the two concentric ring-shaped discs. For the NOMA scheme, all the $K$ users are multiplexed on the same frequency band and time slot, while for the OMA scheme, $K$ users utilize the frequency or time resources orthogonally. Without loss of generality, we consider frequency division multiple access (FDMA) as a typical OMA scheme.

In this paper, we consider three typical types of communication systems:

- SISO-NOMA and SISO-OMA: the BS is equipped with a single-antenna $(M=1)$ and all the $K$ users also have a single-antenna.

- MIMO-NOMA and MIMO-OMA: the BS is equipped with a multi-antenna array $(M>1)$ and all the $K$ users have a single-antenna associated with $K>M$.

- Massive MIMO-NOMA (mMIMO-NOMA) and massiveMIMO-OMA ( $m$ MIMO-OMA): the BS is equipped with a large-scale antenna array $(M \rightarrow \infty)$, while all the $K$ users are equipped with a single antenna, associated with $\frac{M}{K}=\delta<1$, i.e., the number of antennas $M$ at the BS is lower than the number of users $K$, but with a fixed ratio of $\delta<1$.

\section{B. Signal and Channel Model}

The signal received at the $\mathrm{BS}$ is given by

$$
\mathbf{y}=\sum_{k=1}^{K} \mathbf{h}_{k} \sqrt{p_{k}} x_{k}+\mathbf{v},
$$

where $\mathbf{y} \in \mathbb{C}^{M \times 1}, p_{k}$ denotes the power transmitted by user $k, x_{k}$ is the normalized modulated symbol of user $k$ with $\mathrm{E}\left\{\left|x_{k}\right|^{2}\right\}=1$, and $\mathbf{v} \sim \mathcal{C N}\left(\mathbf{0}, N_{0} \mathbf{I}_{M}\right)$ represents the additive white Gaussian noise (AWGN) at the BS with zero mean and covariance matrix of $N_{0} \mathbf{I}_{M}$. To emphasize the impact of the number of users $K$ on the performance gain of NOMA over OMA, we fix the total power consumption of all the uplink users and thus we have

$$
\sum_{k=1}^{K} p_{k} \leq P_{\max }
$$

where $P_{\max }$ is the maximum total transmit power for all the users. In the literature [35], [59], [60], the sum-power constraint is commonly adopted to simplify the performance analysis for uplink communications. More importantly, in this work, the sum-power constraint is imposed to emphasize the impact of the number of users, $K$, on the performance gain of NOMA over OMA. In contrast, the system total transmit power increases linearly with $K$ for a fixed maximum individual power, which prevents us to unveil the insights on the relationship between $K$ and the ESG. In practice, the sum-power constraint can be adopted to limit the excessive radiation to fulfill some practical regulations on spectrum mask. Specifically, in our considered system model, no matter how many users are served in a single-cell, their total transmit power should be smaller than $P_{\max }$ to avoid a severe ICI, i.e., $\sum_{k=1}^{K} p_{k} \leq P_{\max }$ 
The uplink (UL) channel vector between user $k$ and the BS is modeled as

$$
\mathbf{h}_{k}=\frac{\mathbf{g}_{k}}{\sqrt{1+d_{k}^{\alpha}}},
$$

where $\mathbf{g}_{k} \in \mathbb{C}^{M \times 1}$ denotes the Rayleigh fading coefficients, i.e., $\mathbf{g}_{k} \sim \mathcal{C N}\left(\mathbf{0}, \mathbf{I}_{M}\right), d_{k}$ is the distance between user $k$ and the BS in the unit of meter, and $\alpha$ represents the path loss exponen 3 . We denote the UL channel matrix between all the $K$ users and the BS by $\mathbf{H}=\left[\mathbf{h}_{1}, \ldots, \mathbf{h}_{K}\right] \in \mathbb{C}^{M \times K}$. Note that the system model in (1) and the channel model in (3) include the cases of single-antenna and massive-MIMO aided BS associated with $M=1$ and $M \rightarrow \infty$, respectively. For instance, when $M=1, h_{k}=\frac{g_{k}}{\sqrt{1+d_{k}^{\alpha}}}$ denotes the corresponding channel coefficient of user $k$ in single-antenna systems. We assume that the channel coefficients are independent and identically distributed (i.i.d.) over all the users and antennas 4 Since this paper aims for providing some insights on the performance gain of NOMA over OMA, we assume that perfect UL CSI knowledge is available at the BS for coherent detection, which is commonly adopted in the literature [39], [44], [45], [62], 63].

Remark 1: In this paper, as a first attempt to unveil fundamental insights on the performance gain of NOMA over OMA, we consider an ideal case which we model the channel coefficients for all the users and antennas as i.i.d. random variables. In practice, spatial channel correlation does exists due to the antenna coupling and/or sparse propagation environment. However, it is challenging to analyze the performance gain of NOMA over OMA when channel correlation exists, which deserves a new work to be considered in the future. Nevertheless, in the following, the impacts of the channel correlation in both the antenna and the user domain are briefly discussed.

When channel correlation exists among receiving antennas at the BS, the received signals only span a subspace with a dimension smaller than the number of antennas $M$. At the same time, the spatial correlation among antennas reduces the system DoF [64] as we focus on $K \geq M$ in the considered massive MIMO communication system. Note that NOMA is suitable for a DoF-limited system as it allows multiple users to share one DoF with power domain multiplexing [13]. Therefore, the channel correlation in antenna domain favors NOMA and is potential to increase the performance gain of NOMA over OMA.

On the other hand, when there are channel correlations among users, IUI becomes more severe for both NOMA and OMA in both single-cell and multi-cell systems. However,

\footnotetext{
${ }^{3}$ In this paper, we ignore the impact of shadowing to simplify our performance analysis. Note that, shadowing only introduces an additional power factor to $\mathbf{g}_{k}$ in the channel model in (3). Although the introduction of shadowing may change the resulting channel distribution of $\mathbf{h}_{k}$, the distancebased channel model is sufficient to characterize the large-scale near-far gain exploited by NOMA, as will be discussed in this paper.

${ }^{4}$ We note that the channel gains of the users ordered for ensuing SIC operation are generally correlated according to order statistics theory [61]. However, as demonstrated in our performance analysis, we do not need to consider the channel correlation imposed by channel ordering. A detailed explanation on adopting independently distributed channels in our analysis is given in Appendix G.
}

OMA is very sensitive to IUI, while NOMA is in favor of interference-limited systems, owing to the execution of SIC detection. Therefore, exploiting the channel correlation among users is also a potential direction to enhance the performance gain of NOMA over OMA.

Remark 2: In practice, various uplink channel estimation schemes can be applied to achieve highly accurate CSI, e.g. the least square and the minimum mean square error (MMSE) methods [65]. In particular, each user transmits its pilot sequence to the BS and channel estimation is executed at the BS. Hence, we assume that perfect CSI is available at the BS for signal detection.

Furthermore, we would like to discuss the impact of CSI error on the performance gain of NOMA over OMA, as the CSI error is usually inevitable in practice. In particular, the CSI error for signal detection causes the non-removable residual interference [41], [66], which deteriorates the performance of both NOMA and OMA systems. For OMA schemes, as shown in Equations (5), 26, 29), and (46) in the latter part of this paper, the achievable data rate of user $k$ is only affected by its own CSI error. On the other hand, as shown in Equation (3) in our previous work [66], the achievable data rate of user $k$ is affected by the CSI error at the BS from not only user $k$ itself but also the previously decoded users $\{1,2, \ldots, k-1\}$. In fact, during SIC detection, the interference signals of previously decoded users cannot be completely eliminated due to the CSI error. Therefore, NOMA is generally more sensitive to the CSI error due to the accumulation of residual interference. As a result, the performance gain of NOMA over OMA might be redeemed with the existence of CSI error. However, as a first attempt to investigate the ESG, we focus on the case of perfect CSI and the study for the case of imperfect CSI will be considered in our future work.

\section{Signal Detection and Resource Allocation Strategy}

To facilitate our performance analyses, we focus our attention on the following efficient signal detection and practical resource allocation strategies.

1) Signal detection: The signal detection techniques adopted in this paper for NOMA and OMA systems are shown in Table [I] which are detailed in the following.

For SISO-NOMA, we adopt the commonly used successive interference cancelation (SIC) receiver [67] at the BS, since its performance approaches the capacity of single-antenna systems [27]. On the other hand, given that all the users are separated orthogonally by different frequency subbands for SISO-OMA, the simple single-user detection (SUD) technique can be used to achieve the optimal performance.

For MIMO-NOMA, the MMSE-based successive interference cancelation (MMSE-SIC) constitutes an appealing receiver algorithm, since its performance approaches the capacity [27] at an acceptable computational complexity for a finite number of antennas $M$ at the BS. On the other hand, two types of signal detection schemes are considered for MIMOOMA, namely FDMA zero forcing (FDMA-ZF) and FDMA maximal ratio combining (FDMA-MRC). Exploiting the extra spatial degrees of freedom (DoF) attained by multiple antennas 
TABLE II

SignAl DeteCtion TeChNiQUeS For NOMA AND OMA Systems

\begin{tabular}{cc|cc}
\hline NOMA system & Reception technique & OMA system & Reception technique \\
\hline SISO-NOMA & SIC & SISO-OMA & FDMA-SUD \\
MIMO-NOMA & MMSE-SIC & MIMO-OMA & FDMA-ZF, FDMA-MRC \\
$m$ MIMO-NOMA & MRC-SIC & mMIMO-OMA & FDMA-MRC \\
\hline
\end{tabular}

at the BS, ZF can be used for multi-user detection (MUD), as its achievable rate approaches the capacity in the high-SNR regime [27]. In particular, all the users are categorized into $G=K / M$ group $5^{5}$ with each group containing $M$ users. Then, ZF is utilized for handling the inter-user interference (IUI) within each group and FDMA is employed to separate all the $G$ groups on orthogonal frequency subbands. In the low-SNR regime, the performance of ZF fails to approach the capacity [27], thus a simple low-complexity MRC scheme is adopted for single user detection on each frequency subband. We note that there is only a single user in each frequency subband of our considered FDMA-MRC aided MIMO-OMA systems, i.e., no user grouping.

With a massive number of UL receiving antennas employed at the BS, we circumvent the excessive complexity of matrix inversion involved in ZF and MMSE detection by adopting the low-complexity MRC-SIC detection [66] for $m$ MIMONOMA systems and the FDMA-MRC scheme for $m$ MIMOOMA systems. Given the favorable propagation property of massive-MIMO systems [68], the orthogonality among the channel vectors of multiple users holds fairly well, provided that the number of users is sufficiently smaller than the number of antennas. Therefore, we can assign $W \ll M$ users to every frequency subband and perform the simple MRC detection while enjoying negligible IUIs in each subband. In this paper, we consider a fixed ratio between the group size and the number of antennas, namely, $\varsigma=\frac{W}{M} \ll 1$, and assume that the above-mentioned favorable propagation property holds under the fixed ratio $\varsigma$ considered.

Remark 3: As observed in Table II, the main difference in detection complexity between NOMA and OMA schemes is the SIC detection ${ }^{6}$ in all the considered scenarios, which introduces an extra detection complexity and delay for NOMA at the BS. In particular, SIC detection is essentially a multi-stage procedure to successively decode, regenerate, and subtract the signal of each NOMA user at the BS [14]. According to [69], both the complexity and the delay of SIC detection grows linearly with respect to (w.r.t.) the number of users $K$. On the other hand, as we assume the same total transmit power for both NOMA and OMA schemes in all the considered scenarios for fair comparison, the non-negative ESGs has implicitly shown an increased power efficiency of NOMA over OMA, i.e., the ergodic sum-rate achieved per Watt of transmit power. If the circuit power consumption for SIC detection at the BS dominates the total system power consumption, the power

\footnotetext{
${ }^{5}$ Without loss of generality, we consider the case with $G$ as an integer in this paper.

${ }^{6}$ Compared to FDMA-MRC for MIMO-OMA, an additional computational complexity $\mathcal{O}\left(M^{3}\right)$ is required for MIMO-NOMA due to the channel inversion in MMSE-SIC, apart from that of the SIC detection of MIMONOMA.
}

efficiency might be decreased. This is due to the fact that as $K \rightarrow \infty$, the ESG of NOMA over OMA becomes saturated as shown in Fig. 5, while the power consumption of SCI detection linearly increases w.r.t. $K$.

2) Resource allocation strategy: To facilitate our analytical study in this paper, we consider an equal resource allocation strategy for both NOMA and OMA schemes. In particular, equal power allocation is adopted for NOMA schemes 7 . On the other hand, equal power and frequency allocation is adopted for OMA schemes. As shown in the literature [43], [62], power control is usually adopted to potentially improve the performance gain of NOMA. Specifically, power control can increase the channel discrepancies between users which facilitates the exploitation of power domain multiplexing. However, traditional centralized uplink power control approaches [71], [72] may not be practical and scalable due to the required tremendous system overhead, particular for uplink NOMA systems serving a larger number of users. Therefore, distributed power control approaches [73], [74] were proposed for uplink NOMA to improve the scalability of the system w.r.t. the number of users. In contrast, our work focuses on investigating the source of performance gain of NOMA over OMA and the impact of specific system parameters on the ESG, such as the number of users, the number of antennas, the system SNR, and the cell size. To isolate the impact of resource allocation design on the performance gain of NOMA over OMA, we consider an equal power allocation for both NOMA and OMA schemes ${ }^{8}$ In addition, any adaptive power control strategy, either centralized or distributed approach, depends on the availability of global or local instantaneous channel state information. As a result, adopting an adaptive power control can complicate the performance analysis and hence we adopt an equal power allocation strategy to simplify the performance analysis and insights investigation. In practice, equal resource allocation is a typical selected strategy for applications bearing only a limited system overhead, e.g. machine-type communications (MTC) [6], [75].

We note that beneficial user grouping design is important for the MIMO-OMA system relying on FDMA-ZF and for the $m$ MIMO-OMA system using FDMA-MRC. In general, finding the optimal user grouping strategy is an NP-hard problem and the performance analysis based on the optimal user grouping strategy is generally intractable. Furthermore, the optimal SIC detection order of NOMA in multi-antenna and massiveMIMO systems is still an open problem in the literature, since the channel gains on different antennas are usually diverse.

\footnotetext{
${ }^{7}$ As shown in $70 \mid$, allocating a higher power to the user with the worse channel is not necessarily required in NOMA [70].

${ }^{8}$ Note that, even a fixed but imbalanced power allocation strategy may contribute to the ESG of NOMA over OMA and thus may bias the obtained insights.
} 


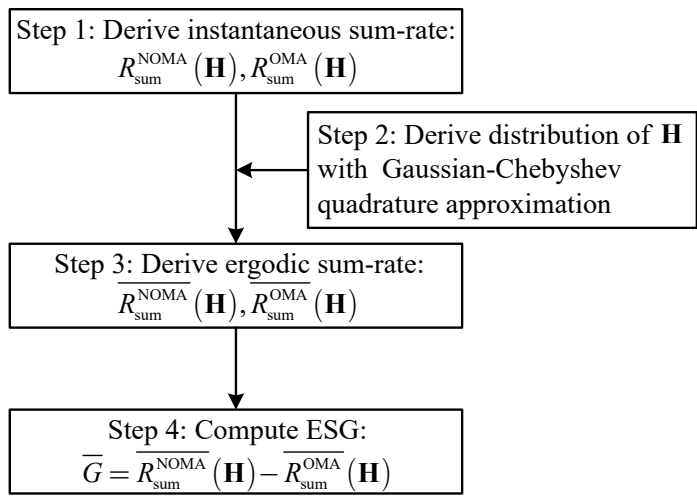

Fig. 2. A flow chart summarizes the adopted analysis procedure in this paper.

To avoid tedious comparison and to facilitate our performance analysis, we adopt a random user grouping strategy for the OMA systems considered and a fixed SIC detection order for the NOMA systems investigated. In particular, we randomly select $M$ and $W$ users for each group on each frequency subband for the MIMO-OMA and $m$ MIMO-OMA systems, respectively. For NOMA systems, without loss of generality, we assume $\left\|\mathbf{h}_{1}\right\| \geq\left\|\mathbf{h}_{2}\right\|, \ldots, \geq\left\|\mathbf{h}_{K}\right\|$, that the users are indexed based on their channel gains, and the SIC/MMSE-SIC/MRCSIC detection order ${ }^{9}$ at the $\mathrm{BS}$ is $1,2, \ldots, K$. Additionally, to unveil insights about the performance gain of NOMA over OMA, we assume that there is no error propagation during SIC/MMSE-SIC/MRC-SIC detection at the BS.

Remark 4: We note that without proper transmission rate allocation, both NOMA and OMA suffer from severe outage events. In some existing works [39], [44], the authors assumed fixed power as well as rate allocation and analyzed the outage probability of NOMA due to the randomness of channel fading. However, in this work, we focus on the ergodic sum-rate analysis where the transmission rate is determined opportunistically according to the instantaneous achievable rate. In particular, based on the channel estimates, the BS assigns the transmission rate for each user according to the instantaneous achievable rate for both NOMA and OMA schemes in different scenarios. However, in practice, the estimated CSI might be outdated during the rate allocation, which leads to the CSI error. The discussions of the impact of CSI error on the performance gain of NOMA over OMA can be found in Remark 2

\section{The Analysis Procedure}

In the rest of this paper, e.g. Sections III, IV, and V, a systematic procedure is adopted to analyze the ESG of NOMA over OMA, via combining the specific features in different scenarios. For the ease of illustration, we have summarized the 4-Step analysis procedure as a flow chart shown in Fig. 2. Step 1, based on the adopted signal detection and resource allocation strategies for NOMA and OMA in Table [I] we formulate the instantaneous sum-rate as a function of the

\footnotetext{
${ }^{9}$ Note that, in general, the adopted detection order is not the optimal SIC detection order for maximizing the achievable sum-rate of the considered MIMO-NOMA and $m$ MIMO-NOMA systems.
}

instantaneous channels of all the users, i.e., $R_{\mathrm{sum}}^{\mathrm{NOMA}}(\mathbf{H})$ and $R_{\text {sum }}^{\mathrm{OMA}}(\mathbf{H})$ with $\mathbf{H}=\left[\mathbf{h}_{1}, \ldots, \mathbf{h}_{K}\right]$. Then in Step 2, we derive the channel distributions in different scenarios. Note that an accurate polynomial approximation method, i.e., the GaussianChebyshev quadrature approximation [76], is employed to approximate the multi-dimensional integral for deriving the channel distribution ${ }^{10}$ Then, the third step is dedicated to derive the ergodic sum-rate via averaging the corresponding instantaneous sum-rate w.r.t. channel distributions. Finally in Step 4, the ESG can be obtained by comparing the ergodic sum-rates of NOMA and OMA. We note that in each step, asymptotic analysis techniques have been applied to provide some interesting insights on the performance gain of NOMA over OMA.

\section{ESG OF SISO-NOMA OVER SISO-OMA}

In this section, we first derive the ergodic sum-rate of SISO-NOMA and SISO-OMA. Then, the asymptotic ESG of SISO-NOMA over SISO-OMA is discussed under different scenarios.

\section{A. Ergodic Sum-rate of SISO-NOMA and SISO-OMA}

When detecing the messages of user $k$, the interferences imposed by users $1,2, \ldots, k-1$ have been canceled in the SISO-NOMA system by SIC reception. Therefore, the instantaneous achievable data rate of user $k$ in the SISONOMA system considered is given by:

$$
R_{k}^{\mathrm{SISO}-\mathrm{NOMA}}=\ln \left(1+\frac{p_{k}\left|h_{k}\right|^{2}}{\sum_{i=k+1}^{K} p_{i}\left|h_{i}\right|^{2}+N_{0}}\right) .
$$

On the other hand, for the considered SISO-OMA system, user $k$ is allocated to a subband exclusively, thus there is no inter-user interference (IUI). As a result, the instantaneous achievable data rate of user $k$ in the SISO-OMA system considered is given by:

$$
R_{k}^{\mathrm{SISO}-\mathrm{OMA}}=f_{k} \ln \left(1+\frac{p_{k}\left|h_{k}\right|^{2}}{f_{k} N_{0}}\right)
$$

with $p_{k}$ and $f_{k}$ denoting the power allocation and frequency allocation of user $k$. Note that we consider a normalized frequency bandwidth for both the NOMA and OMA schemes in this paper, i.e., $\sum_{k=1}^{K} f_{k}=1$. Under the identical resource allocation strategy, i.e., for $p_{k}=\frac{P_{\max }}{K}$ and $f_{k}=1 / K$, we have the instantaneous sum-rate of SISO-NOMA and SISO-

\footnotetext{
${ }^{10}$ The error analysis of the adopted Gaussian-Chebyshev quadrature approximation is presented in the proof of Lemma 1 in Appendix A.
} 
OMA given by

$$
\begin{aligned}
R_{\mathrm{sum}}^{\mathrm{SISO}-\mathrm{NOMA}} & =\sum_{k=1}^{K} R_{k}^{\mathrm{SISO}-\mathrm{NOMA}} \\
& =\ln \left(1+\frac{P_{\max }}{K N_{0}} \sum_{k=1}^{K}\left|h_{k}\right|^{2}\right) \text { and } \\
R_{\mathrm{sum}}^{\mathrm{SISO}-\mathrm{OMA}} & =\sum_{k=1}^{K} R_{k}^{\mathrm{SISO}-\mathrm{OMA}} \\
& =\frac{1}{K} \sum_{k=1}^{K} \ln \left(1+\frac{P_{\max }}{N_{0}}\left|h_{k}\right|^{2}\right)
\end{aligned}
$$

respectively.

Given the instantaneous sum-rates in (6) and (7), firstly we have to investigate the channel gain distribution before embarking on the derivation of the corresponding ergodic sumrates, which is given in the following lemma.

Lemma 1: The cumulative distribution function (CDF) and probability density function (PDF) of $|h|^{2}$ can be approximated by 11

$$
\begin{aligned}
& F_{|h|^{2}}(x) \approx 1-\frac{1}{D+D_{0}} \sum_{n=1}^{N} \beta_{n} e^{-c_{n} x} \text { and } \\
& f_{|h|^{2}}(x) \approx \frac{1}{D+D_{0}} \sum_{n=1}^{N} \beta_{n} c_{n} e^{-c_{n} x}, x \geq 0
\end{aligned}
$$

respectively, where the parameters in (8) and (9) are:

$$
\begin{aligned}
& \beta_{n}=\frac{\pi}{N}\left|\sin \frac{2 n-1}{2 N} \pi\right|\left(\frac{D-D_{0}}{2} \cos \frac{2 n-1}{2 N} \pi+\frac{D+D_{0}}{2}\right) \text { and } \\
& c_{n}=1+\left(\frac{D-D_{0}}{2} \cos \frac{2 n-1}{2 N} \pi+\frac{D+D_{0}}{2}\right)^{\alpha}
\end{aligned}
$$

while $N$ denotes the number of terms adopted for integral approximation.

Proof: Please refer to Appendix A for the proof of Lemma 1

Based on (6), the ergodic sum-rate of the SISO-NOMA system considered is defined as:

$$
\begin{aligned}
\overline{R_{\mathrm{sum}}^{\mathrm{SISO}-\mathrm{NOMA}}} & =\mathrm{E}_{\mathbf{H}}\left\{R_{\mathrm{sum}}^{\mathrm{SISO}-\mathrm{NOMA}}\right\} \\
& =\mathrm{E}_{\mathbf{H}}\left\{\ln \left(1+\frac{P_{\max }}{K N_{0}} \sum_{k=1}^{K}\left|h_{k}\right|^{2}\right)\right\},
\end{aligned}
$$

where the expectation $\mathrm{E}_{\mathbf{H}}\{\cdot\}$ is averaged over both the largescale fading and small-scale fading in the overall channel matrix H. For a large number of users, i.e., $K \rightarrow \infty$, the sum of channel gains of all the users within the $\ln (\cdot)$ in $(11)$ becomes a deterministic value due to the strong law of large

\footnotetext{
${ }^{11}$ As mentioned before, we assumed that the channel gains of all the users are ordered as $\left|h_{1}\right| \geq\left|h_{2}\right|, \ldots, \geq\left|h_{K}\right|$ in Section II-C2 However, as shown in (6), the system sum-rate for the considered SISO-NOMA system is actually independent of the SIC detection order. Therefore, we can safely assume that all the users have i.i.d. channel distribution, which does not affect the performance analysis results. In the sequel of this paper, the subscript $k$ is dropped without loss of generality.
}

numbers, i.e., $\lim _{K \rightarrow \infty} \frac{1}{K} \sum_{k=1}^{K}\left|h_{k}\right|^{2}=\overline{|h|^{2}}$, where $\overline{|h|^{2}}$ denotes the average channel power gain and it is given by

$$
\overline{|h|^{2}}=\int_{0}^{\infty} x f_{|h|^{2}}(x) d x \approx \frac{1}{D+D_{0}} \sum_{n=1}^{N} \frac{\beta_{n}}{c_{n}} .
$$

Therefore, the asymptotic ergodic sum-rate of the SISONOMA system considered is given by

$$
\begin{aligned}
& \lim _{K \rightarrow \infty} \overline{R_{\text {sum }}^{\mathrm{SISO}-\mathrm{NOMA}}} \stackrel{(a)}{=} \mathrm{E}_{\mathbf{H}}\left\{\lim _{K \rightarrow \infty} R_{\mathrm{sum}}^{\mathrm{SISO}-\mathrm{NOMA}}\right\} \\
& =\ln \left(1+\frac{P_{\max }}{N_{0}} \overline{|h|^{2}}\right) \\
& \approx \ln \left(1+\frac{P_{\max }}{\left(D+D_{0}\right) N_{0}} \sum_{n=1}^{N} \frac{\beta_{n}}{c_{n}}\right),
\end{aligned}
$$

where the equality $(a)$ is due to the bounded convergence theorem [77] and owing to the finite channel capacity. Note that for a finite number of users $K$, the asymptotic ergodic sum-rate in (13) serves as an upper bound for the actual ergodic sum-rate

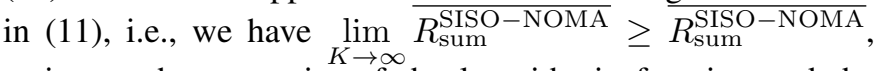
owing to the concavity of the logarithmic function and the Jensen's inequality. In the Section VII, we will show that the asymptotic analysis in (13) is also accurate for a finite value of $K$ and becomes exact for a sufficient large $K$.

Similarly, based on (7), we can obtain the ergodic sum-rate of the SISO-OMA system as follows:

$$
\begin{aligned}
\overline{R_{\mathrm{sum}}^{\mathrm{SISO}-\mathrm{OMA}}} & =\mathrm{E}_{\mathbf{H}}\left\{\frac{1}{K} \sum_{k=1}^{K} \ln \left(1+\frac{P_{\max }}{N_{0}}\left|h_{k}\right|^{2}\right)\right\} \\
& \stackrel{(a)}{=} \int_{0}^{\infty} \ln \left(1+\frac{P_{\max }}{N_{0}} x\right) f_{|h|^{2}}(x) d x \\
& =\frac{1}{\left(D+D_{0}\right)} \sum_{n=1}^{N} \beta_{n} e^{\frac{c_{n} N_{0}}{P_{\max }}} \mathcal{E}_{1}\left(\frac{c_{n} N_{0}}{P_{\max }}\right),
\end{aligned}
$$

where $\mathcal{E}_{l}(x)=\int_{1}^{\infty} \frac{e^{-x t}}{t^{l}} d t$ denotes the $l$-order exponential integral [56]. The equality $(a)$ in (14) is obtained since all the users have i.i.d. channel distributions. Note that in contrast

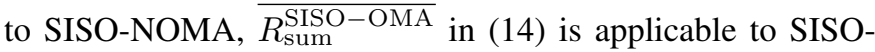
OMA supporting an arbitrary number of users.

\section{B. ESG in Single-antenna Systems}

Comparing (13) and (14), the asymptotic ESG of SISONOMA over SISO-OMA with $K \rightarrow \infty$ can be expressed as follows:

$$
\begin{aligned}
\lim _{K \rightarrow \infty} \overline{G^{\mathrm{SISO}}} & =\lim _{K \rightarrow \infty} \overline{R_{\mathrm{sum}}^{\mathrm{SISO}-\mathrm{NOMA}}}-\overline{R_{\mathrm{sum}}^{\mathrm{SISO}-\mathrm{OMA}}} \\
& \approx \ln \left(1+\frac{P_{\max }}{\left(D+D_{0}\right) N_{0}} \sum_{n=1}^{N} \frac{\beta_{n}}{c_{n}}\right) \\
& -\frac{1}{\left(D+D_{0}\right)} \sum_{n=1}^{N} \beta_{n} e^{\frac{c_{n} N_{0}}{P_{\max }}} \mathcal{E}_{1}\left(\frac{c_{n} N_{0}}{P_{\max }}\right)
\end{aligned}
$$


Then, in the high-SNR regim ${ }^{12}$, we can approximate the asymptotic ESG in 15 by applying $\lim _{x \rightarrow 0} \mathcal{E}_{1}(x) \approx-\ln (x)-\gamma$ [56] as

$$
\lim _{K \rightarrow \infty, P_{\max } \rightarrow \infty} \overline{G^{\mathrm{SISO}}} \approx \vartheta\left(D, D_{0}\right)+\gamma,
$$

where $\vartheta\left(D, D_{0}\right)$ is given by

$$
\vartheta\left(D, D_{0}\right)=\ln \left(\frac{\sum_{n=1}^{N}\left(\frac{1}{c_{n}}\right) \frac{\beta_{n}}{\left(D+D_{0}\right)}}{\prod_{n=1}^{N}\left(\frac{1}{c_{n}}\right)^{\frac{\beta_{n}}{\left(D+D_{0}\right)}}}\right)
$$

and $\gamma=0.57721$ is the Euler-Mascheroni constant [56]. Based on the weighted arithmetic and geometric means (AMGM) inequality [78], we can observe that $\vartheta\left(D, D_{0}\right) \geq 0$. This implies that $\lim _{K \rightarrow \infty, P_{\max } \rightarrow \infty} \overline{G^{\mathrm{SISO}}}>0$ and SISO-NOMA provides a higher asymptotic ergodic sum-rate than SISOOMA in the system considered.

To further simplify the expression of ESG, we consider path loss exponents $\alpha$ in the range of $\alpha \in[3,6]$ in $(10)$, which usually holds in urban environments [58]. As a result, $c_{n} \gg 1$. Hence, $\vartheta\left(D, D_{0}\right)$ in (17) can be further simplified as follows:

$$
\begin{aligned}
\vartheta\left(D, D_{0}\right) & \approx \vartheta(\eta)= \\
& \ln \left(\frac{\frac{\pi}{N(1+\eta)} \sum_{n=1}^{N}\left[\lambda_{n}(\eta)\right]^{1-\alpha}\left|\sin \frac{2 n-1}{2 N} \pi\right|}{\prod_{n=1}^{N}\left[\lambda_{n}(\eta)\right]^{-\frac{\alpha \pi \lambda_{n}(\eta)}{N(1+\eta)}\left|\sin \frac{2 n-1}{2 N} \pi\right|}}\right),
\end{aligned}
$$

where $\lambda_{n}(\eta)=\left(\frac{\eta-1}{2} \cos \left(\frac{2 n-1}{2 N} \pi\right)+\frac{\eta+1}{2}\right) \in[1, \eta)$. The normalized cell size of $\eta=\frac{D}{D_{0}} \geq 1$ is the ratio between the outer radius $D$ and the inner radius $D_{0}$, which also serves as a metric of the path loss discrepancy.

We can see that the asymptotic ESG of SISO-NOMA over SISO-OMA in 16 is composed of two components, i.e., $\vartheta\left(D, D_{0}\right)$ and $\gamma$. As observed in (18), the former component of $\vartheta\left(D, D_{0}\right) \approx \vartheta(\eta)$ only depends on the normalized cell size of $\eta=\frac{D}{D_{0}}$ instead of the absolute values of $D$ and $D_{0}$. In fact, it can characterize the large-scale near-far gain attained by NOMA via exploiting the discrepancy in distances among NOMA users. Interestingly, for the extreme case that all the users are randomly deployed on a circle, i.e., $D=D_{0}$, we have $\eta=1, \lambda_{n}(\eta)=1$, and $\vartheta(\eta)=0$. In other words, the large-scale near-far gain disappears, when all the users are of identical distance away from the BS. With the aid of $\vartheta(\eta)=0$, we can observe in 16 that the performance gain achieved by NOMA is a constant value of $\gamma=0.57721$ nat $/ \mathrm{s} / \mathrm{Hz}$. Since all the users are set to have the same distance when $D=D_{0}$, the minimum asymptotic ESG $\gamma$ arising from the small-scale Rayleigh fading is named as the small-scale fading gain in this paper. In fact, in the asymptotic case associated with $K \rightarrow \infty$ and $P_{\max } \rightarrow \infty$, SISO-NOMA provides at least $\gamma=0.57721 \mathrm{nat} / \mathrm{s} / \mathrm{Hz}$ spectral efficiency

\footnotetext{
${ }^{12}$ Under the sum-power constraint, the system SNR directly depends on the total system power budget $P_{\max }$, and thus the system SNR and $P_{\max }$ are interchangeable in this paper. Note that in this paper, we assume a large system SNR, as defined in (71), rather than a large individual SNR for each user for analyzing the ergodic sum-rate.
}

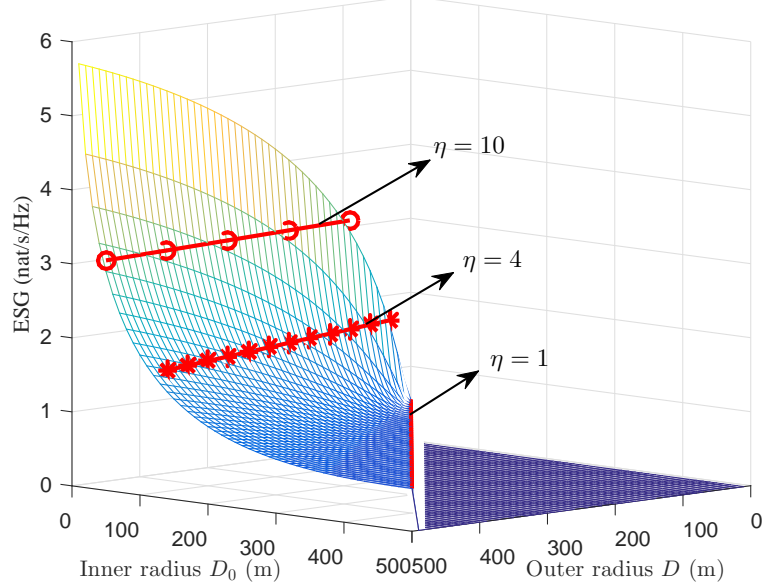

Fig. 3. The asymptotic ESG in 16 under equal resource allocation versus $D$ and $D_{0}$ with $K \rightarrow \infty$ and $P_{\max } \rightarrow \infty$.

gain over SISO-OMA for an arbitrary cell size in Rayleigh fading channels. Additionally, we can see that the ESG of SISO-NOMA over SISO-OMA is saturated in the high-SNR regime. This is because the instantaneous system sum-rates of both the SISO-NOMA system in (6) and the SISO-OMA system in (7) increase logarithmically with $P_{\max } \rightarrow \infty$.

To visualize the large-scale near-far gain, we illustrate the asymptotic ESG in (16) versus $D$ and $D_{0}$ in Fig. 3 We can observe that when $\eta=1$, the large-scale near-far gain disappears and the asymptotic ESG is bounded from below by its minimum value of $\gamma=0.57721 \mathrm{nat} / \mathrm{s} / \mathrm{Hz}$ due to the small-scale fading gain. Additionally, for different values of $D$ and $D_{0}$ but with a fixed $\eta=\frac{D}{D_{0}}$, SISO-NOMA offers the same ESG compared to SISO-OMA. This is because as predicted in (18), the large-scale near-far gain only depends on the normalized cell size $\eta$. More importantly, we can observe that the large-scale near-far gain increases with the normalized cell size $\eta$. In fact, for a larger normalized cell size $\eta$, the heterogeneity in the large-scale fading among users becomes higher and SISO-NOMA attains a higher near-far gain, hence improving the sum-rate performance.

Remark 5: Note that it has been analytically shown in [62] that two users with a large distance difference (or equivalently channel gain difference) are preferred to be paired. This is consistent with our conclusion in this paper, where a larger normalized cell size $\eta$ enables a higher ESG of NOMA over OMA. However, [62] only considered a pair of two NOMA users. In this paper, we analytically obtain the ESG of NOMA over OMA for a more general NOMA system supporting a large number of UL users. More importantly, we identify two kinds of gains in the ESG derived and reveal their different behaviours.

Remark 6: In this paper, we focus on the case of a large number of users and perfect SIC to facilitate the analytical study. In fact, the adopted system model with $K \rightarrow \infty$ is suitable for the application scenario of narrow band massive internet-of-thing (IoT) communications, where a large number of IoT devices intend to communicate with a common receiver 
through the same resource block [79], [80]. Although the proposed analysis is based on asymptotic performance, it can also be used to predict the performance of a finite value of $K$. As shown in Fig. 5 of Section VII] for a finite value of $K$, such as $K=128$ and $K=256$, the simulation results are close to the corresponding asymptotic analytical results. We note that a large finite NOMA group size may incur a high detection complexity and delay for the SIC implemented at the BS in the uplink of NOMA systems, although both only linearly increase with $K$ [69]. In addition, the asymptotic analytical results obtained in this treatise can be regarded as an upper bound of the performance gain of NOMA over OMA in practical implementations where the detection complexity and delay need to be considered. Moreover, the focus of asymptotic regime is a commonly adopted approach [39], [60], [81], [82] which can shed light on this complex problem for obtaining insightful yet tractable results.

On the other hand, although SIC detection suffers from the inevitable error propagation in practice, we assume the perfect SIC to facilitate our performance analysis. This is because modeling the error propagation during SIC detection is nontrivial from the achievable data rate perspective. It is true that there are some works in the literature considering the impact of imperfect SIC to the system performance, e.g. the performance analysis on coverage probability with considering the imperfect SIC for uplink NOMA can be found in [83]. Although we can follow a similar approach as [83] to analyze the impact of imperfect SIC on ESG, the results are generally intractable due to the involved $K$-dimensional integrations for calculating the system outage probability. As a first attempt to investigate the ESG, we would like to focus on the case of perfect SIC as fundamental system design insights can be unveiled. The impact of the SIC imperfection on the performance gain of NOMA over OMA will be investigated in Section VII via simulations.

\section{ESG OF MIMO-NOMA OVER MIMO-OMA}

In this section, the ergodic sum-rates of MIMO-NOMA and MIMO-OMA associated with FDMA-ZF as well as FDMA-MRC are firstly analyzed. Then, the asymptotic ESGs of MIMO-NOMA over MIMO-OMA with FDMA-ZF and FDMA-MRC detection are investigated.

\section{A. Ergodic Sum-rate of MIMO-NOMA with MMSE-SIC}

Let us consider that an $M$-antenna BS serves $K$ singleantenna non-orthogonal users relying on MIMO-NOMA. The BS employs MMSE-SIC detection for retrieving the messages of all the users. The instantaneous achievable data rate of user $k$ in the MIMO-NOMA system relying on MMSE-SIC detection ${ }^{13}$ is given by [27]:

$$
\begin{aligned}
R_{k}^{\mathrm{MIMO}-\mathrm{NOMA}} & =\ln \left|\mathbf{I}_{M}+\frac{1}{N_{0}} \sum_{i=k}^{K} p_{i} \mathbf{h}_{i} \mathbf{h}_{i}^{\mathrm{H}}\right| \\
& -\ln \left|\mathbf{I}_{M}+\frac{1}{N_{0}} \sum_{i=k+1}^{K} p_{i} \mathbf{h}_{i} \mathbf{h}_{i}^{\mathrm{H}}\right| .
\end{aligned}
$$

As a result, the instantaneous sum-rate of MIMO-NOMA is obtained as

$$
\begin{aligned}
R_{\mathrm{sum}}^{\mathrm{MIMO}-\mathrm{NOMA}} & =\sum_{k=1}^{K} R_{k}^{\mathrm{MIMO}-\mathrm{NOMA}} \\
& =\ln \left|\mathbf{I}_{M}+\frac{1}{N_{0}} \sum_{k=1}^{K} p_{k} \mathbf{h}_{k} \mathbf{h}_{k}^{\mathrm{H}}\right| .
\end{aligned}
$$

In fact, MMSE-SIC is capacity-achieving [27] and 20] is the channel capacity for a given instantaneous channel matrix $\mathbf{H}$ [84]. In general, it is a challenge to obtain a closed-form expression for the instantaneous channel capacity above due to the determinant of the summation of matrices in 20 . To provide some insights, in the following theorem, we consider an asymptotically tight upper bound for the achievable sumrate in 20] associated with $K \rightarrow \infty$.

Theorem 1: For the MIMO-NOMA system considered in (1) relying on MMSE-SIC detection, given any power allocation strategy $\mathbf{p}=\left[p_{1}, \ldots, p_{K}\right]$, the achievable sum-rate in 20] is upper bounded by

$$
R_{\text {sum }}^{\mathrm{MIMO}-\mathrm{NOMA}} \leq M \ln \left(1+\frac{1}{M N_{0}} \sum_{k=1}^{K} p_{k}\left\|\mathbf{h}_{k}\right\|^{2}\right) .
$$

This upper bound is asymptotically tight, when $K \rightarrow \infty$, i.e.,

$$
\begin{aligned}
& \lim _{K \rightarrow \infty} R_{\mathrm{sum}}^{\mathrm{MIMO}-\mathrm{NOMA}} \\
& =\lim _{K \rightarrow \infty} M \ln \left(1+\frac{1}{M N_{0}} \sum_{k=1}^{K} p_{k}\left\|\mathbf{h}_{k}\right\|^{2}\right) .
\end{aligned}
$$

Proof: Please refer to Appendix B for the proof of Theorem 1

Now, given the instantaneous achievable sum-rate obtained in 22, we proceed to calculate the ergodic sum-rate. Before that, we derive the distribution of $\|\mathbf{h}\|^{2}$ in the following lemma.

Lemma 2: The CDF and PDF of $\|\mathbf{h}\|^{2}$ can be written as

$$
\begin{aligned}
F_{\|\mathbf{h}\|^{2}}(x) & \approx 1-\frac{1}{D+D_{0}} \sum_{n=1}^{N} \frac{\beta_{n} \gamma_{L}\left(M, c_{n} x\right)}{\Gamma(M)} \text { and } \\
f_{\|\mathbf{h}\|^{2}}(x) & \approx \frac{1}{D+D_{0}} \sum_{n=1}^{N} \beta_{n} \operatorname{Gamma}\left(M, c_{n}, x\right), x \geq 0,
\end{aligned}
$$

\footnotetext{
${ }^{13}$ The derivation of individual rates in 19 ) for MMSE-SIC detection of MIMO-NOMA is based on the matrix inversion lemma:$$
\log \left|\mathbf{A}+\mathbf{h h}^{\mathrm{H}}\right|-\log |\mathbf{A}|=\log \left|1+\mathbf{h}^{\mathrm{H}} \mathbf{A}^{-1} \mathbf{h}\right| .
$$

Interested readers are referred to [27] for a detailed derivation.

${ }^{14}$ Similar to 8 and 9 , we can safely assume that all the users have i.i.d. channel distribution within the cell and drop the subscript $k$ in 89 , since the system sum-rate in 20 is independent of the MMSE-SIC detection order
} 27. 
respectively, where $\beta_{n}$ and $c_{n}$ are given in (10), $\operatorname{Gamma}(M, \lambda, x)=\frac{\lambda^{M} x^{M-1} e^{-\lambda x}}{\Gamma(M)}$ denotes the PDF of a random variable obeying a Gamma distribution, $\Gamma(M)$ denotes the Gamma function, and $\gamma_{L}\left(M,\left(1+d^{\alpha}\right) x\right)$ denotes the lower incomplete Gamma function.

Proof: Please refer to Appendix C for the proof of Lemma 2.

According to 22, given the equal resource allocation strategy, i.e., $p_{k}=\frac{P_{\max }}{K}$, the asymptotic ergodic sum-rate of MIMO-NOMA associated with $K \rightarrow \infty$ can be obtained as follows:

$$
\begin{aligned}
\lim _{K \rightarrow \infty} \overline{R_{\mathrm{sum}}^{\mathrm{MIMO}-\mathrm{NOMA}}} & =\lim _{K \rightarrow \infty} \mathrm{E}_{\mathbf{H}}\left\{R_{\mathrm{sum}}^{\mathrm{MIMO}-\mathrm{NOMA}}\right\} \\
& =M \ln \left(1+\frac{P_{\max }}{M N_{0}} \overline{\|\mathbf{h}\|^{2}}\right) \\
& \approx M \ln \left(1+\frac{P_{\max }}{\left(D+D_{0}\right) N_{0}} \sum_{n=1}^{N} \frac{\beta_{n}}{c_{n}}\right),
\end{aligned}
$$

where $\overline{\|\mathbf{h}\|^{2}}$ denotes the average channel gain, which is given by

$$
\overline{\|\mathbf{h}\|^{2}}=\int_{0}^{\infty} x f_{\|\mathbf{h}\|^{2}}(x) d x \approx \frac{M}{D+D_{0}} \sum_{n=1}^{N} \frac{\beta_{n}}{c_{n}} .
$$

Remark 7: Comparing (13) and (24), we can observe that for a sufficiently large number of users, the considered MIMONOMA system is asymptotically equivalent to a SISO-NOMA system with $M$-fold increases in DoF and an equivalent average channel gain of $\|\mathbf{h}\|^{2}$ in each DoF. Intuitively, when the number of UL receiver antennas at the BS, $M$, is much smaller than the number of users, $K \rightarrow \infty$, which corresponds to the extreme asymmetric case of MIMO-NOMA, the multiantenna BS behaves asymptotically in the same way as a single-antenna BS. Additionally, when $K \gg M$, due to the diverse channel directions of all the users, the received signals fully span the $M$-dimensional signal space [60]. Therefore, MIMO-NOMA using MMSE-SIC reception can fully exploit the system's spatial DoF, $M$, and its performance can be approximated by that of a SISO-NOMA system with $M$-fold DoF.

\section{B. Ergodic Sum-rate of MIMO-OMA with FDMA-ZF}

Upon installing more UL receiver antennas at the BS, ZF can be employed for MUD and the MIMO-OMA system using FDMA-ZF can accommodate $M$ users on each frequency subband. As mentioned before, we adopt a random user grouping strategy for the MIMO-OMA system using FDMAZF detection, where we randomly select $M$ users as a group and denote the composite channel matrix of the $g$-th group by $\mathbf{H}_{g}=\left[\mathbf{h}_{(g-1) M+1}, \mathbf{h}_{(g-1) M+2}, \ldots, \mathbf{h}_{g M}\right] \in \mathbb{C}^{M \times M}$. Then, the instantaneous achievable data rate of user $k$ in the MIMOOMA system is given by

$$
R_{k, \mathrm{FDMA}-\mathrm{ZF}}^{\mathrm{MIMO}-\mathrm{OMA}}=f_{g} \ln \left(1+\frac{p_{k}\left|\mathbf{w}_{g, k}^{\mathrm{H}} \mathbf{h}_{k}\right|^{2}}{f_{g} N_{0}}\right),
$$

where $f_{g}$ denotes the normalized frequency allocation for the $g$-th group. The vector $\mathbf{w}_{g, k} \in \mathbb{C}^{M \times 1}$ denotes the normalized ZF detection vector for user $k$ with $\left\|\mathbf{w}_{g, k}\right\|^{2}=1$, which is obtained based on the pseudoinverse of the composite channel matrix $\mathbf{H}_{g}$ in the $g$-th user group [27].

Given the equal resource allocation strategy, i.e., $p_{k}=\frac{P_{\max }}{K}$ and $f_{g}=1 / G=\frac{M}{K}$, the instantaneous sum-rate of MIMOOMA using FDMA-ZF can be formulated as:

$$
\begin{aligned}
R_{\text {sum,FDMA-ZF }}^{\mathrm{MIMO}} & =\sum_{k=1}^{K} R_{k, \mathrm{FDMA}-\mathrm{ZF}}^{\mathrm{MIMO-OMA}} \\
& =\frac{M}{K} \sum_{k=1}^{K} \ln \left(1+\frac{P_{\max }}{M N_{0}}\left|\mathbf{w}_{g, k}^{\mathrm{H}} \mathbf{h}_{k}\right|^{2}\right) .
\end{aligned}
$$

Since $\left\|\mathbf{w}_{g, k}\right\|^{2}=1$ and $\mathbf{g}_{k} \sim \mathcal{C N}\left(\mathbf{0}, \mathbf{I}_{M}\right)$, we have $\mathbf{w}_{g, k}^{\mathrm{H}} \mathbf{g}_{k} \sim$ $\mathcal{C N}(0,1)$ [27]. As a result, $\left|\mathbf{w}_{g, k}^{\mathrm{H}} \mathbf{h}_{k}\right|^{2}$ in 27] has an identical distribution with $|h|^{2}$, i.e., its CDF and PDF are given by (8) and 9, respectively. Therefore, the ergodic sum-rate of the MIMO-OMA system considered can be expressed as:

$$
\begin{aligned}
\overline{R_{\text {sum,FDMA-ZF }}^{\text {MIMO-OMA }}} & =\mathrm{E}_{\mathbf{H}}\left\{R_{\mathrm{sum}, \mathrm{FDMA}-\mathrm{MIF}}^{\mathrm{MIMO}-\mathrm{OMA}}\right\} \\
& =\int_{0}^{\infty} M \ln \left(1+\frac{P_{\max }}{M N_{0}} x\right) f_{|h|^{2}}(x) d x \\
& =\frac{M}{\left(D+D_{0}\right)} \sum_{n=1}^{N} \beta_{n} e^{\frac{c_{n} M N_{0}}{P_{\max }}} \mathcal{E}_{1}\left(\frac{c_{n} M N_{0}}{P_{\max }}\right) .
\end{aligned}
$$

\section{Ergodic Sum-rate of MIMO-OMA with FDMA-MRC}

The instantaneous achievable data rate of user $k$ in the MIMO-OMA system using the FDMA-MRC receiver is given by

$$
R_{k, \text { FDMA-MRC }}^{\mathrm{MIMO}-\mathrm{OMA}}=f_{k} \ln \left(1+\frac{p_{k}\left\|\mathbf{h}_{k}\right\|^{2}}{f_{k} N_{0}}\right) .
$$

Upon adopting the equal resource allocation strategy, i.e., $p_{k}=$ $\frac{P_{\max }}{K}$ and $f_{k}=1 / K$, the instantaneous sum-rate of MIMOOMA relying on FDMA-MRC is obtained by

$$
\begin{aligned}
R_{\mathrm{sum}, \mathrm{FDMA}-\mathrm{MRC}}^{\mathrm{MIMO}} & =\sum_{k=1}^{K} R_{k, \mathrm{FDMA}-\mathrm{MRC}}^{\mathrm{MIMO}-\mathrm{OMA}} \\
& =\frac{1}{K} \sum_{k=1}^{K} \ln \left(1+\frac{P_{\max }}{N_{0}}\left\|\mathbf{h}_{k}\right\|^{2}\right) .
\end{aligned}
$$

Averaging $R_{\text {sum,FDMA-MRC }}^{\mathrm{MIMO} \text { OMer }}$ the channel fading, we arrive at the ergodic sum-rate of MIMO-OMA using FDMAMRC as

$$
\begin{aligned}
\overline{R_{\text {sum,FDMA-MRC }}^{\mathrm{MIMOOOMA}}} & =\mathrm{E}_{\mathbf{H}}\left\{\frac{1}{K} \sum_{k=1}^{K} \ln \left(1+\frac{P_{\max }}{N_{0}}\left\|\mathbf{h}_{k}\right\|^{2}\right)\right\} \\
& =\int_{0}^{\infty} \ln \left(1+\frac{P_{\max }}{N_{0}} x\right) f_{\|\mathbf{h}\|^{2}}(x) d x \\
& =\frac{1}{\left(D+D_{0}\right)} \sum_{n=1}^{N} \beta_{n} T_{n}
\end{aligned}
$$


with $T_{n}$ given by

$$
\begin{aligned}
T_{n} & =\int_{0}^{\infty} \ln \left(1+\frac{P_{\max }}{N_{0}} x\right) \operatorname{Gamma}\left(M, c_{n}\right) d x \\
& \stackrel{(a)}{=} \int_{0}^{\infty} \ln (1+t) \operatorname{Gamma}\left(M, \frac{N_{0} c_{n}}{P_{\max }}\right) d t \\
& \stackrel{(b)}{=} \frac{\left(\frac{N_{0} c_{n}}{P_{\max }}\right)^{M}}{\Gamma(M)} \mathcal{G}_{2,3}^{3,1}\left(\begin{array}{c}
-M,-M+1 \\
-M,-M, 0
\end{array} \mid \frac{N_{0} c_{n}}{P_{\max }}\right),
\end{aligned}
$$

where $G_{p, q}^{m, n}(\cdot)$ denotes the Meijer G-function. The equality $(a)$ in 32 is obtained due to $t=\frac{P_{\max }}{N_{0}} x \sim$ Gamma $\left(M, \frac{N_{0} c_{n}}{P_{\max }}\right)$ and the equality $(b)$ in 32 is based on Equation (3) in [85]. Now, the ergodic sum-rate of MIMOOMA using FDMA-MRC can be written as Equation (33) on the top of next page.

Note that, the ergodic sum-rate in (33) is applicable to an arbitrary number of users $K$ and an arbitrary SNR, but it is too complicated to offer insights concerning the ESG of MIMONOMA over MIMO-OMA. Hence, based on (31), we derive the asymptotic ergodic sum-rate of MIMO-OMA with FDMA$\mathrm{MRC}$ in the low-SNR regime with $P_{\max } \rightarrow 0$ as follows:

$$
\begin{aligned}
\lim _{P_{\max } \rightarrow 0} \overline{R_{\text {sum,FDMA-MRC }}^{\text {MIMO-OMA }}} & =\frac{P_{\max } \overline{N_{0}}}{\|\mathbf{h}\|^{2}} \\
& =\frac{M P_{\max }}{N_{0}\left(D+D_{0}\right)} \sum_{n=1}^{N} \frac{\beta_{n}}{c_{n}} .
\end{aligned}
$$

On the other hand, in the high-SNR regime, based on (31), the asymptotic ergodic sum-rate of MIMO-OMA using FDMA$\mathrm{MRC}$ is given by

$$
\begin{aligned}
& \lim _{P_{\max } \rightarrow \infty} \overline{R_{\text {sum,FDMA-MRC }}^{\text {MIMO-OMA }}} \\
& =\ln \left(\frac{P_{\max }}{N_{0}}\right)+E_{\mathbf{h}}\left\{\ln \left(\|\mathbf{h}\|^{2}\right)\right\} .
\end{aligned}
$$

\section{ESG in Multi-antenna Systems}

By comparing 24) and (28), we have the asymptotic ESG of MIMO-NOMA over MIMO-OMA relying on FDMA-ZF given by Equation (36) on the top of next page. To unveil some insights, we consider the asymptotic ESG in the highSNR regime given by Equation (37) on the top of next page, where $\vartheta\left(D, D_{0}\right)$ denotes the large-scale near-far gain given in (17).

Remark 8: The identified two kinds of gains in ESG of the single-antenna system in (16) are also observed in the ESG of MIMO-NOMA over MIMO-OMA using FDMA-ZF in (37). Moreover, it can be observed that both the large-scale near-far gain $\vartheta\left(D, D_{0}\right)$ and the small-scale fading gain $\gamma$ are increased by $M$ times as indicated in 37). In fact, upon comparing (16) and (37), we have

$$
\begin{aligned}
& \lim _{K \rightarrow \infty, P_{\max } \rightarrow \infty} \overline{G_{\mathrm{FDMA}-\mathrm{ZF}}^{\mathrm{MIMO}}} \\
& =M \lim _{K \rightarrow \infty, P_{\max } \rightarrow \infty} \overline{G^{\mathrm{SISO}}}+M \ln (M),
\end{aligned}
$$

which implies that the asymptotic ESG of MIMO-NOMA over MIMO-OMA is $M$-times of that in single-antenna systems, when there are $M$ UL receiver antennas at the BS. In fact, for $K \rightarrow \infty$, the heterogeneity in channel directions of all the users allows the received signals to fully span across the $M$ dimensional signal space. Hence, MIMO-NOMA and MIMOOMA using FDMA-ZF can fully exploit the system's maximal spatial DoF $M$. Furthermore, we have an additional power gain of $\ln (M)$ in the second term in 38 . This is due to a factor of $\frac{1}{M}$ average power loss within each group for $\mathrm{ZF}$ projection to suppress the IUI in the MIMO-OMA system considered [27].

Comparing (24) and (33), the asymptotic ESG of MIMONOMA over MIMO-OMA with FDMA-MRC is obtained by Equation (39) on the top of next page. Then, based on (24) and (34), the asymptotic ESG of MIMO-NOMA over MIMOOMA with FDMA-MRC in the low-SNR regime is given by

$$
\lim _{K \rightarrow \infty, P_{\max } \rightarrow 0} \overline{R_{\text {sum,FDMA-MRC }}^{\text {MIMO-OMA }}}=0 .
$$

Not surprisingly, the performance gain of MIMO-NOMA over MIMO-OMA with FDMA-MRC vanishes in the low-SNR regime, which has been shown by simulations in existing works, [39] for example. In the high-SNR regime, the asymptotic ESG of MIMO-NOMA over MIMO-OMA with FDMAMRC can be obtained from 24 and 35 by

$$
\begin{aligned}
& \lim _{K \rightarrow \infty, P_{\max } \rightarrow \infty} \overline{G_{\text {FDMA-MRC }}^{\text {MIMO }}} \\
& \approx(M-1) \ln \left(\frac{P_{\max }}{\left(D+D_{0}\right) N_{0}} \sum_{n=1}^{N} \frac{\beta_{n}}{c_{n}}\right)-\ln (M)+\Delta
\end{aligned}
$$

where $\Delta=\ln \left(\mathrm{E}_{\mathbf{h}}\left\{\|\mathbf{h}\|^{2}\right\}\right)-\mathrm{E}_{\mathbf{h}}\left\{\ln \left(\|\mathbf{h}\|^{2}\right)\right\}$ denotes the gap between $\ln \left(\mathrm{E}_{\mathbf{h}}\left\{\|\mathbf{h}\|^{2}\right\}\right)$ and $\mathrm{E}_{\mathbf{h}}\left\{\ln \left(\|\mathbf{h}\|^{2}\right)\right\}$.

Although the closed-form ESG of MIMO-NOMA over MIMO-OMA is not available for the case of FDMA-MRC, the third term $\Delta$ in 41) is a constant for a given outer radius $D$ and inner radius $D_{0}$. Besides, it is expected that the first term in 41) dominates the ESG in the high-SNR regime. We can observe that the first term in 41) increases linearly with the system SNR in $\mathrm{dB}$ with a slope of $(M-1)$ in the high-SNR regime. In other words, there is an $(M-1)$-fold DoF gain [64] in the asymptotic ESG of MIMO-NOMA over MIMO-OMA using FDMA-MRC. In fact, MIMO-NOMA is essentially an $M \times K$ MIMO system on all resource blocks, i.e., time slots and frequency subbands, where the system maximal spatial DoF is limited by $M$ due to $M<K$. On the other hand, MIMO-OMA using the FDMA-MRC reception is always an $M \times 1$ MIMO system in each resource block, and thus it can only have a spatial DoF, which is one. As a result, an $(M-1)$ fold DoF gain can be achieved by MIMO-NOMA compared to MIMO-OMA using FDMA-MRC. However, MIMO-OMA is only capable of offering a power gain of $\ln (M)$ owing to the MRC detection utilized at the BS and thus the asymptotic ESG in (41) suffers from a power reduction by a factor of $\ln (M)$ in the second term.

\section{ESG OF $m$ MIMO-NOMA OVER $m$ MIMO-OMA}

In this section, we first derive the ergodic sum-rate of both $m$ MIMO-NOMA and $m$ MIMO-OMA and then discuss the asymptotic ESG of $m$ MIMO-NOMA over $m$ MIMO-OMA. 


$$
\overline{R_{\text {sum,FDMA-MRC }}^{\mathrm{MIMO}-\mathrm{OMA}}}=\frac{1}{\left(D+D_{0}\right)} \sum_{n=1}^{N} \beta_{n}\left(\frac{\left(\frac{N_{0} c_{n}}{P_{\max }}\right)^{M}}{\Gamma(M)} \mathcal{G}_{2,3}^{3,1}\left(\begin{array}{c|c}
-M,-M+1 \\
-M,-M, 0
\end{array} \mid \frac{N_{0} c_{n}}{P_{\max }}\right)\right)
$$

$$
\begin{aligned}
\lim _{K \rightarrow \infty} \overline{G_{\mathrm{FDMA}-\mathrm{MF}}^{\mathrm{MIMO}}} & =\lim _{K \rightarrow \infty} \overline{R_{\mathrm{Sum}}^{\mathrm{MIMO}-\mathrm{NOMA}}}-\overline{R_{\mathrm{sum}, \mathrm{FDMA}-\mathrm{MI}}^{\mathrm{MIMO}-\mathrm{OMA}}} \\
& \approx M \ln \left(1+\frac{P_{\max }}{\left(D+D_{0}\right) N_{0}} \sum_{n=1}^{N} \frac{\beta_{n}}{c_{n}}\right)-\frac{M}{\left(D+D_{0}\right)} \sum_{n=1}^{N} \beta_{n} e^{\frac{c_{n} M N_{0}}{P_{\max }} \mathcal{E}_{1}\left(\frac{c_{n} M N_{0}}{P_{\max }}\right)}
\end{aligned}
$$

$$
\lim _{K \rightarrow \infty, P_{\max } \rightarrow \infty} \overline{G_{\mathrm{FDMA}-\mathrm{ZF}}^{\mathrm{MIMO}}} \approx M \underbrace{\vartheta\left(D, D_{0}\right)}_{\text {large-scale near-far gain }}+M \ln (M)+M \underbrace{\gamma}_{\text {small-scale fading gain }}
$$

$$
\begin{aligned}
& \lim _{K \rightarrow \infty} \overline{G_{\mathrm{FDMA}-\mathrm{MRC}}^{\mathrm{MIMO}}}=\lim _{K \rightarrow \infty} \overline{R_{\mathrm{sum}}^{\mathrm{MIMO}-\mathrm{NOMA}}}-\overline{R_{\mathrm{sum}, \mathrm{MDMA}-\mathrm{MIMC}}^{\mathrm{MIOMA}}} \\
& \quad \approx M \ln \left(1+\frac{P_{\max }}{\left(D+D_{0}\right) N_{0}} \sum_{n=1}^{N} \frac{\beta_{n}}{c_{n}}\right)-\frac{1}{\left(D+D_{0}\right)} \sum_{n=1}^{N} \beta_{n}\left(\frac{\left(\frac{N_{0} c_{n}}{P_{\max }}\right)^{M}}{\Gamma(M)} \mathcal{G}_{2,3}^{3,1}\left(\begin{array}{c}
-M,-M+1 \\
-M,-M, 0
\end{array} \mid \begin{array}{c}
N_{0} c_{n} \\
P_{\max }
\end{array}\right)\right)
\end{aligned}
$$

\section{A. Ergodic Sum-rate with $D>D_{0}$}

Let us now apply NOMA to massive-MIMO systems, where a large-scale antenna array $(M \rightarrow \infty)$ is employed at the $\mathrm{BS}$ and all the $K$ users are equipped with a single antenna. A simple MRC-SIC receiver is adopted at the BS for data detection of $m \mathrm{MIMO}$-NOMA. The instantaneous achievable data rate of user $k$ and the sum-rate of the mMIMO-NOMA system using the MRC-SIC reception are given by

$$
R_{k}^{m \text { MIMO-NOMA }}=\ln \left(1+\frac{p_{k}\left\|\mathbf{h}_{k}\right\|^{2}}{\sum_{i=k+1}^{K} p_{i}\left\|\mathbf{h}_{i}\right\|^{2}\left|\mathbf{e}_{k}^{\mathrm{H}} \mathbf{e}_{i}\right|^{2}+N_{0}}\right) \text { and }
$$$$
R_{\mathrm{sum}}^{m \mathrm{MIMO}-\mathrm{NOMA}}=\sum_{k=1}^{K} R_{k}^{m \mathrm{MIMO}-\mathrm{NOMA}}
$$

respectively, where $\mathbf{e}_{k}=\frac{\mathbf{h}_{k}}{\left\|\mathbf{h}_{k}\right\|}$ denotes the channel direction of user $k$. For the massive-MIMO system associated with $D>$ $D_{0}$, the asymptotic ergodic sum-rate of $K \rightarrow \infty$ and $M \rightarrow \infty$ is given in the following theorem.

Theorem 2: For the $m \mathrm{MIMO}-\mathrm{NOMA}$ system considered in (1) in conjunction with $D>D_{0}$ and MRC-SIC detection at the BS, under the equal resource allocation strategy, i.e., $p_{k}=\frac{P_{\max }}{K}, \forall k$, the asymptotic ergodic sum-rate can be approximated by Equation (44) on the top of next page, where

$$
\begin{aligned}
\phi_{n} & =\frac{D-D_{0}}{2} \cos \frac{2 n-1}{2 N} \pi+\frac{D+D_{0}}{2}, \\
\psi_{k} & =\frac{P_{\max } M}{\sum_{i=k+1}^{K} P_{\max } I_{i}+K N_{0}}, \text { and } \\
I_{k} & =\mathrm{E}_{d_{k}}\left\{\frac{1}{1+d_{k}^{\alpha}}\right\} \\
& =\left(\begin{array}{c}
K \\
k
\end{array}\right) \frac{k}{D+D_{0}} \sum_{n=1}^{N} \frac{\beta_{n}}{c_{n}}\left(\frac{\phi_{n}^{2}-D_{0}^{2}}{D^{2}-D_{0}^{2}}\right)^{k-1}\left(\frac{D^{2}-\phi_{n}^{2}}{D^{2}-D_{0}^{2}}\right)^{K-k} .
\end{aligned}
$$

Proof: Please refer to Appendix D for the proof of Theorem 2 .

For the $m$ MIMO-OMA system using the FDMA-MRC detection, we can allocate more than one user to each frequency subband due to the above-mentioned favorable propagation property [68]. In particular, upon allocating $W=\varsigma M$ users to each frequency subband with $\varsigma=\frac{W}{M} \ll 1$, the orthogonality among channel vectors of the $W$ users holds fairly well, hence the IUI becomes negligible ${ }^{15}$ Therefore, a random user grouping strategy is adopted, where we randomly select $W=\varsigma M$ users as a group and there are $G=\frac{K}{W}$ group$\$ 16$ separated using orthogonal frequency subbands. In each subband, low-complexity MRC detection can be employed for each individual user and thus the instantaneous achievable data

\footnotetext{
${ }^{15}$ We note that the favorable propagation property is not applicable to the considered $m$ MIMO-NOMA system with $\frac{M}{K}=\delta<1$. When detecting user $k$ at the BS, although the IUI power from an individual user is small, the aggregated IUI power from all remained users $k+1, k+2, \ldots, K$ is not negligible as $K=\frac{M}{\delta} \rightarrow \infty$.

${ }^{16}$ Without loss of generality, we consider that $K$ is an integer multiple of $G$ and $W$.
} 


$$
\begin{aligned}
\lim _{K \rightarrow \infty, M \rightarrow \infty} \overline{R_{\mathrm{sum}}^{m \text { MIMO-NOMA }}} & =\lim _{K \rightarrow \infty, M \rightarrow \infty} \mathrm{E}_{\mathbf{H}}\left\{R_{\mathrm{sum}}^{m \text { MIMO-NOMA }}\right\} \\
& \approx \lim _{K \rightarrow \infty, M \rightarrow \infty} \sum_{k=1}^{K}\left(\begin{array}{c}
K \\
k
\end{array}\right) \frac{k}{D+D_{0}} \sum_{n=1}^{N} \beta_{n} \ln \left(1+\frac{\psi_{k}}{c_{n}}\right)\left(\frac{\phi_{n}^{2}-D_{0}^{2}}{D^{2}-D_{0}^{2}}\right)^{k-1}\left(\frac{D^{2}-\phi_{n}^{2}}{D^{2}-D_{0}^{2}}\right)^{K-k}
\end{aligned}
$$

rate of user $k$ can be expressed by

$$
R_{k}^{m \text { MIMO-OMA }}=f_{g} \ln \left(1+\frac{p_{k}\left\|\mathbf{h}_{k}\right\|^{2}}{f_{g} N_{0}}\right),
$$

where $f_{g}$ denotes the normalized frequency allocation of the $g$-th group. Note that (46) serves as an upper bound of the instantaneous achievable data rate of user $k$ in the mMIMOOMA system, since we assumed it to be IUI-free. Then, under the equal resource allocation strategy, i.e., $p_{k}=\frac{P_{\max }}{K}$ and $f_{g}=1 / G=\frac{W}{K}=\delta \varsigma$, we have the asymptotic ergodic sumrate of the $m$ MIMO-OMA system associated with $D>D_{0}$ given by Equation (47) on the top of next page, where $\phi_{n}$ is given in 45 and $\xi=\frac{P_{\max }}{\varsigma N_{0}}$.

\section{B. Ergodic Sum-rate with $D=D_{0}$}

We note that the analytical results in (44) and (47) are only applicable to the system having $D>D_{0}$. The asymptotic ergodic sum-rate of the $m$ MIMO-NOMA system with $D=D_{0}$ can be expressed using the following theorem.

Theorem 3: With $D=D_{0}$ and the equal resource allocation strategy, i.e., $p_{k}=\frac{P_{\max }}{K}$ and $f_{g}=1 / G=\frac{W}{K}=\delta \varsigma$, the asymptotic ergodic sum-rates of the $m$ MIMO-NOMA system and of the $m$ MIMO-OMA system can be formulated by Equations (48) and (49) on the top of next page, respectively, where $\delta=\frac{M}{K}$ and $\varsigma=\frac{W}{M}$ are constants and $\varpi=\frac{P_{\max }}{\left(1+D_{0}^{\alpha}\right) N_{0}}$ denotes the total average received SNR of all the users.

Proof: Please refer to Appendix E for the proof of Theorem 3 .

\section{ESG in Massive-antenna Systems}

Based on (44) and (47), when $D>D_{0}$, the asymptotic ESG of $m$ MIMO-NOMA over $m$ MIMO-OMA associated with $K \rightarrow \infty$ and $M \rightarrow \infty$ can be expressed as Equation (50) on the top of next page. However, the expression in (50) is too complicated and does not provide immediate insights. Hence, we focus on the case of $D=D_{0}$ to unveil some important and plausible insights on the ESG of NOMA over OMA in the massive-MIMO system. The simulation results of Section VII will show that the insights obtained from the case of $D=D_{0}$ are also applicable to the general scenario of $D>D_{0}$.

Comparing (48) and (49), when $D=D_{0}$, we have the asymptotic ESG of $m$ MIMO-NOMA over mMIMO-OMA for $K \rightarrow \infty$ and $M \rightarrow \infty$ given by Equation 51 on the top of next page. In the low-SNR regime, we can observe that $\lim _{K \rightarrow \infty, M \rightarrow \infty, P_{\max } \rightarrow 0} \overline{G_{D=D_{0}}^{m \mathrm{MIMO}}} \rightarrow 0$. This implies that no gain can be achieved by NOMA in the low-SNR regime, which is consistent with (40). By contrast, in the high-SNR regime, we have asymptotic ESG given by Equations (52) and 53 on the top of next page, where $\zeta=[\ln (1+\varpi \delta+\varpi)(1+\delta)-\ln (1+\varpi \delta) \delta-\ln (1+\varpi)]$ represents the extra ergodic sum-rate gain upon supporting an extra user by the mMIMO-NOMA system considered. Explicitly, for $K \rightarrow \infty, M \rightarrow \infty$, and $P_{\max } \rightarrow \infty$, the resultant extra benefit $\zeta$ is jointly determined by the average received sum SNR $\varpi$ and the fixed ratio $\delta$. Observe in (52) and (53) that given the average received sum SNR $\varpi$ and the fixed ratios $\delta$ and $\varsigma$, the asymptotic ESG scales linearly with both the number of UL receiver antennas at the BS, $M$ and the number of users, $K$, respectively. In other words, the asymptotic ESG per user and the asymptotic ESG per antenna of $m$ MIMO-NOMA over $m$ MIMO-OMA are constant and they are given by

$$
\begin{aligned}
\lim _{K \rightarrow \infty, M \rightarrow \infty, P_{\max } \rightarrow \infty} & \frac{\overline{G_{D=D_{0}}^{m \mathrm{MIMO}}}}{K}=\zeta-\delta \varsigma \ln \left(1+\frac{\varpi}{\varsigma}\right) \text { and } \\
\lim _{K \rightarrow \infty, M \rightarrow \infty, P_{\max } \rightarrow \infty} & \frac{\overline{G_{D=D_{0}}^{m \mathrm{MIMO}}}}{M}=\frac{\zeta}{\delta}-\varsigma \ln \left(1+\frac{\varpi}{\varsigma}\right),
\end{aligned}
$$

respectively. We can explain this observation from the spatial DoF perspective, since it determines the pre-log factor for the ergodic sum-rate of both $m$ MIMO-NOMA and $m$ MIMOOMA and thus also determines the pre-log factor of the corresponding ESG. In particular, the $m$ MIMO-NOMA system considered is basically an $(M \times K)$ MIMO system associated with $M<K$, since all the $K$ users transmit their signals simultaneously in the same frequency band. When scaling up the $m$ MIMO-NOMA system while maintaining a fixed ratio $\delta=\frac{M}{K}$, the system's spatial DoF increases linearly both with $M$ and $K$. On the other hand, the spatial DoF of the $m$ MIMOOMA system is limited by the group size $W$, since it is always an $(M \times W)$ MIMO system associated with $W \ll M$ in each time slot and frequency subband. Therefore, the system's spatial DoF increases linearly with both $W$, and $M$, as well as $K$, when scaling up the $m$ MIMO-OMA system under fixed ratios of $\delta=\frac{M}{K}$ and $\varsigma=\frac{W}{M}$. As a result, due to the linear increase of the spatial DoF with $M$ as well as $K$ for both the $m$ MIMO-NOMA and $m$ MIMO-OMA systems, the asymptotic ESG increases linearly with both $M$ and $K$. Note that in contrast to (41), there is no DoF gain, despite the fact that the asymptotic ESG scales linearly both with $M$ as well as $K$. This is because the extra benefit $\zeta$ does not increase linearly with the system's SNR in $\mathrm{dB}$. As a result, the asymptotic ESG of $m$ MIMO-NOMA over $m$ MIMO-OMA cannot increase linearly with the system's SNR in dB, which will be shown in Section VII. 


$$
\begin{aligned}
\lim _{M \rightarrow \infty} \overline{R_{\text {sum }}^{m M I M O-O M A}} & =\lim _{M \rightarrow \infty} \mathrm{E}_{\mathbf{H}}\left\{R_{\text {sum }}^{m \text { MIMO-OMA }}\right\} \\
& =\lim _{M \rightarrow \infty} \delta \varsigma \sum_{k=1}^{K}\left(\begin{array}{c}
K \\
k
\end{array}\right) \frac{k}{D+D_{0}} \sum_{n=1}^{N} \beta_{n} \ln \left(1+\frac{\xi}{c_{n}}\right)\left(\frac{\phi_{n}^{2}-D_{0}^{2}}{D^{2}-D_{0}^{2}}\right)^{k-1}\left(\frac{D^{2}-\phi_{n}^{2}}{D^{2}-D_{0}^{2}}\right)^{K-k}
\end{aligned}
$$

$$
\begin{aligned}
\lim _{K \rightarrow \infty, M \rightarrow \infty} \overline{R_{\text {sum }}^{m \mathrm{MIMO}-\mathrm{NOMA}}} & \approx \lim _{K \rightarrow \infty, M \rightarrow \infty} \frac{M}{\varpi \delta}[\ln (1+\varpi \delta+\varpi)(1+\varpi \delta+\varpi) \\
& -\ln (1+\varpi \delta)(1+\varpi \delta)-\ln (1+\varpi)(1+\varpi)] \\
\lim _{M \rightarrow \infty} \overline{R_{\text {sum }}^{m \mathrm{MIMO}-\text { OMA }}} & =\lim _{M \rightarrow \infty} \varsigma M \ln \left(1+\frac{\varpi}{\varsigma}\right)
\end{aligned}
$$

$$
\begin{aligned}
\lim _{K \rightarrow \infty, M \rightarrow \infty} \overline{G_{D>D_{0}}^{m \mathrm{MIMO}}} & \approx \lim _{K \rightarrow \infty, M \rightarrow \infty} \sum_{k=1}^{K}\left(\begin{array}{c}
K \\
k
\end{array}\right) \frac{k}{D+D_{0}} \sum_{n=1}^{N} \beta_{n}\left[\ln \left(1+\frac{\psi_{k}}{c_{n}}\right)-\delta \varsigma \ln \left(1+\frac{\xi}{c_{n}}\right)\right] \\
& \times\left(\frac{\phi_{n}^{2}-D_{0}^{2}}{D^{2}-D_{0}^{2}}\right)^{k-1}\left(\frac{D^{2}-\phi_{n}^{2}}{D^{2}-D_{0}^{2}}\right)^{K-k}
\end{aligned}
$$

$$
\begin{aligned}
\lim _{K \rightarrow \infty, M \rightarrow \infty} \overline{G_{D=D_{0}}^{m \mathrm{MIMO}}} & =\lim _{K \rightarrow \infty, M \rightarrow \infty} \overline{R_{\mathrm{sum}}^{m \mathrm{MIMO}-\mathrm{NOMA}}}-\overline{R_{\mathrm{sum}}^{m \mathrm{MIMO}-\mathrm{OMA}}} \\
& \approx \lim _{K \rightarrow \infty, M \rightarrow \infty} \frac{M}{\varpi \delta}[\ln (1+\varpi \delta+\varpi)(1+\varpi \delta+\varpi) \\
& -\ln (1+\varpi \delta)(1+\varpi \delta)-\ln (1+\varpi)(1+\varpi)]-\varsigma M \ln \left(1+\frac{\varpi}{\varsigma}\right)
\end{aligned}
$$

$$
\begin{aligned}
& \lim _{K \rightarrow \infty, M \rightarrow \infty, P_{\max } \rightarrow \infty} \overline{G_{D=D_{0}}^{m \mathrm{MIMO}}} \approx \lim _{K \rightarrow \infty, M \rightarrow \infty, P_{\max } \rightarrow \infty} \frac{M}{\delta} \zeta-\varsigma M \ln \left(1+\frac{\varpi}{\varsigma}\right) \\
& =\lim _{K \rightarrow \infty, M \rightarrow \infty, P_{\max } \rightarrow \infty} K \zeta-\delta \varsigma K \ln \left(1+\frac{\varpi}{\varsigma}\right)
\end{aligned}
$$

\section{ESG IN MULTI-CELl SyStemS}

In Section III, the performance gain of NOMA over OMA has been investigated in single-cell systems, since these analytical results are easily comprehensible and reveal directly plausible insights. Naturally, the performance gain of NOMA over OMA in single-cell systems serves as an upper bound on that of non-cooperative multi-cell systems, which can be approached by employing conservative frequency reuse strategy. In practice, cellular networks consist of multiple cells where the inter-cell interference (ICI) is inevitable [86]. Furthermore, the characteristics of the ICI for NOMA and OMA schemes are different. In particular, ICI is imposed by all the users in adjacent cells for NOMA schemes, while only a subset of users inflict ICI in OMA schemes, as an explicit benefit of orthogonal time or frequency allocation. As a result, NOMA systems face more severe ICI than that of OMA, hence it remains unclear, if applying NOMA is still beneficial in multi-cell systems. Therefore, in this section, we investigate the ESG of NOMA over OMA in multi-cell systems.

\section{A. Inter-cell Interference in NOMA and OMA Systems}

Consider a multi-cell system having multiple nonoverlapped adjacent cells with index $l=1, \ldots, L$, which are randomly deployed and surround the serving cell $l=0$, as shown in Fig. 4. We assume that the $L$ interfering cells have the same structure as the serving cell and they are uniformly distributed in the pair of concentric ring-shaped discs of Fig. 4 having the inner radius of $D$ and outer radius of $D_{1}$. Furthermore, we adopt the radical frequency reuse factor of 1, i.e., using the same frequency band for all cells to facilitate the performance analysis ${ }^{17}$ Again, we are assuming that in each cell there is a single $M$-antenna BS serving $K$ single-antenna users in the UL and thus there are $K L$ users imposing interference on the serving BS. Additionally,

\footnotetext{
${ }^{17}$ With a less-aggressive frequency reuse strategy in multi-cell systems, both NOMA and OMA schemes endure less ICI since only the adjacent cells using the same frequency band with the serving cell are taken into account. As a result, the performance analyses derived in this paper can be extended to the case with a lower frequency reuse ratio by simply decreasing number of adjacent cells $L$. Again, the resultant performance will then approach the performance upper-bound of the single-cell scenario.
} 


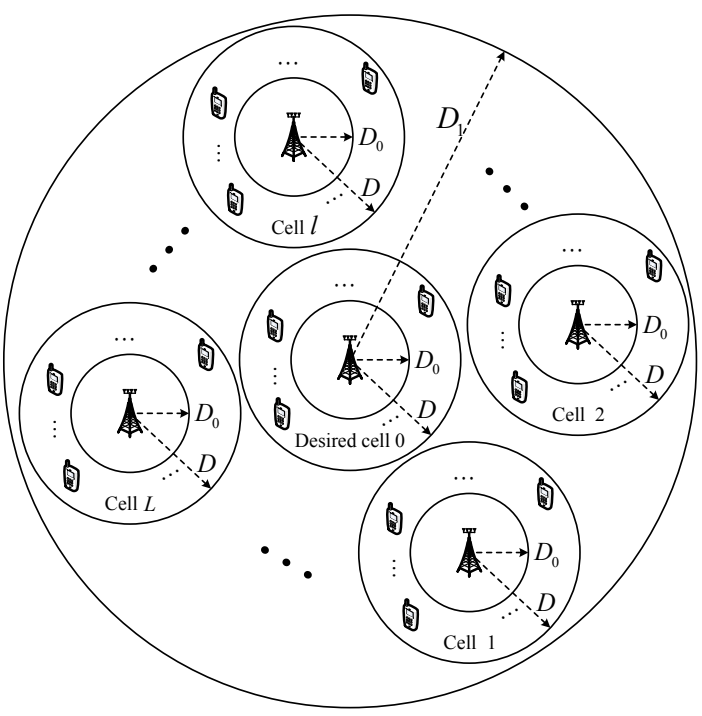

Fig. 4. The system model of the multi-cell uplink communication with one serving cell and $L$ adjacent cells.

to reduce both the system's overhead and its complexity, no cooperative multi-cell processing is included in our multi-cell system considered. In the following, we first investigate the resultant ICI distribution and then derive the total received ICI power contaminating over NOMA and OMA systems.

Given the normalized UL receive beamforming vector of user $k$ in the serving cell at the serving BS represented by $\mathbf{w}_{k} \in \mathbb{C}^{M \times 1}$ with $\left\|\mathbf{w}_{k}\right\|^{2}=1$, the effective ICI channel spanning from user $k^{\prime}$ in adjacent cell $l$ to the serving BS can be formulated as:

$$
h_{k^{\prime}, l}=\mathbf{w}_{k}^{\mathrm{H}} \mathbf{h}_{k^{\prime}, l}=\frac{\mathbf{w}_{k}^{\mathrm{H}} \mathbf{g}_{k^{\prime}, l}}{\sqrt{1+d_{k^{\prime}, l}^{\alpha}}},
$$

where $\mathbf{h}_{k^{\prime}, l}=\frac{\mathbf{g}_{k^{\prime}, l}}{\sqrt{1+d_{k^{\prime}, l}^{\alpha}}}$ denotes the channel vector from user $k^{\prime}$ in adjacent cell $l$ to the serving BS, $\mathrm{g}_{k^{\prime}, l} \in \mathbb{C}^{M \times 1}$ represents the Rayleigh fading coefficients, i.e., $\mathbf{g}_{k^{\prime}, l} \sim \mathcal{C N}\left(\mathbf{0}, \mathbf{I}_{M}\right)$, and $d_{k^{\prime}, l}$ denotes the distance between user $k^{\prime}$ in adjacent cell $l$ and the serving BS with the unit of meter. Similar to the single-cell system considered, we assume that the CSI of all the users within the serving cell is perfectly known at the serving BS. However, the ICI channel is unknown for the serving BS. Note that the receive beamformer $\mathbf{w}_{k}$ of the serving BS depends on the instantaneous channel vector of user $k, \mathbf{h}_{k}$, and/or on the multiple access interference structure $\left[\mathbf{h}_{1}, \ldots, \mathbf{h}_{k-1}, \mathbf{h}_{k+1}, \ldots, \mathbf{h}_{K}\right]$ in the serving cell. Therefore, the receive beamformer $\mathbf{w}_{k}$ of the serving cell is independent of the ICI channel $\mathbf{g}_{k^{\prime}, l}$. As a result, owing to $\left\|\mathbf{w}_{k}\right\|^{2}=1$, it can be readily observed that $\mathbf{w}_{k}^{\mathrm{H}} \mathbf{g}_{k^{\prime}, l}$ obeys the circularly symmetric complex Gaussian distribution conditioned on the given $\mathbf{w}_{k}$, i.e., we have $\left.\mathbf{w}_{k}^{\mathrm{H}} \mathbf{g}_{k^{\prime}, l}\right|_{\mathbf{w}_{k}} \sim \mathcal{C N}(0,1)$. However, since the resultant distribution $\mathcal{C N}(0,1)$ is independent of $\mathbf{w}_{k}$, we can safely drop the condition and directly apply $\mathbf{w}_{k}^{\mathrm{H}} \mathbf{g}_{k^{\prime}, l} \sim \mathcal{C N}(0,1)$. Now, based on 56, we can observe that the effective ICI channel $h_{k^{\prime}, l}$ is equivalent to a singleantenna Rayleigh fading channel associated with a distance of $d_{k^{\prime}, l}$, regardless of how many antennas are employed at the serving BS.

Given that each uplink user is equipped with a singleantenna, the transmission of each user is omnidirectional. Therefore, to facilitate the analysis of the ICI power, we assume that there is no gap between the adjacent cells and that the inner radius of each adjacent cell is zero, i.e., $D_{0}=0$. Hence, we can further assume that the ICI emanates from $K L$ users uniformly distributed within the ring-shaped disc having the inner radius of $D$ and outer radius of $D_{1}$. Similar to 8 ) and (9), the CDF and PDF of $\left|h_{k^{\prime}, l}\right|^{2}$ are given by

$$
\begin{aligned}
& F_{\left|h_{k^{\prime}, l}\right|^{2}}(x) \approx 1-\frac{1}{D+D_{1}} \sum_{n=1}^{N} \beta_{n}^{\prime} e^{-c_{n}^{\prime} x} \text { and } \\
& f_{\left|h_{k^{\prime}, l}\right|^{2}}(x) \approx \frac{1}{D+D_{1}} \sum_{n=1}^{N} \beta_{n}^{\prime} c_{n}^{\prime} e^{-c_{n}^{\prime} x}, x \geq 0, \forall k^{\prime}, l
\end{aligned}
$$

respectively, with parameters of

$$
\begin{aligned}
& \beta_{n}^{\prime}=\frac{\pi}{N}\left|\sin \frac{2 n-1}{2 N} \pi\right|\left(\frac{D_{1}-D}{2} \cos \frac{2 n-1}{2 N} \pi+\frac{D_{1}+D}{2}\right) \text { and } \\
& c_{n}^{\prime}=1+\left(\frac{D_{1}-D}{2} \cos \frac{2 n-1}{2 N} \pi+\frac{D_{1}+D}{2}\right)^{\alpha}
\end{aligned}
$$

Note that all the adjacent cell users have i.i.d. channel distributions since we ignore the adjacent cells' structure.

Due to the ICI encountered in unity-frequency-reuse multicell systems, the performance is determined by the signalto-interference-plus-noise ratio (SINR) instead of the SNR of single-cell systems. Assuming that the ICI is treated as AWGN by the detector, the system's SINR can be defined as follows:

$$
\mathrm{SINR}_{\mathrm{sum}}^{\text {multicell }}=\frac{P_{\max }}{I_{\text {inter }}+N_{0}} \overline{|h|^{2}},
$$

where $I_{\text {inter }}$ characterizes the ICI power in multi-cell systems and $P_{\max }$ denotes the same system power budget in each single cell.

To facilitate our performance analysis, we assume that the equal resource allocation strategy is adopted in all the adjacent cells, i.e., $p_{k^{\prime}, l}=\frac{P_{\max }}{K}, \forall k^{\prime}, l$. When invoking NOMA in a multi-cell system, the ICI power can be modeled as

$$
I_{\text {inter }}^{\text {NOMA }}=\sum_{l=1}^{L} \sum_{k^{\prime}=1}^{K} \frac{P_{\max }}{K}\left|h_{k^{\prime}, l}\right|^{2} .
$$

For $K L \rightarrow \infty, I_{\text {inter }}^{\text {NOMA }}$ becomes a deterministic value ${ }^{18}$ which can be approximated by

$$
\lim _{K L \rightarrow \infty} I_{\text {inter }}^{\mathrm{NOMA}} \approx L P_{\max } \overline{\left|h_{k^{\prime}, l}\right|^{2}} \approx \frac{L P_{\max }}{D+D_{1}} \sum_{n=1}^{N} \frac{\beta_{n}^{\prime}}{c_{n}^{\prime}} .
$$

As a result, the SINR of the multi-cell NOMA system considered is given by

$$
\mathrm{SINR}_{\mathrm{sum}, \mathrm{NOMA}}^{\text {multicell }}=\frac{P_{\max }}{\frac{L P_{\max }}{D+D_{1}} \sum_{n=1}^{N} \frac{\beta_{n}^{\prime}}{c_{n}^{\prime}}+N_{0}} \overline{|h|^{2}} .
$$

\footnotetext{
${ }^{18}$ Note that a spatially uniform distribution for the locations of both adjacent BSs as well as users has been assumed, which is a special case of Poisson point process [87.
} 
For OMA schemes, we assume that all the $K$ users in each cell are clustered into $G$ groups, with each group allocated to a frequency subband exclusively. Since only $\frac{1}{G}$ of users in each adjacent cell are simultaneously transmitting their signals in each frequency subband, the ICI power in a multi-cell OMA system can be expressed as:

$$
\lim _{K L \rightarrow \infty} I_{\text {inter }}^{\mathrm{OMA}}=\frac{1}{G} \lim _{K L \rightarrow \infty} I_{\text {inter }}^{\mathrm{NOMA}} \approx \frac{L P_{\max }}{G\left(D+D_{1}\right)} \sum_{n=1}^{N} \frac{\beta_{n}^{\prime}}{c_{n}^{\prime}} .
$$

The SINR of the multi-cell OMA system considered can be written as:

$$
\mathrm{SINR}_{\text {sum,OMA }}^{\text {multicell }}=\frac{P_{\max }}{\frac{L P_{\max }}{G\left(D+D_{1}\right)} \sum_{n=1}^{N} \frac{\beta_{n}^{\prime}}{c_{n}^{\prime}}+\frac{1}{G} N_{0}} \overline{|h|^{2}} .
$$

Note that we have $G=K$ for SISO-OMA and MIMO-OMA with FDMA-MRC, $G=\frac{K}{M}$ for MIMO-OMA with FDMA-ZF, and $G=\frac{K}{W}$ for $m$ MIMO-OMA with FDMA-MRC.

\section{B. ESG in Multi-cell Systems}

It can be observed that $I_{\mathrm{inter}}^{\mathrm{NOMA}}$ in 62 and $I_{\mathrm{inter}}^{\mathrm{OMA}}$ in 64 are independent of the number of antennas employed at the serving BS, which is due to the non-coherent combining used at the serving $\mathrm{BS} \mathbf{w}_{k}^{\mathrm{H}} \mathbf{g}_{k^{\prime}, l}$, thereby leading to the effective ICI channel becoming equivalent to a single-antenna Rayleigh fading channel. Therefore, all the ergodic sum-rates of NOMA in single-antenna, multi-antenna, and massive-MIMO singlecell systems are degraded upon replacing the noise power $N_{0}$ by $\left(I_{\text {inter }}^{\text {NOMA }}+N_{0}\right)$. On the other hand, since OMA schemes only face a noise power level of $\frac{1}{G} N_{0}$ on each subband, all the ergodic sum-rates of the OMA schemes in singleantenna, multi-antenna, and massive-MIMO single-cell systems are reduced upon substituting the noise power $\frac{1}{G} N_{0}$ by $I_{\text {inter }}^{\mathrm{OMA}}+\frac{1}{G} N_{0}=\frac{1}{G}\left(I_{\text {inter }}^{\mathrm{NOMA}}+N_{0}\right)$.

Given the ICI terms $I_{\text {inter }}^{\mathrm{NOMA}}$ and $I_{\text {inter }}^{\mathrm{OMA}}$, we have the corresponding asymptotic ESGs in single-antenna, multiantenna, and massive-MIMO multi-cell systems as shown from Equation (66) to 70 on the top of next page ${ }^{19}$ where $\psi_{k}{ }^{\prime}=\frac{P_{\max } M}{\sum_{i=k+1}^{K} P_{\max } I_{i}+K\left(I_{\text {inter }}^{\mathrm{NOMA}}+N_{0}\right)}, \quad \xi^{\prime}=\frac{P_{\max } M}{W\left(I_{\text {inter }}^{\mathrm{NOMA}}+N_{0}\right)}$, and $\varpi^{\prime}=\frac{P_{\max }}{\left(1+D_{0}^{\alpha}\right)\left(I_{\text {inter }}^{\text {NOMA }}+N_{0}\right)}$.

In (66)-70, we have mathematically quantified the impact of ICI on the ESG of NOMA over OMA in multi-cell systems. Besides, from (66)-(70), we can observe directly an ESG degradation in multi-cell systems due to ICI, compared to the corresponding ESGs in single-cell systems. In fact, NOMA schemes enable all the users in adjacent cells to simultaneously transmit their signals on the same frequency band and thus the ICI power level in NOMA schemes is substantially higher than that in OMA schemes, as derived in 64). Secondly, it can be observed in Equations (66)-(70) that the ESGs in multi-cell systems can be easily obtained by replacing the noise power $N_{0}$ to the ICI plus noise power $\left(I_{\text {inter }}^{\mathrm{NOMA}}+N_{0}\right)$

\footnotetext{
${ }^{19}$ Note that the performance analysis in multi-cell systems considered in this work does not assume a large SNR. Therefore, the ESG analyses in Equations 66)- 70 are accurate for the whole SNR regime, as shown in Fig. 8 in Section VII of this paper.
}

in corresponding ESGs of single-cell systems. In fact, OMA schemes endure not only $\frac{1}{G}$ of noise power, but also $\frac{1}{G}$ of ICI power, as derived in 64, compared to NOMA schemes. Therefore, the performance analyses in single-cell systems can be directly applied to multi-cell systems via increasing $N_{0}$ to $\left(I_{\text {inter }}^{\mathrm{NOMA}}+N_{0}\right)$. We note that without the proposed asymptotic approximations, obtaining such important and interesting insights would not have been possible. More insights can be observed from the numerical results in Section VII-D. Upon utilizing the coordinate signal processing among multiple cells [55], the ICI power can be effectively suppressed, which may prevent the ESG degradation, when extending NOMA from single-cell to multi-cell systems.

Remark 9: In practice, users' channel ordering might keep changing for different channel realizations. In this work, we assume that the users are indexed based on their instantaneous channel gains, i.e., $\left\|\mathbf{h}_{1}\right\| \geq\left\|\mathbf{h}_{2}\right\|, \ldots, \geq\left\|\mathbf{h}_{K}\right\|$, and the SIC detection order at the $\mathrm{BS}$ is $1,2, \ldots, K$ correspondingly. It implies that the variant of users' ordering changes the SIC detection order at the BS. However, the analytical results obtained in this paper are applicable to the case of varying user ordering. Let us explain the logic in the following.

Firstly, the ergodic sum-rates in Equations (13) and 24) do not change when changing the users' ordering for SISONOMA and MIMO-NOMA systems, respectively. It is because the instantaneous sum-rates of both SISO-NOMA and MIMONOMA in (6) and (20), respectively, are independent of the SIC detection order and thus independent of the users' ordering [27]. Therefore, we can safely assume i.i.d. channel distribution for all the NOMA users and neglect the changing of users' ordering for each instantaneous channel realization. As a result, the obtained analytical results for single-antenna and multi-antenna systems are still applicable to the case when the ordering of $K$ users' channels keeps changing.

Secondly, for massive MIMO systems, the instantaneous sum-rate of $m$ MIMO-NOMA in 43 indeed depends on the instant channel ordering. However, as we apply the theory of order statistics for performance analysis in $m$ MIMO-NOMA systems, the ergodic sum-rates in (44) and (48) are obtained via averaging w.r.t. the $k$-th largest user's channel distribution, $\forall k=1,2, \ldots, K$, but are not determined by which user is indexed as the $k$-th user. Therefore, the obtained results for massive MIMO systems are also applicable to the case when users' channel ordering keeps changing.

Thirdly, we note that if the obtained results in single-cell systems are applicable to the case of varying users' order, the obtained results in multi-cell systems are also applicable to the case when the user ordering keeps changing. In fact, the main difference between single-cell and multi-cell systems is ICI. However, as all the $K$ users in adjacent cell inflicts the ICI to each user homogeneously in the desired cell and no joint-cell processing is performed, the ICI power does not depends on the user ordering.

In summary, the obtained results in all the considered scenarios in this work are applicable to the case when the channel ordering keeps changing. 


$$
\begin{aligned}
& \lim _{K \rightarrow \infty}{\overline{G^{\mathrm{SISO}}}}^{\prime} \approx \ln \left(1+\frac{P_{\max }}{\left(D+D_{0}\right)\left(I_{\text {inter }}^{\mathrm{NOMA}}+N_{0}\right)} \sum_{n=1}^{N} \frac{\beta_{n}}{c_{n}}\right) \\
& -\frac{1}{\left(D+D_{0}\right)} \sum_{n=1}^{N} \beta_{n} e^{\frac{c_{n}\left(I_{\text {inter }}^{\mathrm{NOMA}}+N_{0}\right)}{P_{\max }}} \mathcal{E}_{1}\left(\frac{c_{n}\left(I_{\text {inter }}^{\mathrm{NOMA}}+N_{0}\right)}{P_{\max }}\right) \\
& \lim _{K \rightarrow \infty} \overline{G_{\mathrm{FDMA}-\mathrm{ZF}}^{\mathrm{MIMO}}} \approx M \ln \left(1+\frac{P_{\max }}{\left(D+D_{0}\right)\left(I_{\mathrm{inter}}^{\mathrm{NOMA}}+N_{0}\right)} \sum_{n=1}^{N} \frac{\beta_{n}}{c_{n}}\right) \\
& -\frac{M}{\left(D+D_{0}\right)} \sum_{n=1}^{N} \beta_{n} e^{\frac{c_{n} M\left(I_{\text {inter }}^{\mathrm{NOMA}}+N_{0}\right)}{P_{\max }}} \mathcal{E}_{1}\left(\frac{c_{n} M\left(I_{\mathrm{inter}}^{\mathrm{NOMA}}+N_{0}\right)}{P_{\max }}\right) \\
& \lim _{K \rightarrow \infty}{\overline{G_{\mathrm{FDMA}-\mathrm{MRC}}^{\mathrm{MIMO}}}}^{\prime} \approx M \ln \left(1+\frac{P_{\max }}{\left(D+D_{0}\right)\left(I_{\mathrm{inter}}^{\mathrm{NOMA}}+N_{0}\right)} \sum_{n=1}^{N} \frac{\beta_{n}}{c_{n}}\right) \\
& -\frac{1}{\left(D+D_{0}\right)} \sum_{n=1}^{N} \beta_{n}\left(\frac{\left(\frac{\left(I_{\text {inter }}^{\mathrm{NOMA}}+N_{0}\right) c_{n}}{P_{\max }}\right)^{M}}{\Gamma(M)} \mathcal{G}_{2,3}^{3,1}\left(\begin{array}{c}
-M,-M+1 \\
-M,-M, 0
\end{array} \mid \frac{\left(I_{\text {inter }}^{\mathrm{NOMA}}+N_{0}\right) c_{n}}{P_{\max }}\right)\right) \\
& \lim _{K \rightarrow \infty, M \rightarrow \infty}{\overline{G_{D>D_{0}}^{m \text { MIMO}}}}^{\prime} \approx \lim _{K \rightarrow \infty, M \rightarrow \infty} \sum_{k=1}^{K}\left(\begin{array}{c}
K \\
k
\end{array}\right) \frac{k}{D+D_{0}} \sum_{n=1}^{N} \beta_{n} \\
& \times\left[\ln \left(1+\frac{\psi_{k}{ }^{\prime}}{c_{n}}\right)-\delta \varsigma \ln \left(1+\frac{\xi^{\prime}}{c_{n}}\right)\right]\left(\frac{\phi_{n}^{2}-D_{0}^{2}}{D^{2}-D_{0}^{2}}\right)^{k-1}\left(\frac{D^{2}-\phi_{n}^{2}}{D^{2}-D_{0}^{2}}\right)^{K-k} \\
& \lim _{K \rightarrow \infty, M \rightarrow \infty}{\overline{G_{D=D_{0}}^{m \mathrm{MIMO}}}}^{\prime} \approx \frac{M}{\varpi^{\prime} \delta}\left[\ln \left(1+\varpi^{\prime} \delta+\varpi^{\prime}\right)\left(1+\varpi^{\prime} \delta+\varpi^{\prime}\right)\right. \\
& \left.-\ln \left(1+\varpi^{\prime} \delta\right)\left(1+\varpi^{\prime} \delta\right)-\ln \left(1+\varpi^{\prime}\right)\left(1+\varpi^{\prime}\right)\right]-\varsigma M \ln \left(1+\frac{\varpi^{\prime}}{\varsigma}\right)
\end{aligned}
$$

\section{Some Consistency Discussions}

In this section, we want to provide some discussions on the consistency among the results obtained in previous sections. Also, the consistency between our main results with the existing related works are discussed.

Although distinctive signal detection methods are adopted in different scenarios in Sections III, IV, and V, they are consistent in the sense that they all focus on analyzing and characterizing the ESG of NOMA over OMA. The obtained results are related and their comparison can unveil interesting insights. Firstly, as shown in (38), the asymptotic ESG of MIMO-NOMA over MIMO-OMA using FDMA-ZF is $M$ times higher than that in single-antenna systems, when the BS is equipped with $M$ antennas serving a sufficiently large number of users $K$. Additionally, the asymptotic ESG of $m$ MIMO-NOMA over $m$ MIMO-OMA also increases linearly with $M$. This is mainly due to the additional spatial DoF offered by the additional antennas at the BS. Secondly, in all the considered scenarios, the ESG of NOMA over OMA increases with increasing the system SNR, while it vanishes in the low-SNR regime. In fact, as NOMA exploits the power domain for multi-user multiplexing, a high power is beneficial to improve the performance of NOMA. Thirdly, the ESG of NOMA over OMA in all the considered scenario increases with the number of users $K$, owing to the small-scale fading gain and large-scale near-far gain. Fourthly, it can be observed from all the considered scenarios with both single-cell and multi-cell deployments that a large cell size facilitates a higher ESG of NOMA over OMA. This is due to the benefit of the increased large-scale near-far gain for a larger cell size.

Most of the existing works focused on the outage performance analysis of NOMA systems [41], [44], [45], [48], [53] or on the analytical proof of superiority of NOMA over OMA [40], [46], [47]. However, the ESG of NOMA over OMA has not been mathematically quantified. Our work provides a generalized framework which offers analytical expressions for not only explaining the results of existing works, e.g. [62], [63], but also unveiling new insights on the performance gain of NOMA over OMA in different scenarios. In [62], Ding et al. analytically shown that the performance gain of NOMA over OMA can be enlarged when pairing two users with distinctive channel conditions as a NOMA group. This is consistent with our conclusion in this paper where a larger normalized cell size enables a higher ESG of NOMA over OMA. Furthermore, in [63], Ding et al. analytically demonstrated that the performance gain of NOMA over OMA increases with the number of users $K$ when pairing user 1 and user $K$ as a NOMA group. It is also consistent with our obtained conclusion that the ESG increases with $K$. However, we note that both [62], [63] only considered a two-user NOMA case, while our work consider multiple users in a NOMA group, which is more general. 


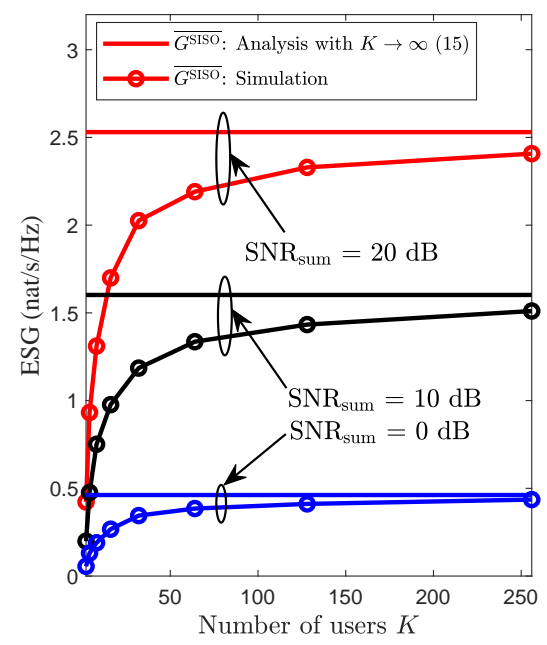

(a) Single-antenna systems

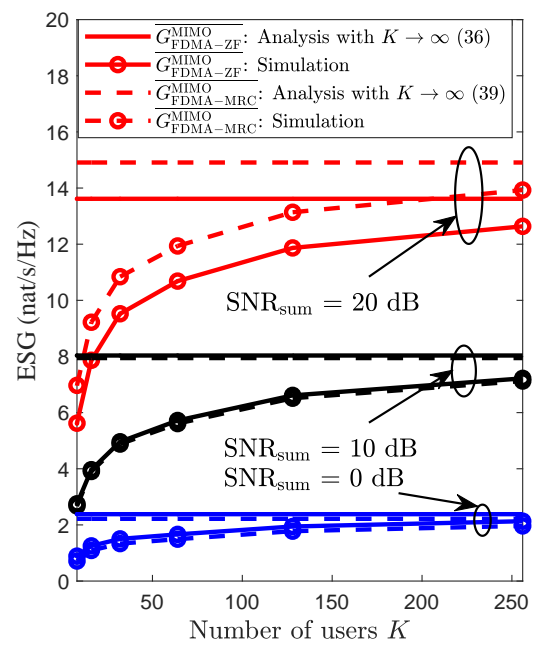

(b) Multi-antenna systems

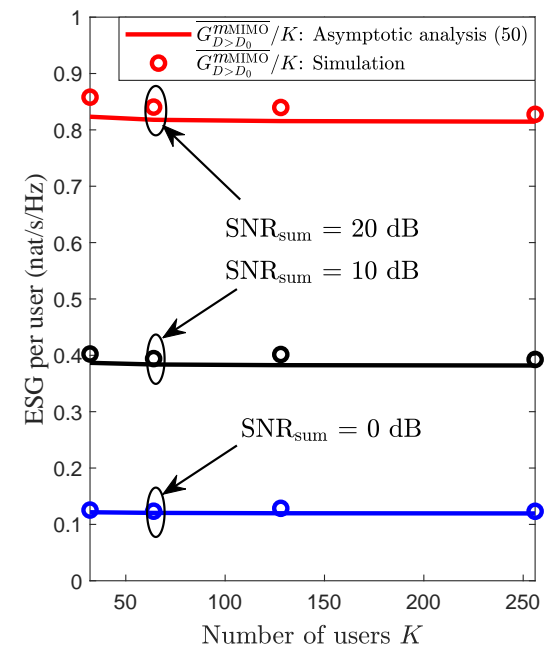

(c) Massive-antenna systems

Fig. 5. The ESG of NOMA over OMA versus the number of users $K$. The normalized cell size is $\eta=10$ and the average received sum SNR is $\mathrm{SNR}_{\mathrm{sum}}=[0,10,20] \mathrm{dB}$. For the considered MIMO-NOMA and MIMO-OMA systems in Fig. 5(b) we have $M=4$. For the considered $m$ MIMO-NOMA and $m$ MIMO-OMA systems in Fig. 5(c) the number of antennas equipped at the BS is adjusted according to the number of users $K$ based on $M=K \delta$ with $\delta=\frac{1}{2}$.

TABLE III

System Parameters USEd In Simulations

\begin{tabular}{l|r}
\hline Inner cell radius, $D_{0}$ & $50 \mathrm{~m}$ \\
\hline Outer cell radius, $D$ & {$[50,200,500] \mathrm{m}$} \\
\hline Normalized cell size, $\eta$ & {$[1,4,10]$} \\
\hline Number of users, $K$ & $2 \sim 256$ \\
\hline Number of receive antennas at BS, $M$ & $1 \sim 128$ \\
\hline Path loss exponent, $\alpha$ & 3.76 \\
\hline Noise power, $N_{0}$ & $-80 \mathrm{dBm}$ \\
\hline System SNR, SNR & $0 \sim 40 \mathrm{~dB}$ \\
\hline Ratio $\varsigma=\frac{W}{M}$ for $m$ MIMO-OMA & $\frac{1}{16}$ \\
\hline Ratio $\delta=\frac{M}{K}$ for $m$ MIMO-NOMA & $\frac{1}{2}$ \\
\hline
\end{tabular}

\section{Simulations}

In this section, we use simulations to evaluate our analytical results. In the single-cell systems considered, the inner cell radius is $D_{0}=50 \mathrm{~m}$ and the outer cell radius is given by $D=$ $[50,200,500] \mathrm{m}$, which corresponds to the cases of normalized cell sizes given by $\eta=[1,4,10]$, respectively. The number of users $K$ ranges from 2 to 256 and the number of antennas employed at the BS $M$ ranges from 1 to 128 . The path loss exponent is $\alpha=3.76$ according to the 3GPP path loss model [58]. The noise power is set as $N_{0}=-80 \mathrm{dBm}$. To emphasize the effect of cell size on the ESG of NOMA over OMA, in the simulations of the single-cell systems, we characterize the system's SNR with the aid of the total average received SNR of all the users at the BS as follows [35]:

$$
\mathrm{SNR}_{\text {sum }}=\frac{P_{\max }}{N_{0}} \overline{|h|^{2}}=\frac{P_{\max }}{N_{0}} \frac{\overline{\|\mathbf{h}\|^{2}}}{M},
$$

where $\overline{|h|^{2}}$ and $\overline{\|\mathbf{h}\|^{2}}$ are given by (12) and (25), respectively. The total transmit power $P_{\max }$ is adjusted adaptively for different cell sizes to satisfy $\mathrm{SNR}_{\text {sum }}$ in (71) ranging from $0 \mathrm{~dB}$ to $40 \mathrm{~dB}$. In the mMIMO-OMA system considered, we set the ratio between the group size and the number of antennas to $\varsigma=\frac{W}{M}=\frac{1}{16}$, hence we can assume that the favorable propagation conditions prevail in the spirit of [68]. Additionally, in the $m$ MIMO-NOMA system considered, the ratio between the number of receiver antennas at the BS and the number of serving users is fixed as $\delta=\frac{M}{K}=\frac{1}{2}$. The important system parameters adopted in our simulations are summarized in Table III] The specific simulation setups for each simulation scenario are shown under each figure. All the simulation results in this paper are obtained by averaging the system performance over both small-scale fading and largescale fading.

\section{A. ESG versus the Number of Users in Single-cell Systems}

Fig. 5 illustrates the ESG of NOMA over OMA versus the number of users in the single-antenna, multi-antenna, and massive-MIMO single-cell systems. In both Fig. 5(a) and Fig. 5 (b), we can observe that the ESG increases with the number of users $K$ and eventually approaches the asymptotic results derived for $K \rightarrow \infty$. This is because upon increasing the number of users, the heterogeneity in channel gains among users is enhanced, which leads to an increased near-far gain. As shown in Fig. 5(c), for massive-MIMO systems, the asymptotic ESG per user derived in 50 closely matches with the simulations even for moderate numbers of users and SNRs. Although (54) is derived for massive-MIMO systems with $D=D_{0}$, in Fig. 5(c), we can observe a constant ESG per user in massiveMIMO systems with $D>D_{0}$. In other words, the insights obtained from the massive-MIMO systems with $D=D_{0}$ are also applicable to the scenarios of $D>D_{0}$. Compared to the ESG in the single-antenna systems of Fig. 5(a) the ESG in the multi-antenna systems of Fig. 5(b) is substantially increased due to the extra spatial DoF offered by additional antennas at the BS. Moreover, it can be observed in Fig. 5(b) that we 


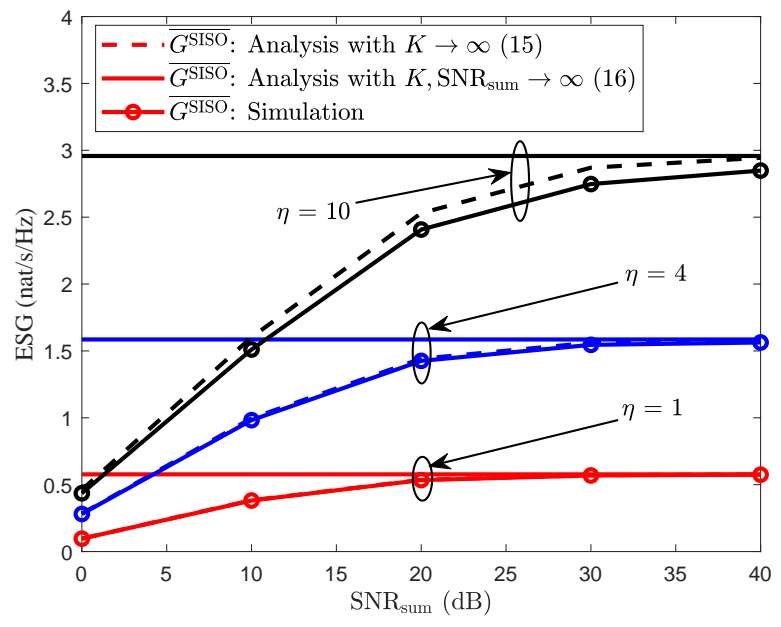

(a) ESG of SISO-NOMA over SISO-OMA.

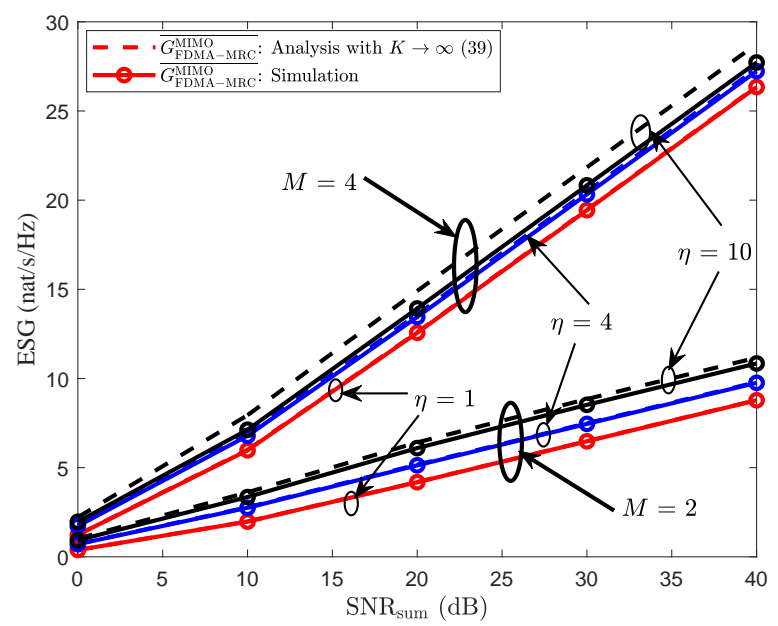

(c) ESG of MIMO-NOMA over MIMO-OMA with FDMA-MRC.

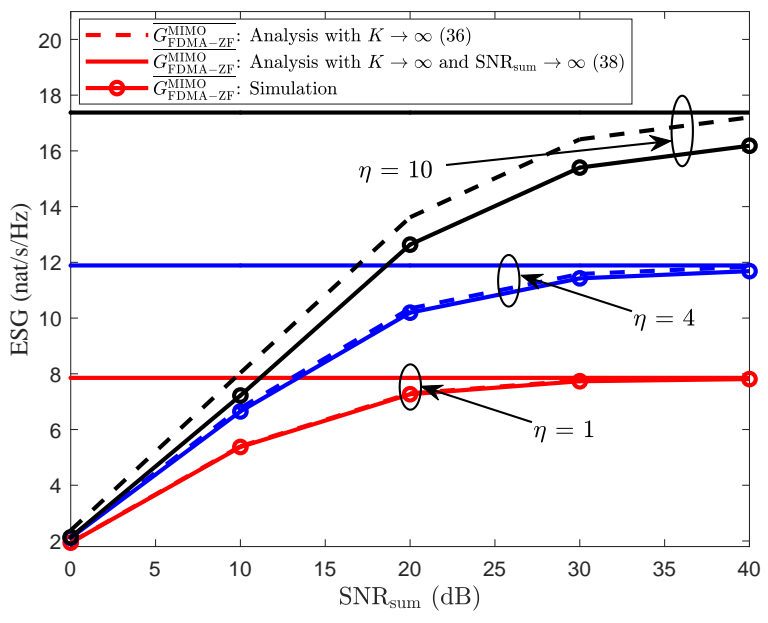

(b) ESG of MIMO-NOMA over MIMO-OMA with FDMA-ZF.

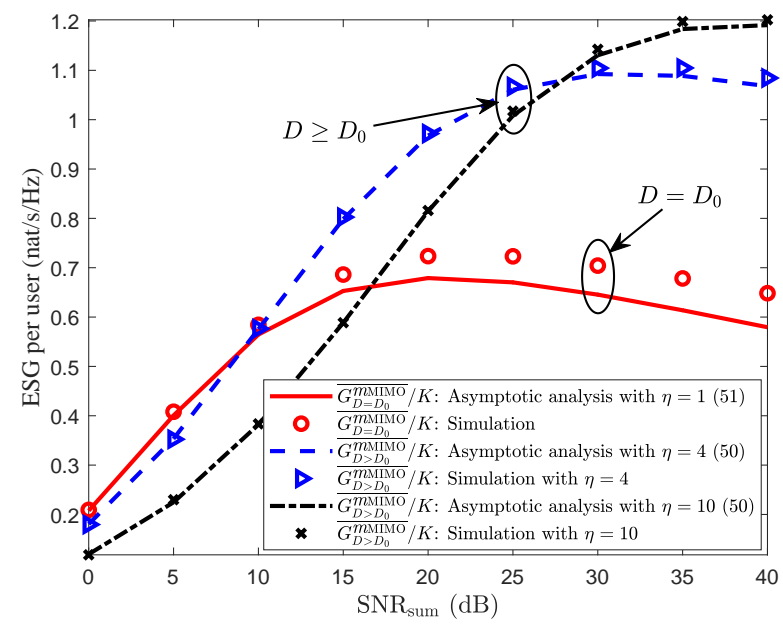

(d) ESG of $m$ MIMO-NOMA over $m$ MIMO-OMA.

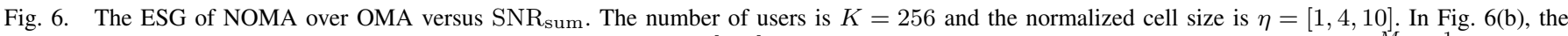
number of antennas equipped at the BS $M=4$, while we have $M=[2,4]$ in Fig. 6(c) In Fig. 6(d) we have $M=128$ such that $\delta=\frac{M}{K}=\frac{1}{2}$.

have $\lim _{K \rightarrow \infty} \overline{G_{\mathrm{FDMA}-\mathrm{ZF}}^{\mathrm{MIMO}}}>\lim _{K \rightarrow \infty} \overline{G_{\mathrm{FDMA}-\mathrm{MRC}}^{\mathrm{MIMO}}}$ in the lowSNR case, while $\lim _{K \rightarrow \infty} \overline{G_{\mathrm{FDMA}-\mathrm{MF}}^{\mathrm{MIMO}}}<\lim _{K \rightarrow \infty} \overline{G_{\mathrm{FDMA}-\mathrm{MRC}}^{\mathrm{MIMO}}}$ in the high-SNR case. This is because ZF detection outperforms MRC detection in the high-SNR regime for the MIMO-OMA system considered, while it becomes inferior to MRC detection in the low-SNR regime. Furthermore, we can observe a higher ESG in Fig. 5(a), Fig. 5(b) and Fig. 5(c) for the high-SNR case, e.g. $\mathrm{SNR}_{\text {sum }}=20 \mathrm{~dB}$. This is due to the powerdomain multiplexing of NOMA, which enables multiple users to share the same time-frequency resource and motivates a more efficient exploitation of the power resource.

\section{B. ESG versus the SNR in Single-cell Systems}

Fig. 6 depicts the ESG of NOMA over OMA versus the system's SNR SNR $_{\text {sum }}$ within the range of $\mathrm{SNR}_{\text {sum }}=[0,40]$ $\mathrm{dB}$ in the single-antenna, multi-antenna, and massive-MIMO single-cell systems. We can observe that the simulation results match closely our asymptotic analyses in all the considered cases. Besides, by increasing the system SNR, the ESGs seen in Fig. 6(a) and Fig. 6(b) increase monotonically and approach the asymptotic analyses results derived in the highSNR regime. In other words, the ESGs seen in Fig. 6(a) and Fig. 6(b) are bounded from above even if $P_{\max } \rightarrow \infty$. This is because there is no DoF gain in the ESG of NOMA over OMA in the pair of scenarios considered. By contrast, as derived in 41), the $(M-1)$-fold DoF gain in the ESG of MIMO-NOMA over MIMO-OMA with FDMA-MRC enables the ESG to increase linearly with the system's SNR in dB in the high-SNR regime, as shown in Fig. 6(c). Furthermore, a higher number of antennas provides a larger DoF gain, which leads to a steeper slope of ESG versus the system SNR in dB. In contrast to Fig. 6(a), Fig. 6(b), and Fig. 6(c) the ESG of $m \mathrm{MIMO}-\mathrm{NOMA}$ over $m \mathrm{MIMO}-\mathrm{OMA}$ recorded in Fig. 6(d) first increases and then decreases with the system SNR, especially for a small normalized cell size. In fact, 


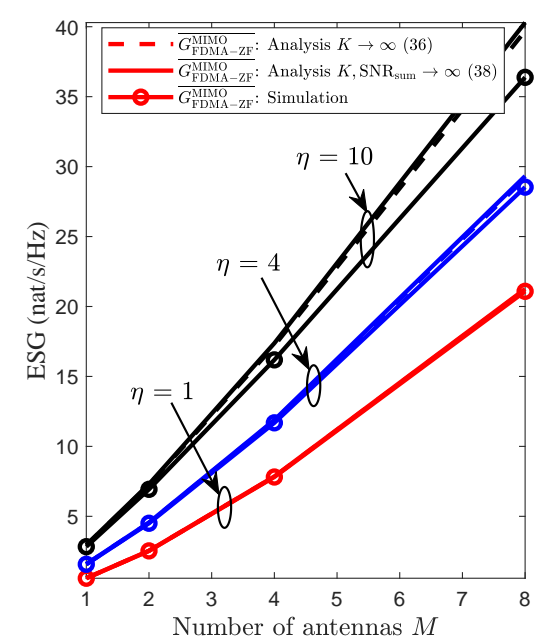

(a) ESG of MIMO-NOMA over MIMO-OMA with FDMA-ZF.
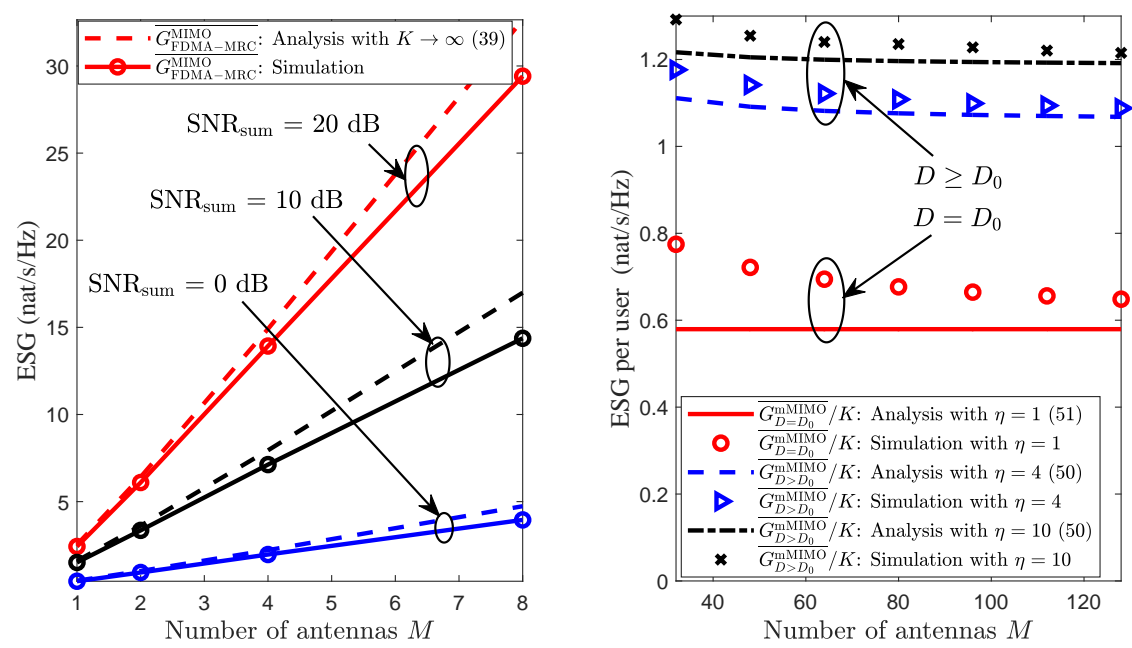

(b) ESG of MIMO-NOMA over MIMO-OMA with (c) ESG of $m$ MIMO-NOMA over $m$ MIMO-OMA FDMA-MRC.

Fig. 7. The ESG of NOMA over OMA versus the number of antennas $M$. The number of users is $K=256$ in Fig. 7(a) and Fig. 7(b) The normalized cell size is $\eta=[1,4,10]$ in Fig. 7(a) and Fig. 7(c) while it is set as $\eta=[10]$ in Fig. 7(b) The average received sum SNR is SNR sum $=[0,10,20]$ dB in Fig. 7(b) while it is set as SNR sum $^{2}=[40]$ dB in Fig. 7(a) and Fig. 7(c) In multi-antenna systems in Fig. 7(a) and Fig. 7(b) the number of antennas $M$ equipped at the BS ranges from 1 to 8 . In massive-MIMO systems in Fig. 7(c) $M$ ranges from 32 to 128 , and the number of users $K$ is adjusted according to $M$ based on $K=\frac{M}{\delta}$ with $\delta=\frac{1}{2}$.

the $m$ MIMO-NOMA system relying on MRC-SIC detection becomes interference-limited in the high-SNR regime, while the $m$ MIMO-OMA system remains interference-free, since favorable propagation conditions prevail for $\varsigma=\frac{W}{M} \ll 1$. As a result, upon increasing the system SNR, the increased IUI of the mMIMO-NOMA system considered neutralizes some of its ESG over the mMIMO-OMA system, particularly for a small cell size associated with a limited large-scale near-far gain.

On the other hand, it is worth noticing in Fig. 6(a) that if all the users are randomly distributed on a circle when $D=D_{0}=50 \mathrm{~m}$, i.e., $\eta=1$, then we have an ESG of about $0.575 \mathrm{nat} / \mathrm{s} / \mathrm{Hz}$ at $\mathrm{SNR}_{\text {sum }}=40 \mathrm{~dB}$ for SISO-NOMA compared to SISO-OMA. This again verifies the accuracy of the small-scale fading gain $\gamma$ derived in (16). Furthermore, we can observe in Fig. 6(a), Fig. 6(b), and Fig. 6(c), that a larger normalized cell size $\eta$ results in a higher performance gain, which is an explicit benefit of the increased large-scale near-far gain $\vartheta(\eta)$. By contrast, in Fig. 6(d), a larger cell size facilitates a higher ESG but only in the high-SNR regime, while a smaller cell size can provide a larger ESG in the low to moderate-SNR regime. In fact, due to the large number of antennas, the IUI experienced in the $m$ MIMO-NOMA system is significantly reduced compared to that in single-antenna and multi-antenna systems. As a result, in the low to moderateSNR regime, the $m$ MIMO-NOMA system considered may be noise-limited rather than interference-limited, which is in line with the single-antenna and multi-antenna systems. For instance, the noise degrades the achievable rates of the celledge users more severely compared to the impact of IUI in the $m$ MIMO-NOMA system, especially for large normalized cell sizes. Therefore, the large-scale near-far gain cannot be fully exploited in the low to moderate-SNR regime in the massiveMIMO systems. Moreover, it can be observed in Fig. 6(d) that the ESG increases faster for a larger normalized cell size $\eta$. This is due to the enhanced large-scale near-far gain observed for a larger cell size, which enables NOMA to exploit the power resource more efficiently.

\section{ESG versus the Number of Antennas $M$ in Single-cell Systems}

Fig. 7 illustrates the ESG of NOMA over OMA versus the number of antennas $M$ employed at the BS in multiantenna and massive-MIMO systems. It can be observed that the simulation results closely match our asymptotic analyses for all the simulation scenarios. In particular, observe for the ESG of MIMO-NOMA over MIMO-OMA with FDMA-ZF in Fig. 7(a) that as predicted in (38), the asymptotic ESG $G^{\text {SISO }}$ of single-antenna systems is increased by $M$, when an $M$-antenna array is employed at the BS. More importantly, a larger normalized cell size $\eta$ enables a steeper slope in the ESG versus the number of antennas $M$, which is due to the increased large-scale near-far gain $\vartheta(\eta)$, as shown in (37). Apart from the linearly increased component of ESG vesus $M$, an additional power gain factor of $\ln (M)$ can also be observed in Fig. 7(a) as derived in (38). Observe the ESG of MIMONOMA over MIMO-OMA with FDMA-MRC in Fig. 7(b) that the ESG grows linearly versus $M$ due to the $(M-1)$-fold of DoF gain and the corresponding slope becomes higher for a higher system SNR, as seen in (41). The ESG per user seen in Fig. 7(c) for massive-MIMO systems remains almost constant upon increasing $M$, which matches for our asymptotic analysis in (54), and is also consistent with the results of Fig. 5(c) for the fixed ratio $\delta=\frac{M}{K}$. Furthermore, we can observe that a 


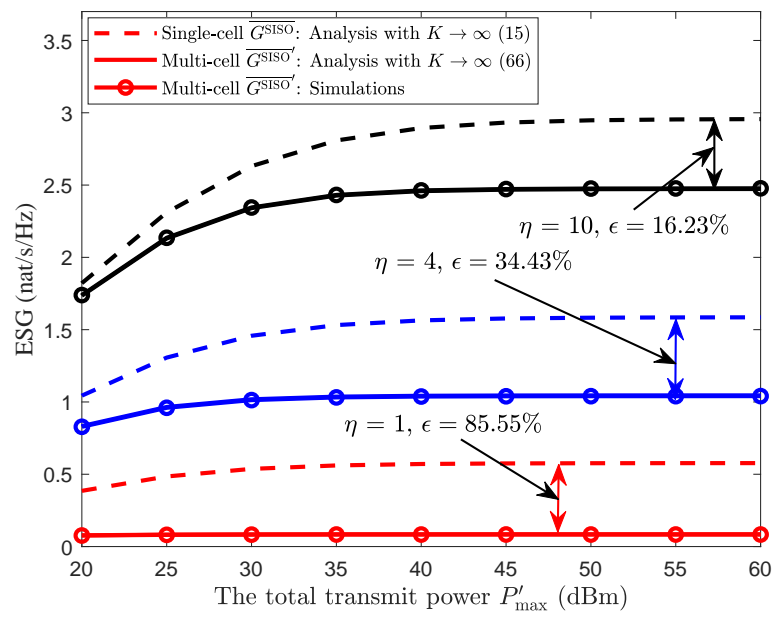

(a) The ESG of SISO-NOMA over SISO-OMA.

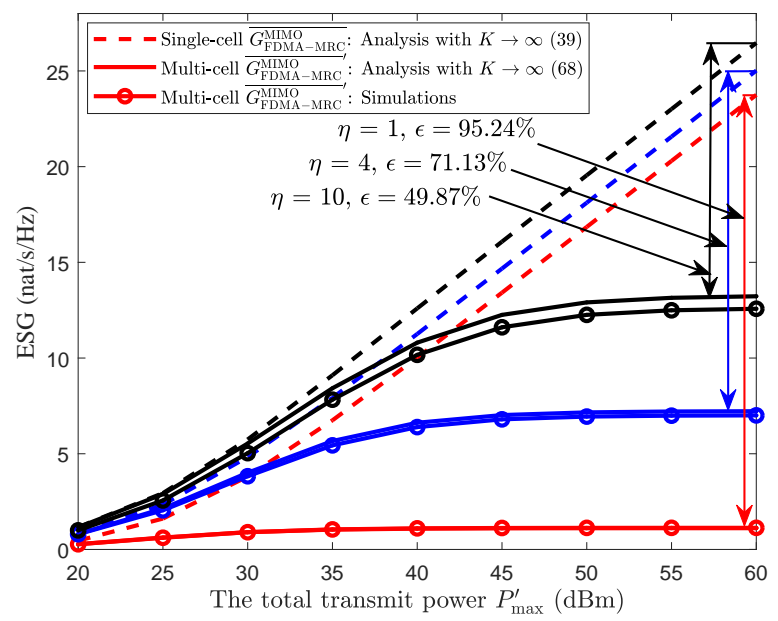

(c) ESG of MIMO-NOMA over MIMO-OMA with FDMA-MRC.

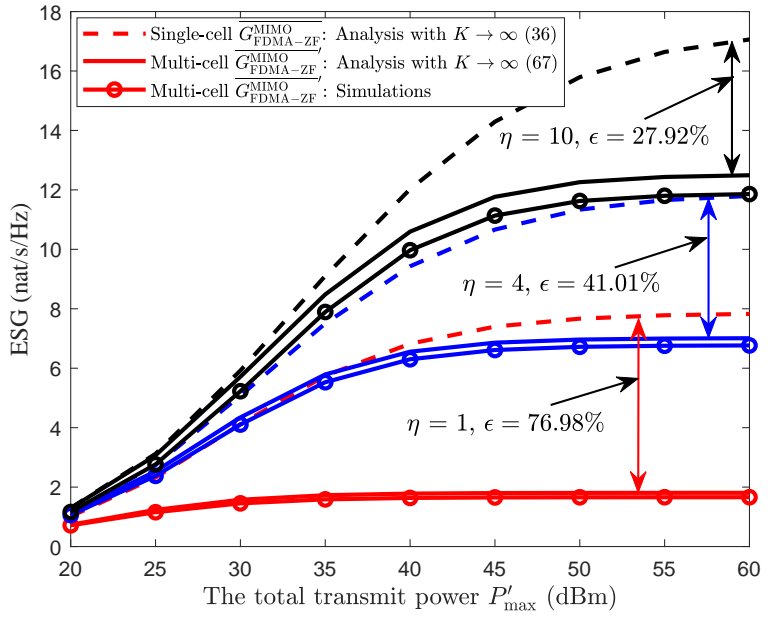

(b) ESG of MIMO-NOMA over MIMO-OMA with FDMA-ZF.

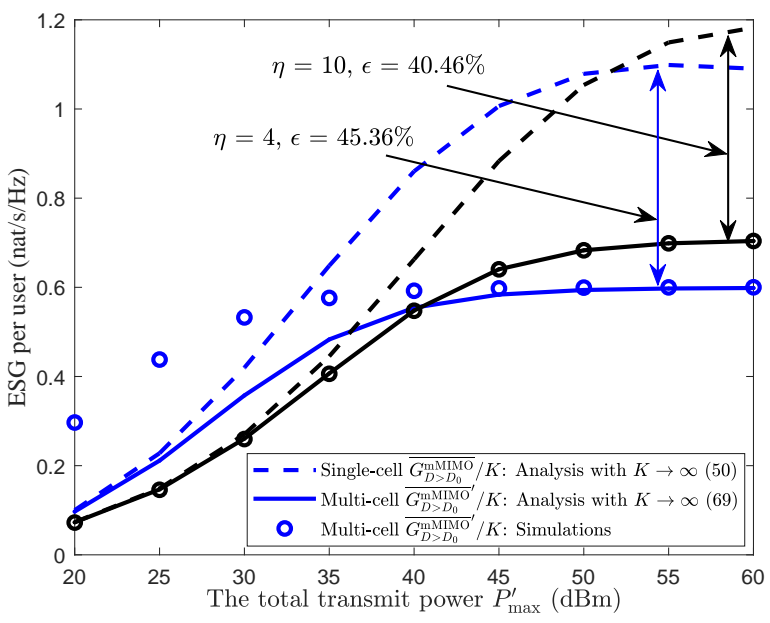

(d) ESG of mMIMO-NOMA over mMIMO-OMA.

Fig. 8. ESG versus the total transmit power $P_{\max }^{\prime}$ in multi-cell systems. The normalized cell size is $\eta=[1,4,10]$ in Fig. 8(a) Fig. 8(b) and 8(c) while it is set as $\eta=[4,10]$ in Fig. 8(d) The number of antennas equipped at each BS is $M=1$ in Fig. 8(a) and $M=4$ in Fig. 8(b) as well as Fig. 8(c) In Fig. $8(\mathrm{~d})$ the number of antennas equipped at each BS is adjusted based on the number of users in each cell via $M=\lceil K \delta\rceil$ and the group size of the considered $m$ MIMO-OMA system is $W=\lceil\varsigma M\rceil$. The ESG degradations due to the ICI are denoted by double-sided arrows.

large cell size offers a higher ESG per user due to the improved large-scale near-far gain.

\section{ESG versus the Total Transmit Power in Multi-cell Systems}

In a multi-cell system, we consider a high user density scenario within a large circular area with the radius of $D_{1}=5$ $\mathrm{km}$ and the user density of $\rho=1000$ devices per $\mathrm{km}^{2}$. As a result, the total number of users in the multi-cell system considered is $K^{\prime}=\left\lceil\rho \pi D_{1}^{2}\right\rceil$. Then, the number of users in each cell is given by $K=\left\lceil\rho \pi D^{2}\right\rceil$ with $D$ in the unit of $\mathrm{km}$. Meanwhile, the number of adjacent cells $L$ can be obtained by $L=\left\lceil\frac{K^{\prime}-K}{K}\right\rceil$, so that all the $K^{\prime}$ users can be covered. Furthermore, the $K^{\prime}$ users in all the cells share a given total transmit power and the total transmit power $P_{\max }^{\prime}$ of $(L+1)$ cells is within the range spanning from $20 \mathrm{dBm}$ to
$60 \mathrm{dBm}^{20}$ In this section, we also consider an equal power allocation among multiple cells and an equal power allocation among users within each cell, i.e., we have $P_{\max }=\frac{P_{\max }^{\prime}}{L+1}$ and $p_{k^{\prime}, l}=\frac{P_{\max }}{K}$. All the other simulation parameters are the same as those adopted in the single-cell systems.

In contrast to the single-cell systems, the ESG versus the total transmit power $P_{\max }^{\prime}$ trends are more interesting, which is due to the less straightforward impact of ICI on the performance gain of NOMA over OMA in multi-cell systems. Fig. 8 shows the ESG of NOMA over OMA versus the total transmit power $P_{\max }^{\prime}$ in single-antenna, multi-antenna, and

${ }^{20}$ Since there are a larger number of users deployed in the considered area, we set a large power budget for all the users in the considered multi-cell system. 
TABLE IV

COMPARISON ON ESG (NAT/S/Hz) OF NOMA OVER OMA IN THE CONSIDERED SCENARIOS. THE SYSTEM SETUP IS $K=256, D=200 \mathrm{M}, \eta=4$, AND $M=4$ FOR MULTI-ANTENNA SYSTEMS.

\begin{tabular}{c|cc|cc}
\hline & \multicolumn{2}{|c|}{$\mathrm{SNR}_{\text {sum }}=0 \mathrm{~dB}$} & \multicolumn{2}{c}{$\mathrm{SNR}_{\text {sum }}=10 \mathrm{~dB}$} \\
\hline & Single-cell & Multi-cell & Single-cell & Multi-cell \\
\hline Single-antenna & 0.281 & 0.2639 & 0.983 & 0.7973 \\
Multi-antenna & 2.114 & 2.0179 & 6.65 & 5.4113 \\
Massive-MIMO (ESG per user) & 0.1796 & 0.1702 & 0.5765 & 0.4490 \\
\hline
\end{tabular}

massive-MIMO ${ }^{21}$ multi-cell systems. The analytical results in single-cell systems are also shown for comparison. As shown in Fig. 8, we can observe that the performance gains of NOMA over OMA are degraded upon extending NOMA from singlecell systems to multi-cell systems. This is because the ICI of NOMA systems is contributed by all the users in adjacent cells, while only a subset of users introduce ICI to the desired cell in OMA systems. Additionally, it can be observed from Fig. 8 that the ESG degradation becomes more severe with increasing the total transmit power. Furthermore, in the highSNR regime, the ESG degradation is larger for the case with a smaller cell size. Note that the performance degradation $\epsilon$ in Fig. 8(c) is calculated at $p_{\max }=60 \mathrm{dBm}$, as the ESGs in single-cell systems are not saturated in the high-SNR regime. This is because the ICI power increases with either increasing the total transmit power budget or decreasing the cell size. For the ease of illustration, we define the normalized performance degradation of the ESG in multi-cell systems compared to that in single-cell systems as $\epsilon=\frac{\bar{G}-\bar{G}^{\prime}}{\bar{G}}$, where $\bar{G}$ denotes the ESG in single-cell systems and $\bar{G}^{\prime}$ denotes the ESG in multicell systems. It can be observed that the ESG degradation is more severe for a small normalized cell size $\eta$, because multi-cell systems suffer from a more severe ICI for smaller cell sizes due to a shorter inter-site distance. Therefore, the system performance becomes saturated even for a moderate system power budget in the case of a smaller cell size.

It is worth noting that the ESG of MIMO-NOMA over MIMO-OMA with FDMA-MRC is saturated in multi-cell systems in the high transmit power regime, as shown in Fig. 8(c), which is different from the trends seen for single-cell systems in Fig. 6(c). In fact, the $(M-1)$-fold DoF gain in the ESG of MIMO-NOMA over MIMO-OMA with FDMA-MRC in single-cell systems derived in (41) can only be achieved in the high-SNR regime. However, due to the lack of joint multi-cell signal processing to mitigate the ICI, the multicell system becomes interference-limited upon increasing the total transmit power. Therefore, the multi-cell system actually operates in the low-SINR regime, which does not facilitate the exploitation of the DoF gain in single-cell systems.

Remark 10: The comparison of the ESG (nat $/ \mathrm{s} / \mathrm{Hz}$ ) results of NOMA over OMA in all the scenarios considered is summarized in Table IV] We consider a practical operation setup with $K=256, D=200 \mathrm{~m}, \eta=4$, and $M=4$ for the

\footnotetext{
${ }^{21}$ Note that, for the considered massive-MIMO multi-cell system, a small cell size leads to a small number of users $K$ in each cell and thus results in a small number of antennas $M$ due to the fixed ratio $\delta=\frac{M}{K}$. This is contradictory to our assumption of $K \rightarrow \infty$ and $M \rightarrow \infty$. Therefore, we only consider the normalized cell size of $\eta=[4,10]$ for massive-MIMO multi-cell systems in Fig. 8(d)
}

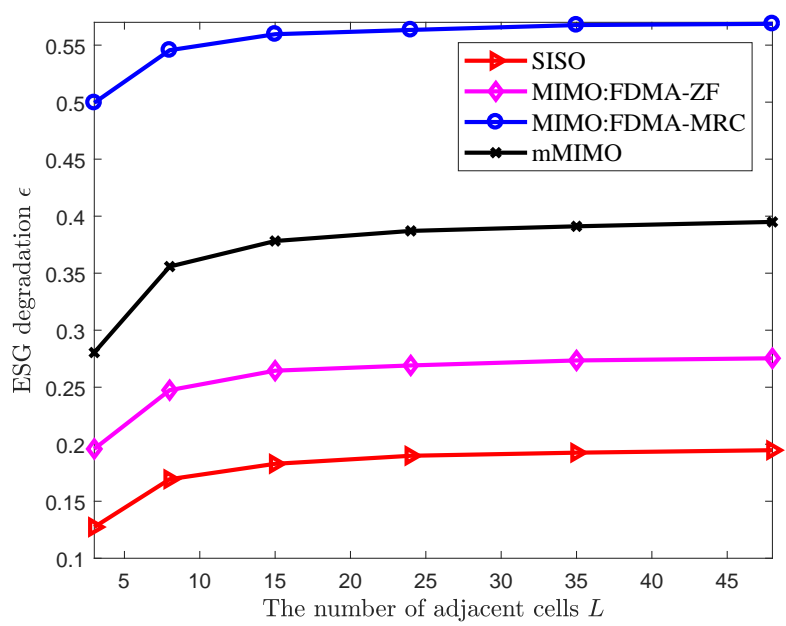

Fig. 9. ESG degradation $\epsilon$ versus the number of adjacent cells $L$. The cell size is fixed as $D=500 \mathrm{~m}$, the number of users in each cell is $K=\left\lceil\rho \pi D^{2}\right\rceil$, and the total transmit power for each cell is $P_{\max }=46 \mathrm{dBm}$. The number of antennas equipped at each BS is $M=4$ for MIMO systems. In the considered $m$ MIMO system, the number of antennas equipped at each BS is adjusted based on the number of users in each cell via $M=\lceil K \delta\rceil$ and the group size of the considered $m$ MIMO-OMA system is $W=\lceil\varsigma M\rceil$.

multi-antenna systems. For fair comparison, the total transmit power $P_{\max }^{\prime}$ in multi-cell systems is adjusted for ensuring that the total average received SNR $\mathrm{SNR}_{\text {sum }}$ at the serving BS is identical to that in single-cell systems. Note that the row of massive-MIMO in Table IV quantifies the ESG per user of $m$ MIMO-NOMA over $m$ MIMO-OMA, which is consistent with Fig. 6(d), Fig. 7(c) and Fig. 8(d) We can observe that the ESG remains a near-constant at the low-SNR of $\mathrm{SNR}_{\text {sum }}=0$ $\mathrm{dB}$ when extending NOMA from single-cell systems to multicell systems, while the ESG degrades substantially at the highSNR of $\mathrm{SNR}_{\text {sum }}=10 \mathrm{~dB}$. In fact, the limited transmit power budget in the low-SNR regime in adjacent cells only leads to a low ICI level at the serving BS, which avoids a significant ESG degradation, when applying NOMA in multi-cell systems.

\section{E. The Impact of the Number of Adjacent Cells}

As demonstrated in Fig. 8, the ESG of NOMA over OMA is degraded when extending NOMA from single-cell to multicell systems due to the more severe ICI faced by NOMA. To further illustrate the impact of the number of adjacent cells $L$ on the ESG degradation, we consider a multi-cell system having a variable number of adjacent cells $L$. In our simulations, we fix the cell size to $D=500 \mathrm{~m}$ and the number of adjacent cells is determined by the area considered, i.e., we have $L=\left\lceil\frac{K^{\prime}-K}{K}\right\rceil=\left\lceil\frac{D_{1}^{2}-D^{2}}{D^{2}}\right\rceil$ given a constant user density. 


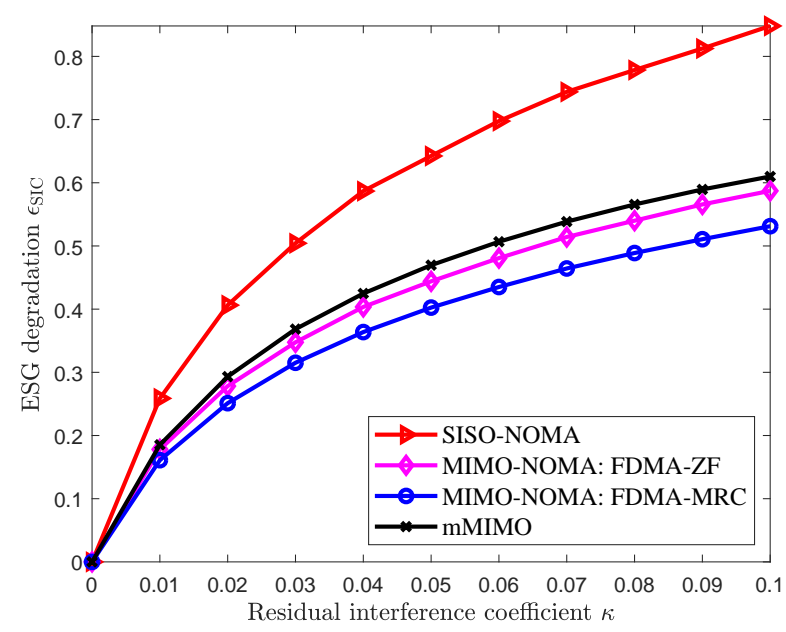

Fig. 10. ESG degradation $\epsilon_{\mathrm{SIC}}$ versus the residual interference coefficien $\kappa$. The cell size is fixed as $D=500 \mathrm{~m}$, the number of users in each cell is $K=256$, and the system SNR is $\mathrm{SNR}_{\text {sum }}=20 \mathrm{~dB}$. The number of antennas equipped at each $\mathrm{BS}$ is $M=4$ for MIMO systems. In the considered $m$ MIMO system, the number of antennas equipped at each BS is fixed as $M=\lceil K \delta\rceil=128$ and the group size of the considered $m$ MIMO-OMA system is $W=\lceil\varsigma M\rceil$.

Fig. 9 illustrates the ESG degradation $\epsilon$ versus the number of adjacent cells $L$ for single-antenna, multi-antenna, and massive-MIMO base stations. We can observe that the ESG degradation increases upon increasing the number of adjacent cells $L$ due to have more severe ICI faced by the desired cell. Additionally, the ESG degradation becomes saturated upon increasing $L$. This is because the additionally introduced cells corresponding to increasing $L$ are far away from the desired cell for a large $L$. Therefore, the additional ICI imposed on the BS in the desired cell is reduced upon increasing $L$ and thus the ESG degradation almost does not change with further introducing more adjacent cell. It implies that in practice only several tiers of adjacent cells have significant impacts on the performance gain of NOMA of OMA in multi-cell systems.

\section{F. The Impact of the SIC Detection Error}

In this treatise, we assume having perfect SIC for simplifying our performance analysis. In practice, SIC detection suffers from error propagation, when the detection error of the preceding interference cancelation affects the current interference cancelation [88]. Indeed, realistic imperfect SIC has a significant impact on the performance gain of NOMA over OMA, especially for a large NOMA group size $K$. At the time of writing, it remains an open problem to characterize the impact of imperfect SIC. This is because imperfect SIC is influenced by many factors, such as the coding/modulation strategy, the channel estimation accuracy, and the hardwarerelated restrictions. Hence, it is a challenge to model their impact on SIC detection [88]. Therefore, we rely on simulations to investigate the impact of realistic imperfect SIC on the performance gain of NOMA over OMA via employing a simplified residual interference model introduced in [88], [89]. In particular, the impact of the preceding imperfect interference cancelation on the current interference cancelation is modelled by a residual interference source, which is assumed to be a linear function of the received signal power [88], [89]. We note that the adopted residual interference model is a simplified model for illustrating the impact of SIC detection error via simulations in this work. A non-linear model can characterize the impact of imperfect SIC more accurately, since the residual interference also depends on the received power difference among NOMA users in practice.

According to Equation (4), the instantaneous achievable data rate of user $k$ in the SISO-NOMA system considered associated with imperfect SIC is given by

$$
R_{k}^{\mathrm{SISO}-\mathrm{NOMA}}=\ln \left(1+\frac{p_{k}\left|h_{k}\right|^{2}}{\sum_{i=k+1}^{K} p_{i}\left|h_{i}\right|^{2}+\sum_{i=1}^{k-1} \kappa_{i} p_{i}\left|h_{i}\right|^{2}+N_{0}}\right),
$$

where $0 \leq \kappa_{i}<1$ denotes the coefficient between the residual interference power and the received signal power for user $i$, namely the residual interference coefficient, which can be obtained by long-term measurement [88]. Similarly, in conjunction with imperfect SIC detection, the instantaneous achievable data rates of user $k$ in the MIMO-NOMA and $m$ MIMO-NOMA systems considered are given by Equations (73) and 74 on the top of next page, respectively. Note that Equation (74) is a straightforward extension of Equation (42). The derivation details of Equation (73) are given in the Appendix F.

To demonstrate the impact of SIC detection errors on the performance gain of NOMA over OMA, we further define the ESG degradation $\epsilon_{\mathrm{SIC}}=\frac{\bar{G}_{\text {Perfect }}-\bar{G}_{\text {Imperfect }}}{\bar{G}_{\text {Perfect }}}$, where $\bar{G}_{\text {Perfect }}$ and $\bar{G}_{\text {Imperfect }}$ denotes the ESG with perfect SIC detection and imperfect SIC detection, respectively. When $\epsilon_{\mathrm{SIC}}=0$, we have $\bar{G}_{\text {Perfect }}=\bar{G}_{\text {Imperfect }}$, i.e., there is no ESG degradation even with imperfect SIC detection. Additionally, we note that $\epsilon_{\mathrm{SIC}} \geq 1$ implies $\bar{G}_{\text {Imperfect }} \leq 0$, which means that NOMA is inferior to OMA in this case.

Based on the achievable data rate expressions (72), (73), and (74), we can perform simulations for evaluating the impact of imperfect SIC on the performance gain of NOMA over OMA. We note that the SIC detection imperfection only introduces additional intra-cell interference but does not affect the intercell interference. Thus, one can imagine that the insights obtained for multi-cell systems still hold even for imperfect SIC detection. Therefore, we only perform simulations for single-cell systems with imperfect SIC detection.

Fig. 10 depicts the ESG degradation $\epsilon_{\mathrm{SIC}}$ versus the residual interference coefficient $\kappa$. In our simulations, we assume that $\kappa_{1}=\kappa_{2}, \ldots,=\kappa_{K}=\kappa$. Furthermore, we consider a fixed cell size of $D=500 \mathrm{~m}$, a fixed number of users $K=256$, and a fixed system SNR of $\mathrm{SNR}_{\text {sum }}=20 \mathrm{~dB}$ for our simulations. We can observe that the higher the residual interference coefficient, the more severe the ESG degradation becomes. In fact, the residual interferences from previous stages of interference cancelation accumulate and jointly affect the current interference cancelation. Therefore, even a small value of $\kappa=0.05$ leads to a severe ESG degradation, i.e., the SIC error propagation degrades the performance gain of NO- 


$$
\begin{aligned}
R_{k}^{\mathrm{MIMO}-\mathrm{NOMA}} & =\ln \left|\mathbf{I}_{M}+\frac{1}{N_{0}} \sum_{i=k}^{K} p_{i} \mathbf{h}_{i} \mathbf{h}_{i}^{\mathrm{H}}+\frac{1}{N_{0}} \sum_{i=1}^{k-1} \kappa_{i} p_{i} \mathbf{h}_{i} \mathbf{h}_{i}^{\mathrm{H}}\right|-\ln \left|\mathbf{I}_{M}+\frac{1}{N_{0}} \sum_{i=k+1}^{K} p_{i} \mathbf{h}_{i} \mathbf{h}_{i}^{\mathrm{H}}+\frac{1}{N_{0}} \sum_{i=1}^{k-1} \kappa_{i} p_{i} \mathbf{h}_{i} \mathbf{h}_{i}^{\mathrm{H}}\right| \\
R_{k}^{m \text { MIMO-NOMA }} & =\ln \left(1+\frac{p_{k}\left\|\mathbf{h}_{k}\right\|^{2}}{\sum_{i=k+1}^{K} p_{i}\left\|\mathbf{h}_{i}\right\|^{2}\left|\mathbf{e}_{k}^{\mathrm{H}} \mathbf{e}_{i}\right|^{2}+\sum_{i=1}^{k-1} \kappa_{i} p_{i}\left\|\mathbf{h}_{i}\right\|^{2}\left|\mathbf{e}_{k}^{\mathrm{H}} \mathbf{e}_{i}\right|^{2}+N_{0}}\right)
\end{aligned}
$$

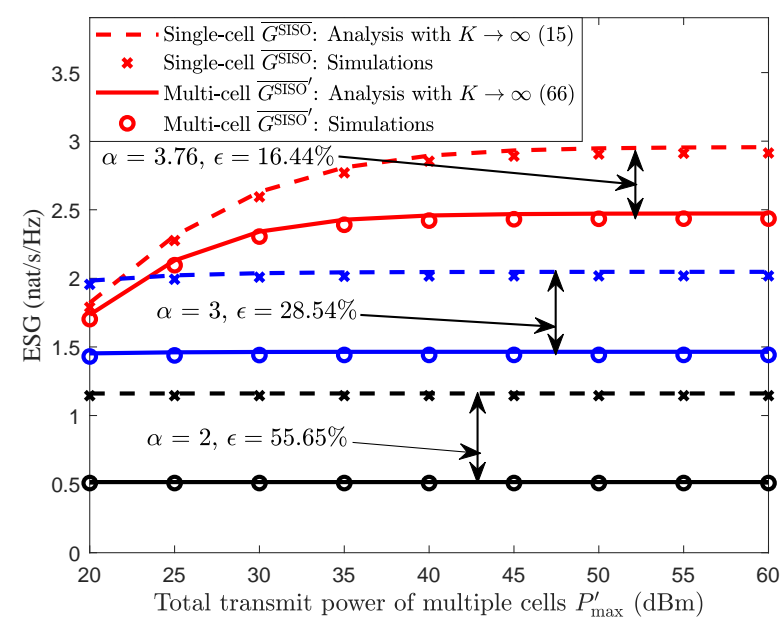

Fig. 11. ESG versus the total transmit power $P_{\max }^{\prime}$ in multi-cell systems with different path loss exponents $\alpha=[3.76,3,2]$. The normalized cell size is $\eta=10$. The ESG degradations due to the ICI are denoted by double-sided arrows.

MA over OMA. Additionally, the ESG degradation of SISO systems is substantially higher than that of the other systems. In fact, in single-antenna systems, the residual interference signal is fully imposed on the current stage of interference cancelation, while it is projected onto the currently detected user's channel owing to the multiple antennas employed at the BS. As a result, SISO-NOMA systems are more sensitive to SIC detection error propagation than MIMO-NOMA and $m$ MIMO-NOMA systems.

\section{G. The Impact of the Path Loss Exponent}

We note that the obtained analytical results in this paper are applicable to any other values of $\alpha$, as it has been treated as a fixed constant in our performance analysis. For illustration, we simulate the ESG of SISO-NOMA over SISO-OMA in both single-cell and multi-cell systems with different path loss exponents $\alpha$, as shown in Fig. 11. Note that the path loss exponent, $\alpha$, only determines the signal power attenuation along the propagation distance and has a homogeneous effect on all the antennas at the BS. As a result, the simulation results in a single-antenna system is sufficient to illustrate the impact of $\alpha$ on ESG of NOMA over OMA. In Fig. 11, we can observe that the ESG of NOMA over OMA for a larger $\alpha$ outperforms that for a smaller $\alpha$ in both single-cell and multicell systems. In fact, as demonstrated in 16 , the performance gain of NOMA over OMA partially arises from the large-scale near-far gain. Besides, as the path loss exponent $\alpha$ determines the slope of path loss w.r.t. the distance between the transmitter and receiver, one can imagine that a smaller $\alpha$ results in a smaller large-scale near-far gain. As a result, the ESG of NOMA over OMA degrades with decreasing $\alpha$. Moreover, we can observe that the smaller the path loss exponent $\alpha$, the larger the ESG degradation when extending NOMA from single-cell systems to multi-cell systems. This is due to the fact that the ICI in multi-cell systems becomes more severe with reducing $\alpha$, which results in a more severe ESG degradation.

\section{CONCLUSIONS AND FUTURE WORK}

In this paper, we investigated the ESG in uplink communications attained by NOMA over OMA in single-antenna, multiantenna, and massive-MIMO systems with both single-cell and multi-cell deployments. In the single-antenna single-cell system considered, the ESG of NOMA over OMA was quantified and two types of gains were identified in the ESG derived, i.e., the large-scale near-far gain and the small-scale fading gain. The large-scale near-far gain increases with the cell size, while the small-scale fading gain is a constant of $\gamma=0.57721$ $\mathrm{nat} / \mathrm{s} / \mathrm{Hz}$ in Rayleigh fading channels. Additionally, we unveiled that the ESG of SISO-NOMA over SISO-OMA can be increased by $M$ times upon using $M$ antennas at the BS, owing to the extra spatial DoF offered by additional antennas. In the massive-MIMO single-cell system considered, the ESG of NOMA over OMA increases linearly both with the number of users and with the number of antennas at the BS. The analytical results derived for single-cell systems were further extended to multi-cell systems via characterizing the effective ICI channel distribution and by deriving the ICI power. We found that a larger cell size is preferred by NOMA for both single-cell and multi-cell systems, due to the enhanced largescale near-far gain and reduced ICI, respectively. Extensive simulation results have shown the accuracy of our performance analyses and confirmed the insights provided above.

In this paper, as a first attempt to unveil fundamental insights on the performance gain of NOMA over OMA, we considered the ideal case associated with perfect CSI and error-propagation-free SIC detection at the BS. In practice, it is difficult to acquire the perfect CSI due to channel estimation errors, feedback delays, and/or quantization errors. Similarly, the error propagation during SIC detection is usually also inevitable in practice. In our future work, we will investigate the ESG of NOMA over OMA both in the face of imperfect CSI and error propagation during SIC detection. 


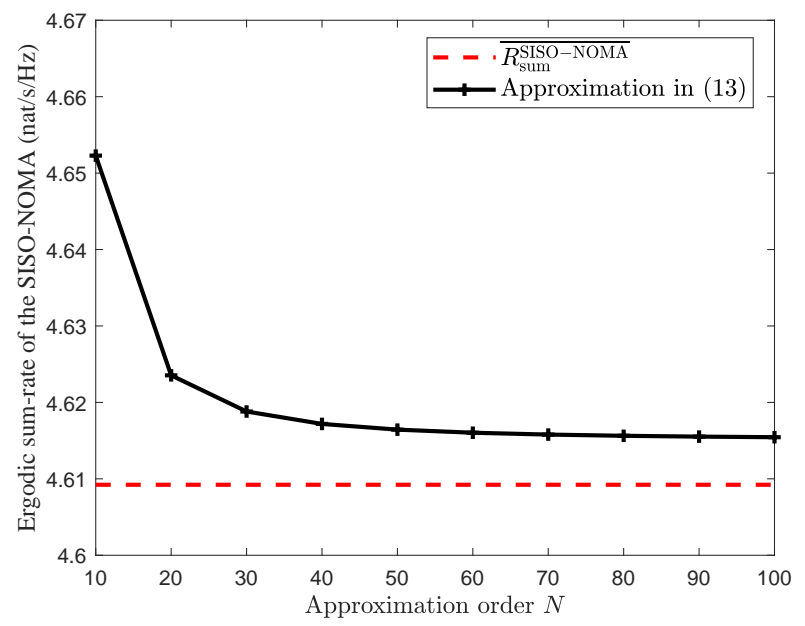

Fig. 12. The approximation error of the ergodic sum-rate of SISO-NOMA.

\section{ACKNOWLEDGEMENT}

The authors would like to thank Prof. Ping Li from the City University of Hong Kong for valuable discussions during this work.

\section{APPENDIX}

\section{A. Proof of Lemma 1}

Since all the users are scattered uniformly across the pair of concentric rings between the inner radius of $D_{0}$ and the outer radius of $D$ in Fig. 1, the CDF of the channel gain $|h|^{2}$ is given by

$$
F_{|h|^{2}}(x)=\int_{D_{0}}^{D}\left(1-e^{-\left(1+z^{\alpha}\right) x}\right) f_{d}(z) d z
$$

where $f_{d}(z)=\frac{2 z}{D^{2}-D_{0}^{2}}, D_{0} \leq z \leq D$, denotes the probability density function (PDF) for the random distance $d$. The Gaussian-Chebyshev quadrature approximation is a polynomial approximation with a high accuracy [76]. For a given function $g(x)$, its definite integral can be represented by [76]

$$
\begin{aligned}
\int_{a}^{b} g(x) d x & =\frac{b-a}{2} \sum_{n=1}^{N} \frac{\pi}{N} \sqrt{1-x_{n}^{2}} g\left(\frac{b-a}{2} x+\frac{b+a}{2}\right) \\
& +\mathcal{O}_{N}(\xi)
\end{aligned}
$$

and the approximation error is given by

$$
\mathcal{O}_{N}(\xi)=\frac{2 \pi}{2^{2 N}(2 N) !}\left(\sqrt{1-\xi^{2}} g\left(\frac{b-a}{2} \xi+\frac{b+a}{2}\right)\right)^{(2 N)},
$$

for some $-1<\xi<1$, where $(\cdot)^{(2 N)}$ denotes the $2 N$ times derivative operation. We can observe from (77) that for a smooth function $g(x)$, the error term $\mathcal{O}_{N}(\xi)$ diminishes with increasing $N$. As a result, the approximation accuracy in ergodic sum-rate of SISO-NOMA in (13) also becomes higher with increasing $N$, as shown in Fig. 12 .

\section{B. Proof of Theorem 1}

To facilitate the proof, we first consider a virtual system whose capacity serves as an upper bound to that of the system in (1). In particular, the virtual system is the uplink of a $K$ user $M \times M$ MIMO system with $M$ antennas employed at each user and the BS. We assume that, in the virtual $K$-user $M \times M$ MIMO system, each user faces $M$ parallel subchannels with identical subchannel gain $\left\|\mathbf{h}_{k}\right\|$, i.e., the channel matrix between user $k$ and the BS is $\left\|\mathbf{h}_{k}\right\| \mathbf{I}_{M}$. As a result, the signal received at the $B S$ is given by

$$
\tilde{\mathbf{y}}=\sum_{k=1}^{K} \sqrt{p_{k}}\left\|\mathbf{h}_{k}\right\| \mathbf{I}_{M} \tilde{\mathbf{x}}_{k}+\mathbf{v}
$$

where $\tilde{\mathbf{x}}_{k}=\mathbf{u}_{k} x_{k} \in \mathbb{C}^{M \times 1}$ denotes the transmitted signal after preprocessing by a precoder $\mathbf{u}_{k} \in \mathbb{C}^{M \times 1}$. We note that the precoder should satisfy the constraint $\operatorname{Tr}\left(\mathbf{u}_{\mathrm{k}} \mathbf{u}_{\mathrm{k}}^{\mathrm{H}}\right) \leq 1$, so that $\mathrm{E}\left\{\tilde{\mathbf{x}}_{k}^{\mathrm{H}} \tilde{\mathbf{x}}_{k}\right\} \leq \mathrm{E}\left\{x_{k}^{2}\right\}=1$. Additionally, in the virtual $K$-user $M \times M$ MIMO system, the subchannel gain between user $k$ and the BS is forced to be identical as $\left\|\mathbf{h}_{k}\right\|$, where $\left\|\mathbf{h}_{k}\right\|$ is the corresponding channel gain value between user $k$ and the BS in the original $K$-user $1 \times M$ MIMO system in (1). Furthermore, we consider an arbitrary but the identical power allocation strategy $\mathbf{p}=\left[p_{1}, \ldots, p_{K}\right]$ as that of our original system in (1) during the following proof. Upon comparing (1) and (78), we can observe that the specific choice of the precoder $\mathbf{u}_{k}=\frac{\mathbf{h}_{k}}{\left\|\mathbf{h}_{k}\right\|}$ in (78) would result in an equivalent system to that in (1). In other words, the capacity of the system in (78) serves as an upper bound to that of the system in (1), i.e., we have:

$$
\begin{aligned}
& R_{\text {sum }}^{\mathrm{MIMO}-\mathrm{NOMA}} \stackrel{(a)}{=} C(M, K, \mathbf{p}, \mathbf{H}) \leq C\left(M^{2}, K, \mathbf{p}, \widetilde{\mathbf{H}}\right) \\
& =\max _{\operatorname{Tr}\left(\mathbf{u}_{k} \mathbf{u}_{k}^{\mathrm{H}}\right) \leq 1} \ln \left|\mathbf{I}_{M}+\frac{1}{N_{0}} \sum_{k=1}^{K} p_{k}\left\|\mathbf{h}_{k}\right\|^{2} \mathbf{I}_{M} \mathbf{u}_{k} \mathbf{u}_{k}^{\mathrm{H}} \mathbf{I}_{M}^{\mathrm{H}}\right| \\
& =M \ln \left(1+\frac{1}{M N_{0}} \sum_{k=1}^{K} p_{k}\left\|\mathbf{h}_{k}\right\|^{2}\right),
\end{aligned}
$$

where $C(M, K, \mathbf{p}, \mathbf{H})$ denotes the capacity for the uplink $K$-user $1 \times M$ MIMO system in (1) for a channel matrix $\mathbf{H}=\left[\mathbf{h}_{1}, \ldots, \mathbf{h}_{K}\right]$ and power allocation $\mathbf{p}$. Furthermore, $C\left(M^{2}, K, \mathbf{p}, \widetilde{\mathbf{H}}\right)$ denotes the capacity of the virtual $K$-user $M \times M$ MIMO system in (78) associated with a channel matrix $\widetilde{\mathbf{H}}=\left[\left\|\mathbf{h}_{1}\right\| \mathbf{I}_{M}, \ldots,\left\|\mathbf{h}_{K}\right\| \mathbf{I}_{M}\right]$, while $\mathbf{p}$ is the value as in (1). The achievable sum-rate $R_{\text {sum }}^{\mathrm{MIMO}-\mathrm{NOMA}}$ is given in 20 and the equality $(a)$ in 79 is obtained by a capacity-achieving MMSE-SIC [27].

Now, to prove the asymptotic tightness of the upper bound considered in (79), we have to consider a lower bound of the achievable sum-rate in 20, and prove that asymptotically the upper bound and the lower bound converge to the same expression. For the uplink $K$-user $1 \times M$ MIMO system in (1), we assume that all the users transmit their signals subject to the power allocation $\mathbf{p}=\left[p_{1}, \ldots, p_{K}\right]$ and the BS utilizes an MRC-SIC receiver to retrieve the messages of all the $K$ users. Then the achievable rate for user $k$ of the MIMO-NOMA 
system using the MRC-SIC receiver is given by:

$$
R_{k, \text { MRC-SIC }}^{\mathrm{MIMO}}=\ln \left(1+\frac{p_{k}\left\|\mathbf{h}_{k}\right\|^{2}}{\sum_{i=k+1}^{K} p_{i}\left\|\mathbf{h}_{i}\right\|^{2}\left|\mathbf{e}_{k}^{\mathrm{H}} \mathbf{e}_{i}\right|^{2}+N_{0}}\right),
$$

where $\mathbf{e}_{k}=\frac{\mathbf{h}_{k}}{\left\|\mathbf{h}_{k}\right\|}$ denotes the channel direction of user $k$. Then, it becomes clear that the achievable sum-rate of the MIMO-NOMA system using the MRC-SIC receiver serves as a lower bound to the channel capacity in (20), i.e., we have

$$
R_{\mathrm{sum}, \mathrm{MRC}-\mathrm{SIC}}^{\mathrm{MIMO}}=\sum_{k=1}^{K} R_{k, \mathrm{MRC}-\mathrm{SIC}}^{\mathrm{MIMO}-\mathrm{NOMA}} \leq R_{\mathrm{sum}}^{\mathrm{MIMO}-\mathrm{NOMA}} .
$$

Through the following theorem and corollaries, we first characterize the statistics of $\mathbf{e}_{k}$ as well as $\left|\mathbf{e}_{k}^{\mathrm{H}} \mathbf{e}_{i}\right|^{2}$ and derive the asymptotic achievable sum-rate of MIMO-NOMA employing an MRC-SIC receiver. Then, we show that the upper bound considered in 79) and the lower bound of (81) will asymptotically converge to the same limit for $K \rightarrow \infty$.

Lemma 3: For $\mathbf{h}_{k} \sim \mathcal{C N}\left(\mathbf{0}, \frac{1}{1+d_{k}^{\alpha}} \mathbf{I}_{M}\right)$, the normalized random vector (channel direction) $\mathbf{e}_{k}=\frac{\mathbf{h}_{k}}{\left\|\mathbf{h}_{k}\right\|}$ is uniformly distributed on a unit sphere in $\mathbb{C}^{M}$.

Proof: According to the system model of $\mathbf{h}_{k}=\frac{\mathbf{g}_{k}}{\sqrt{1+d_{k}^{\alpha}}}$ with $\mathbf{g}_{k} \sim \mathcal{C N}\left(\mathbf{0}, \mathbf{I}_{M}\right)$, we have $\mathbf{h}_{k} \sim \mathcal{C N}\left(\mathbf{0}, \frac{1}{1+d_{k}^{\alpha}} \mathbf{I}_{M}\right)$. The distribution of $\mathbf{e}_{k}$ can be proven by exploiting the orthogonal-invariance of the multivariate normal distribution. In particular, for any orthogonal matrix $\mathbf{Q}$, we have $\mathbf{Q} \mathbf{h}_{k} \sim$ $\mathcal{C N}\left(\mathbf{0}, \frac{1}{1+d_{k}^{\alpha}} \mathbf{I}_{M}\right)$, which means that the distribution of $\mathbf{h}_{k}$ is invariant to rotations (orthogonal transform). Then, $\mathbf{e}_{k}=$ $\frac{\mathbf{Q h}_{k}}{\left\|\mathbf{Q} \mathbf{h}_{k}\right\|}=\frac{\mathbf{Q h}_{k}}{\left\|\mathbf{h}_{k}\right\|}$ is also invariant to rotation. Meanwhile, we have $\left\|\mathbf{e}_{k}\right\|=1$ for sure. Therefore, $\mathbf{e}_{k}$ must be uniformly distributed on a unit sphere on $\mathbb{C}^{M}$.

Corollary 1: The channel direction of user $k, \mathbf{e}_{k}$, is independent of its channel gain $\left\|\mathbf{h}_{k}\right\|$.

Proof: According to Lemma 3 the channel direction $\mathbf{e}_{k}$ is uniformly distributed on a unit sphere on $\mathbb{C}^{M}$, regardless of the value of $\left\|\mathbf{h}_{k}\right\|$. Therefore, $\mathbf{e}_{k}$ is independent of $\left\|\mathbf{h}_{k}\right\|$.

Corollary 2: The mean and covariance matrix of $\mathbf{e}_{k}$ are given by

$$
\mathrm{E}\left\{\mathbf{e}_{k}\right\}=\mathbf{0} \text { and } \mathrm{E}\left\{\mathbf{e}_{k} \mathbf{e}_{k}^{\mathrm{H}}\right\}=\frac{1}{M} \mathbf{I}_{M},
$$

respectively.

Proof: Due to the symmetry of the uniform spherical distribution, $\mathbf{e}_{k}$ and $-\mathbf{e}_{k}$ have the same distribution and thus we have $\mathrm{E}\left\{\mathbf{e}_{k}\right\}=\mathrm{E}\left\{-\mathbf{e}_{k}\right\}$ and hence $\mathrm{E}\left\{\mathbf{e}_{k}\right\}=\mathbf{0}$. For the reason of symmetry, $\mathbf{e}_{k}=\left[e_{k, 1}, \ldots, e_{k, m}, \ldots, e_{k, M}\right]$ and $\mathbf{e}_{k}^{\prime}=\left[e_{k, 1}, \ldots,-e_{k, m}, \ldots, e_{k, M}\right]$ have the same distribution, where $e_{k, m}$ denotes the $m$-th entry in $\mathbf{e}_{k}$. Therefore, we have $\mathrm{E}\left\{e_{k, m} e_{k, n}^{*}\right\}=\mathrm{E}\left\{-e_{k, m} e_{k, n}^{*}\right\}=-\mathrm{E}\left\{e_{k, m} e_{k, n}^{*}\right\}, \forall m \neq n$,

which implies that the covariance terms are zero, i.e., $\mathrm{E}\left\{e_{k, m} e_{k, n}^{*}\right\}=0, \forall m \neq n$. Note that, the zero covariance terms only reflect the lack of correlation between $e_{k, m}$ and $e_{k, n}$, but not their independence. In fact, the entries of $\mathbf{e}_{k}$ are dependent on each other, i.e., increasing one entry will decrease all the other entries due to $\left\|\mathbf{e}_{k}\right\|=1$. As for the variance, since $\mathbf{e}_{k}$ has been normalized, we have

$$
\sum_{m=1}^{M} \mathrm{E}\left\{e_{k, m}^{2}\right\}=\mathrm{E}\left\{\sum_{m=1}^{M} e_{k, m}^{2}\right\}=1 .
$$

Again, based on the symmetry of the uniform spherical distribution, we have $\mathrm{E}\left\{e_{k, m}^{2}\right\}=\mathrm{E}\left\{e_{k, n}^{2}\right\}, \forall m, n$, and hence we have $\mathrm{E}\left\{e_{k, m}^{2}\right\}=\frac{1}{M}$ and $\mathrm{E}\left\{\mathbf{e}_{k} \mathbf{e}_{k}^{\mathrm{H}}\right\}=\frac{1}{M} \mathbf{I}_{M}$. This completes the proof.

Let us now define a scalar random variable as $\nu_{k, i}=$ $\mathbf{e}_{k}^{\mathrm{H}} \mathbf{e}_{i} \in \mathbb{C}$, which denotes the projection of channel direction of user $k$ on the channel direction of user $i$. Note that the random variable $\nu_{k, i}$ can characterize the IUI during MRC in 807. Additionally, thanks to the independence between $\mathbf{e}_{k}$ and $\left\|\mathbf{h}_{k}\right\|, \nu_{k, i}$ is independent of $\left\|\mathbf{h}_{k}\right\|$ and $\left\|\mathbf{h}_{i}\right\|$. The following Lemma characterizes the mean and variance of $\nu_{k, i}$.

Lemma 4: For $\mathbf{h}_{k} \sim \mathcal{C N}\left(\mathbf{0}, \frac{1}{1+d_{k}^{\alpha}} \mathbf{I}_{M}\right)$ and $\mathbf{e}_{k}=\frac{\mathbf{h}_{k}}{\left\|\mathbf{h}_{k}\right\|}$, the random variable $\nu_{k, i}=\mathbf{e}_{k}^{\mathrm{H}} \mathbf{e}_{i}$ has a zero mean and variance of $\frac{1}{M}$.

Proof: In fact, $\nu_{k, i}$ denotes the projection of $\mathbf{e}_{k}$ on $\mathbf{e}_{i}$, where $\mathbf{e}_{k}$ and $\mathbf{e}_{i}$ are uniformly distributed in a unit sphere on $\mathbb{C}^{M}$. Upon fixing one channel direction $\mathbf{e}_{k}$, the conditional mean and variance of $\nu_{k, i}$ are given by

$$
\begin{aligned}
\mathrm{E}\left\{\nu_{k, i} \mid \mathbf{e}_{k}\right\} & =\mathbf{e}_{k}^{\mathrm{H}} \mathrm{E}\left\{\mathbf{e}_{i}\right\}=0 \text { and } \\
\mathrm{E}\left\{\left|\nu_{k, i}\right|^{2} \mid \mathbf{e}_{k}\right\} & =\mathbf{e}_{k}^{\mathrm{H}} \mathrm{E}\left\{\mathbf{e}_{i} \mathbf{e}_{i}^{\mathrm{H}}\right\} \mathbf{e}_{k}=\frac{1}{M},
\end{aligned}
$$

respectively. Since $\mathbf{e}_{k}$ is uniformly distributed, the integral over $\mathbf{e}_{k}$ will not change the mean and variance. Therefore, the mean and variance of $\nu_{k, i}$ are given by

$$
\mathrm{E}\left\{\nu_{k, i}\right\}=0 \text { and } \mathrm{E}\left\{\left|\nu_{k, i}\right|^{2}\right\}=\frac{1}{M},
$$

respectively, which completes the proof.

Now, based on 80 , we have the asymptotic achievable data rate of user $k$ given by Equation (87) on the top of next page. Note that the equality in $(a)$ in 87 holds asymptotically by applying Corollary 1 and Lemma 4 with $K \rightarrow \infty$. In addition, the equality in $(b)$ in 87 holds with $K \rightarrow \infty$ since $\lim _{x \rightarrow 0} \ln (1+M x)=\lim _{x \rightarrow 0} M \ln (1+x)$. As a result, the asymptotic achievable sum-rate in 81 can be obtained by

$$
\lim _{K \rightarrow \infty} R_{\text {sum,MRC-SIC }}^{\mathrm{MIMO}}=\lim _{K \rightarrow \infty} M \ln \left(1+\frac{1}{M N_{0}} \sum_{k=1}^{K} p_{k}\left\|\mathbf{h}_{k}\right\|^{2}\right) .
$$

Now, upon comparing (79), 81, and 88, it can be observed that the upper bound and the lower bound considered converge when $K \rightarrow \infty$. In other words, for any given power allocation strategy $\mathbf{p}=\left[p_{1}, \ldots, p_{K}\right]$, the upper bound in 79 . is asymptotically tight. It completes the proof. 


$$
\begin{aligned}
\lim _{K \rightarrow \infty} R_{k, \text { MRC-SIC }}^{\text {MIMO-NOMA }} & =\lim _{K \rightarrow \infty} \ln \left(1+\frac{p_{k}\left\|\mathbf{h}_{k}\right\|^{2}}{\sum_{i=k+1}^{K} p_{i}\left\|\mathbf{h}_{i}\right\|^{2}\left|\nu_{k, i}\right|^{2}+N_{0}}\right) \stackrel{(a)}{=} \lim _{K \rightarrow \infty} \ln \left(1+\frac{p_{k}\left\|\mathbf{h}_{k}\right\|^{2}}{\sum_{i=k+1}^{K} p_{i}\left\|\mathbf{h}_{i}\right\|^{2} \frac{1}{M}+N_{0}}\right) \\
& \stackrel{(b)}{=} \lim _{K \rightarrow \infty} M \ln \left(1+\frac{p_{k}\left\|\mathbf{h}_{k}\right\|^{2} \frac{1}{M}}{\sum_{i=k+1}^{K} p_{i}\left\|\mathbf{h}_{i}\right\|^{2} \frac{1}{M}+N_{0}}\right)
\end{aligned}
$$

\section{Proof of Lemma 2}

Given the distance from a user to the BS as $d$, the channel gain $\|\mathbf{h}\|^{2}$ follows the Gamma distribution [90], whose conditional PDF and CDF are given by

$$
\begin{aligned}
& f_{\|\mathbf{h}\|^{2} \mid d}(x)=\operatorname{Gamma}\left(M, 1+d^{\alpha}, x\right) \text { and } \\
& F_{\|\mathbf{h}\|^{2} \mid d}(x)=\frac{\gamma_{L}\left(M,\left(1+d^{\alpha}\right) x\right)}{\Gamma(M)},
\end{aligned}
$$

respectively. Then, the CDF of the channel gain $\|\mathbf{h}\|^{2}$ can be obtained by

$$
F_{\|\mathbf{h}\|^{2}}(x)=\int_{D_{0}}^{D} \frac{\gamma_{L}\left(M,\left(1+d^{\alpha}\right) x\right)}{\Gamma(M)} f_{d}(z) d z .
$$

By applying the Gaussian-Chebyshev quadrature approximation in $(76)$, the CDF and PDF of $\|\mathbf{h}\|^{2}$ can be written as

$$
\begin{aligned}
& F_{\|\mathbf{h}\|^{2}}(x) \approx 1-\frac{1}{D+D_{0}} \sum_{n=1}^{N} \frac{\beta_{n} \gamma_{L}\left(M, c_{n} x\right)}{\Gamma(M)} \text { and } \\
& f_{\|\mathbf{h}\|^{2}}(x) \approx \frac{1}{D+D_{0}} \sum_{n=1}^{N} \beta_{n} \operatorname{Gamma}\left(M, c_{n}, x\right), x \geq 0,
\end{aligned}
$$

respectively, where $\beta_{n}$ and $c_{n}$ are given in 10 .

\section{Proof of Theorem 2}

Based on (87) in the proof of Theorem 1 in Appendix B, under the equal resource allocation strategy, i.e., $p_{k}=\frac{P_{\max }}{K}$, $\forall k$, the asymptotic individual rate of user $k$ of the $m$ MIMONOMA system with the MRC-SIC detection in (42) can be obtained by Equation (92) on the top of next page. With the aid of a large-scale antenna array, i.e., $M \rightarrow \infty$, the fluctuation imposed by the small-scale fading on the channel gain can be averaged out as a benefit of channel hardening [91]. Therefore, the channel gain is mainly determined by the large-scale fading asymptotically as follows:

$$
\lim _{M \rightarrow \infty} \frac{\left\|\mathbf{h}_{k}\right\|^{2}}{M}=\frac{1}{1+d_{k}^{\alpha}} .
$$

As a result, the asymptotic data rate of user $k$ in 92 is given by Equation 94 on the top of next page.

Based on the theory of order statistics [61], the PDF of $d_{k}$ is given by

$$
\begin{gathered}
f_{d_{k}}(x)=k\left(\begin{array}{c}
K \\
k
\end{array}\right) F_{d}^{k-1}(x)\left(1-F_{d}(x)\right)^{K-k} f_{d}(x), \\
D_{0} \leq z \leq D
\end{gathered}
$$

Thus, the mean of the large-scale fading of user $k$ can be written as

$$
\begin{aligned}
I_{k} & =\mathrm{E}_{d_{k}}\left\{\frac{1}{1+d_{k}^{\alpha}}\right\}=\int_{D_{0}}^{D} \frac{1}{1+x^{\alpha}} f_{d_{k}}(x) d x \\
& \approx\left(\begin{array}{c}
K \\
k
\end{array}\right) \frac{k}{D+D_{0}} \sum_{n=1}^{N} \frac{\beta_{n}}{c_{n}}\left(\frac{\phi_{n}^{2}-D_{0}^{2}}{D^{2}-D_{0}^{2}}\right)^{k-1}\left(\frac{D^{2}-\phi_{n}^{2}}{D^{2}-D_{0}^{2}}\right)^{K-k},
\end{aligned}
$$

with $\phi_{n}=\frac{D-D_{0}}{2} \cos \frac{2 n-1}{2 N} \pi+\frac{D+D_{0}}{2}$. For a large number of users, i.e., $K \rightarrow \infty$, the random IUI term in 94 can be approximated by a deterministic value given by

$$
\lim _{K \rightarrow \infty} \sum_{i=k+1}^{K} P_{\max } \frac{1}{1+d_{i}^{\alpha}} \approx \lim _{K \rightarrow \infty} \sum_{i=k+1}^{K} P_{\max } I_{i} .
$$

Now, the asymptotic ergodic data rate of user $k$ can be approximated by Equation (98) on the top of next page, where $\psi_{k}=\frac{P_{\max } M}{\sum_{i=k+1}^{K} P_{\max } I_{i}+K N_{0}}$. Substituting 98) into 433 yields the asymptotic ergodic sum-rate of the $m$ MIMO-NOMA system with the MRC-SIC detection as in (44), which completes the proof.

\section{E. Proof of Theorem 3}

With $D=D_{0}$, based on the channel hardening property [91], the channel gain can be asymptotically formulated as:

$$
\lim _{M \rightarrow \infty} \frac{\left\|\mathbf{h}_{k}\right\|^{2}}{M}=\frac{1}{1+D_{0}^{\alpha}}, \forall k .
$$

Substituting (99) into (92), the asymptotic individual rate of user $k$ of the mMIMO-NOMA system with $D=D_{0}$ is obtained by

$$
\begin{aligned}
& \lim _{K \rightarrow \infty, M \rightarrow \infty} R_{k}^{m \text { MIMO-NOMA }} \\
& =\lim _{K \rightarrow \infty, M \rightarrow \infty} \ln \left(1+M \frac{P_{\max } \frac{1}{1+D_{0}^{\alpha}}}{\sum_{i=k+1}^{K} P_{\max } \frac{1}{1+D_{0}^{\alpha}}+K N_{0}}\right) \\
& =\lim _{K \rightarrow \infty, M \rightarrow \infty} \ln \left(1+\frac{\delta \varpi}{\left(1-\frac{k}{K}\right) \varpi+1}\right)
\end{aligned}
$$

where $\delta=\frac{M}{K}$ and $\varpi=\frac{P_{\max }}{\left(1+D_{0}^{\alpha}\right) N_{0}}$. We can observe that the asymptotic individual rate of user $k$ in 100 becomes a deterministic value for $K \rightarrow \infty$ 


$$
\lim _{K \rightarrow \infty} R_{k}^{m \text { MIMO-NOMA }}=\lim _{K \rightarrow \infty} \ln \left(1+\frac{P_{\max }\left\|\mathbf{h}_{k}\right\|^{2}}{\sum_{i=k+1}^{K} P_{\max }\left\|\mathbf{h}_{i}\right\|^{2} \frac{1}{M}+K N_{0}}\right)
$$

$$
\lim _{K \rightarrow \infty, M \rightarrow \infty} R_{k}^{m \text { MIMO-NOMA }}=\lim _{K \rightarrow \infty, M \rightarrow \infty} \ln \left(1+\frac{M P_{\max } \frac{1}{1+d_{k}^{\alpha}}}{\sum_{i=k+1}^{K} P_{\max } \frac{1}{1+d_{i}^{\alpha}}+K N_{0}}\right)
$$

$$
\begin{aligned}
\lim _{K \rightarrow \infty, M \rightarrow \infty} \overline{R_{k}^{m \text { MIMO-NOMA }}} & \approx \lim _{K \rightarrow \infty, M \rightarrow \infty} \int_{D_{0}}^{D} \ln \left(1+\frac{\psi_{k}}{1+x^{\alpha}}\right) f_{d_{k}}(x) d x \\
& \approx \lim _{K \rightarrow \infty, M \rightarrow \infty}\left(\begin{array}{c}
K \\
k
\end{array}\right) \frac{k}{D+D_{0}} \sum_{n=1}^{N} \beta_{n} \ln \left(1+\frac{\psi_{k}}{c_{n}}\right)\left(\frac{\phi_{n}^{2}-D_{0}^{2}}{D^{2}-D_{0}^{2}}\right)^{k-1}\left(\frac{D^{2}-\phi_{n}^{2}}{D^{2}-D_{0}^{2}}\right)^{K-k}
\end{aligned}
$$

and $M \rightarrow \infty$ due to the channel hardening property. As a result, we have $\lim _{K \rightarrow \infty, M \rightarrow \infty} R_{k}^{m \text { MIMO-NOMA }}=$ $\lim _{K \rightarrow \infty, M \rightarrow \infty} \overline{R_{k}^{m \mathrm{MIMO}-\mathrm{NOMA}}}$.

Now, the asymptotic ergodic sum-rate of the $m$ MIMONOMA system with MRC-SIC receiver can be obtained by Equation 101 on the top of next page. which completes the proof of 48.

On the other hand, under the equal resource allocation strategy, the asymptotic individual rate of user $k$ of the $m$ MIMO-OMA system with the MRC detection in 46 can be approximated by

$$
R_{k}^{m \mathrm{MIMO}-\mathrm{OMA}} \approx \delta \varsigma \ln \left(1+\frac{P_{\max }\left\|\mathbf{h}_{k}\right\|^{2}}{\varsigma M N_{0}}\right) .
$$

Exploiting the channel hardening property as stated in (99), the individual rate of user $k$ in (102) can be approximated by a deterministic value and we have the asymptotic ergodic sum-rate of the mMIMO-OMA system considered as

$$
\lim _{M \rightarrow \infty} \overline{R_{\text {sum }}^{m \mathrm{MIMO}-\mathrm{OMA}}} \approx \lim _{M \rightarrow \infty} \varsigma M \ln \left(1+\frac{\varpi}{\varsigma}\right),
$$

which completes the proof of 49 .

\section{F. Derivations of Equation (73)}

Based on Equation (1), the residual signal in the $k$-th step of the MMSE-SIC detection is given by

$$
\mathbf{y}_{k}=\underbrace{\mathbf{h}_{k} \sqrt{p_{k}} x_{k}}_{\text {Desired signal }}+\underbrace{\sum_{i=k+1}^{K} \mathbf{h}_{i} \sqrt{p_{i}} x_{i}}_{\text {IUI }}+\underbrace{\sum_{i=1}^{k-1} \eta_{i} \mathbf{h}_{i} \sqrt{p_{i}} x_{i}}_{\text {Residual interference }}+\mathbf{v}
$$

where the first term is the desired signal of user $k$, the second term denotes the IUI originating from the remaining users $k+1, \ldots, K$ during the MMSE-SIC detection, and the third term is the residual interference of the preceding MMSE-SIC detection for users $1, \ldots, k-1$. According to [27], the SINR of the MMSE estimator for $x_{k}$ is given by Equation 105 on the top of next page. Then, following the matrix inversion lemma introduced in footnote 13 , the instantaneous achievable data rate of user $k$ of the MIMO-NOMA systems associated with imperfect SIC is given by Equation 106 on the top of next page. Note that when $\eta_{1}=\eta_{2}, \ldots, \eta_{K}=0$, Equation (106) degenerates to Equation [19], which is for the case of perfect SIC detection.

\section{G. Remark on Adopting Independently Distributed Channels in Analysis}

We note that channel ordering introduces channel correlation among the ordered users within a NOMA cluster [61]. However, the correlation introduced by channel ordering is not taken into account in our performance analysis. In the following, let us clarify the reasons for adopting independently distributed channels for our analysis in this work.

Firstly, as shown in Equations (6) and (20), the instantaneous sum-rates of both the SISO-NOMA and MIMONOMA systems are actually independent of the channel order. Furthermore, both the SISO-OMA and MIMO-OMA systems adopted a random user grouping strategy and hence their instantaneous sum-rates in Equations (7), 27, and (30) are also independent of the channel ordering. Therefore, we do not need to consider the channel ordering in single-antenna as well as multi-antenna systems and analyze their ergodic sumrate gains by treating the users' channels as i.i.d. as in Lemma 1 and Lemma 2 of the paper.

Secondly, as the instantaneous sum-rate of the mMIMONOMA system considered in Equation (43) does depend on the channel ordering, we analyze the ergodic sum-rate of $m$ MIMO-NOMA via employing order statistics, as derived in Appendix D. According to Equation 95 in Appendix D, the instantaneous data rate of user $k$ can be asymptotically approximated as a function of the random distance $d_{k}$ between user $k$ and the BS, i.e., $\lim _{K \rightarrow \infty, M \rightarrow \infty} R_{k}^{m \text { MIMO-NOMA }}\left(d_{k}\right)$. Additionally, as derived in [61], the joint distribution of all 


$$
\begin{aligned}
& \lim _{K \rightarrow \infty, M \rightarrow \infty} \overline{R_{\mathrm{sum}}^{m \mathrm{MIMO}-\mathrm{NOMA}}}=\lim _{K \rightarrow \infty, M \rightarrow \infty} \sum_{k=1}^{K} \ln \left(1+\frac{\delta \varpi}{\left(1-\frac{k}{K}\right) \varpi+1}\right)=\lim _{K \rightarrow \infty, M \rightarrow \infty} K \int_{0}^{1} \ln \left(1+\frac{\delta \varpi}{(1-x) \varpi+1}\right) d x \\
& =\lim _{K \rightarrow \infty, M \rightarrow \infty} \frac{M}{\varpi \delta}[\ln (1+\varpi \delta+\varpi)(1+\varpi \delta+\varpi)-\ln (1+\varpi \delta)(1+\varpi \delta)-\ln (1+\varpi)(1+\varpi)]
\end{aligned}
$$

$$
\operatorname{SINR}_{k}=\frac{p_{k}}{N_{0}} \mathbf{h}_{k}^{\mathrm{H}}\left(\mathbf{I}_{M}+\frac{1}{N_{0}} \sum_{i=k}^{K} p_{i} \mathbf{h}_{i} \mathbf{h}_{i}^{\mathrm{H}}+\frac{1}{N_{0}} \sum_{i=1}^{k-1} \eta_{i} p_{i} \mathbf{h}_{i} \mathbf{h}_{i}^{\mathrm{H}}\right)^{-1} \mathbf{h}_{k}
$$

$$
\begin{aligned}
R_{k}^{\mathrm{MIMO}-\mathrm{NOMA}} & =\ln \left(1+\mathrm{SINR}_{k}\right) \\
& =\ln \left|\mathbf{I}_{M}+\frac{1}{N_{0}} \sum_{i=k}^{K} p_{i} \mathbf{h}_{i} \mathbf{h}_{i}^{\mathrm{H}}+\frac{1}{N_{0}} \sum_{i=1}^{k-1} \eta_{i} p_{i} \mathbf{h}_{i} \mathbf{h}_{i}^{\mathrm{H}}\right|-\ln \left|\mathbf{I}_{M}+\frac{1}{N_{0}} \sum_{i=k+1}^{K} p_{i} \mathbf{h}_{i} \mathbf{h}_{i}^{\mathrm{H}}+\frac{1}{N_{0}} \sum_{i=1}^{k-1} \eta_{i} p_{i} \mathbf{h}_{i} \mathbf{h}_{i}^{\mathrm{H}}\right|
\end{aligned}
$$

the ordered $d_{k}$ is given by

$$
f_{d_{1}, \ldots, d_{K}}\left(x_{1}, \ldots, x_{K}\right)=K ! f_{d_{1}}\left(x_{1}\right) \ldots f_{d_{K}}\left(x_{K}\right) .
$$

Therefore, we can obtain the ergodic data rate

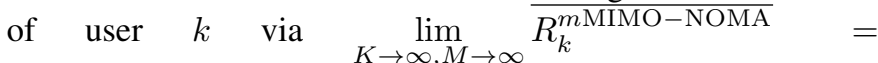
$\int \lim _{K \rightarrow \infty, M \rightarrow \infty} R_{k}^{m \text { MIMO-NOMA }}(x) f_{d_{k}}(x) d x \quad$ as derived in Equation [95), where $f_{d_{k}}(x)$ denotes the probability density function of $d_{k}$. Additionally, since a random grouping strategy is adopted for the mMIMO-OMA system considered, its ergodic sum-rate is also independent of the channel ordering. As a result, we can perform the ESG analysis of massive-MIMO systems without taking into account the channel correlation caused by channel ordering.

Thirdly, the inter-cell interference power derived in Equations (61) and (64) for multi-cell NOMA and multi-cell OMA systems, respectively, are irrelevant to the channel ordering in all the adjacent cells. Therefore, we do not need to consider the channel correlation introduced by channel ordering in the ESG analysis of multi-cell systems either.

\section{REFERENCES}

[1] Z. Wei, L. Yang, D. W. K. Ng, and J. Yuan, "On the performance gain of NOMA over OMA in uplink single-cell systems," in Proc. IEEE Global Commun. Conf., Dec. 2018, pp. 1-7.

[2] J. Andrews, S. Buzzi, W. Choi, S. Hanly, A. Lozano, A. Soong, and J. Zhang, "What will 5G be?" IEEE J. Select. Areas Commun., vol. 32, no. 6, pp. 1065-1082, Jun. 2014.

[3] V. W. Wong, R. Schober, D. W. K. Ng, and L.-C. Wang, Key Technologies for 5G Wireless Systems. Cambridge University Press, 2017.

[4] M. Zorzi, A. Gluhak, S. Lange, and A. Bassi, "From today's INTRAnet of things to a future INTERnet of things: a wireless- and mobility-related view," IEEE Wireless Commun. Mag., vol. 17, no. 6, pp. 44-51, Dec. 2010.

[5] "The 5G unified air interface: Scalable to an extreme variation of requirements," Qualcomm Technologies Inc., Tech. Rep., Nov. 2015.

[6] Z. Sun, Y. Xie, J. Yuan, and T. Yang, "Coded slotted ALOHA for erasure channels: Design and throughput analysis," IEEE Trans. Commun., vol. 65 , no. 11, Nov. 2017.

[7] Z. Sun, Z. Wei, L. Yang, J. Yuan, X. Cheng, and L. Wan, "Joint user identification and channel estimation in massive connectivity with transmission control," in Proc. IEEE Intern. Sympos. on Turbo Codes Iterative Information Process., 2018, pp. 1-5.
[8] T. Marzetta, "Noncooperative cellular wireless with unlimited numbers of base station antennas," IEEE Trans. Wireless Commun., vol. 9, no. 11, pp. 3590-3600, Nov. 2010.

[9] H. Q. Ngo, E. G. Larsson, and T. L. Marzetta, "Energy and spectral efficiency of very large multiuser MIMO systems," IEEE Trans. Commun., vol. 61, no. 4, pp. 1436-1449, Apr. 2013.

[10] L. Zhao, J. Guo, Z. Wei, D. W. K. Ng, and J. Yuan, "A distributed multiRF chain hybrid mmwave scheme for small-cell systems," in Proc. IEEE Intern. Commun. Conf., May 2019, pp. 1-7.

[11] Z. Wei, M. Qiu, D. W. Kwan Ng, and J. Yuan, "A two-stage beam alignment framework for hybrid mmwave distributed antenna systems," in Proc. IEEE Intern. Workshop on Signal Process. Advances in Wireless Commun., Jul. 2019, pp. 1-5.

[12] L. Dai, B. Wang, Y. Yuan, S. Han, I. Chih-Lin, and Z. Wang, "Nonorthogonal multiple access for $5 \mathrm{G}$ : solutions, challenges, opportunities, and future research trends," IEEE Commun. Mag., vol. 53, no. 9, pp 74-81, Sep. 2015.

[13] Z. Ding, Y. Liu, J. Choi, Q. Sun, M. Elkashlan, C. L. I, and H. V. Poor, "Application of non-orthogonal multiple access in LTE and 5G networks," IEEE Commun. Mag., vol. 55, no. 2, pp. 185-191, Feb. 2017.

[14] Z. Wei, Y. Jinhong, D. W. K. Ng, M. Elkashlan, and Z. Ding, "A survey of downlink non-orthogonal multiple access for $5 \mathrm{G}$ wireless communication networks," ZTE Commun., vol. 14, no. 4, pp. 17-25, Oct. 2016.

[15] M. Qiu, Y. Huang, S. Shieh, and J. Yuan, "A lattice-partition framework of downlink non-orthogonal multiple access without SIC," IEEE Trans. Commun., vol. 66, no. 6, pp. 2532-2546, Jun. 2018.

[16] M. Qiu, Y. Huang, J. Yuan, and C. Wang, "Lattice-partition-based downlink non-orthogonal multiple access without SIC for slow fading channels," IEEE Trans. Commun., vol. 67, no. 2, pp. 1166-1181, Feb. 2019.

[17] T. Rappaport, S. Sun, R. Mayzus, H. Zhao, Y. Azar, K. Wang, G. Wong, J. Schulz, M. Samimi, and F. Gutierrez, "Millimeter wave mobile communications for 5G cellular: It will work!" IEEE Access, vol. 1, pp. 335-349, May 2013.

[18] M. Xiao, S. Mumtaz, Y. Huang, L. Dai, Y. Li, M. Matthaiou, G. K. Karagiannidis, E. Björnson, K. Yang, C. L. I, and A. Ghosh, "Millimeter wave communications for future mobile networks," IEEE J. Select. Areas Commun., vol. 35, no. 9, pp. 1909-1935, Sep. 2017.

[19] L. Zhao, D. W. K. Ng, and J. Yuan, "Multi-user precoding and channel estimation for hybrid millimeter wave systems," IEEE J. Select. Areas Commun., vol. 35, no. 7, pp. 1576-1590, Jul. 2017.

[20] Z. Wei, L. Zhao, J. Guo, D. W. K. Ng, and J. Yuan, "Multi-beam NOMA for hybrid mmwave systems," IEEE Trans. Commun., vol. 67, no. 2, pp. 1705-1719, Feb. 2019.

[21] Z. Wei, D. W. Kwan Ng, and J. Yuan, "NOMA for hybrid mmwave communication systems with beamwidth control," IEEE J. Select. Topics Signal Process., vol. 13, no. 3, pp. 567-583, Jun. 2019.

[22] Y. Chen, A. Bayesteh, Y. Wu, B. Ren, S. Kang, S. Sun, Q. Xiong, C. Qian, B. Yu, Z. Ding, S. Wang, S. Han, X. Hou, H. Lin, R. Visoz, and R. Razavi, "Toward the standardization of non-orthogonal multiple 
access for next generation wireless networks," IEEE Commun. Mag., vol. 56, no. 3, pp. 19-27, Mar. 2018.

[23] D. W. K. Ng, E. S. Lo, and R. Schober, "Energy-efficient resource allocation in OFDMA systems with large numbers of base station antennas," IEEE Trans. Wireless Commun., vol. 11, no. 9, pp. 3292 3304, Sep. 2012.

[24] — , "Energy-efficient resource allocation in OFDMA systems with large numbers of base station antennas," IEEE Transactions on Wireless Communications, vol. 11, no. 9, pp. 3292-3304, Sep. 2012.

[25] —, "Wireless information and power transfer: Energy efficiency optimization in OFDMA systems," IEEE Trans. Wireless Commun., vol. 12, no. 12, pp. 6352-6370, Dec. 2013.

[26] T. M. Cover and J. A. Thomas, Elements of Information Theory. John Wiley \& Sons, Inc., 1991.

[27] D. Tse and P. Viswanath, Fundamentals of wireless communication. Cambridge university press, 2005.

[28] S. Verdu and S. Shamai, "Spectral efficiency of CDMA with random spreading," IEEE Trans. Inf. Theory, vol. 45, no. 2, pp. 622-640, Mar. 1999.

[29] L.-L. Yang and L. Hanzo, "Multicarrier DS-CDMA: A multiple access scheme for ubiquitous broadband wireless communications," IEEE Commun. Mag., pp. 116-124, Oct. 2003.

[30] L. Hanzo, L.-L. Yang, E. Kuan, and K. Yen, Single- and Multi-Carrier DS-CDMA: Multi-USer Detection, Space-Time Spreading, Synchronisation, Standards and Networking. John Wiley \& Sons, Aug. 2003.

[31] P. Wang, J. Xiao, and P. Li, "Comparison of orthogonal and nonorthogonal approaches to future wireless cellular systems," IEEE Veh. Technol. Mag., vol. 1, no. 3, pp. 4-11, Sep. 2006.

[32] P. Li, L. Liu, K. Wu, and W. K. Leung, "Interleave division multipleaccess," IEEE Trans. Wireless Commun., vol. 5, no. 4, pp. 938-947, Apr. 2006.

[33] "Study on downlink multiuser supersition transmission (MUST) for LTE (Release 13)," 3GPP TR 36.859, Tech. Rep., Dec. 2015.

[34] DOCOMO and MediaTek. (2017) DOCOMO and MediaTek achieve world's first successful 5G trial using smartphone-sized NOMA chipset-embedded device to increase spectral efficiency. [Online]. Available: https://www.nttdocomo.co.jp/english/info/media_ center/pr/2017/1102_02.html

[35] C. Xu, Y. Hu, C. Liang, J. Ma, and P. Li, "Massive MIMO, nonorthogonal multiple access and interleave division multiple access," IEEE Access, vol. 5, pp. 14728-14748, Jul. 2017.

[36] Y. Liu, Z. Qin, M. Elkashlan, Z. Ding, A. Nallanathan, and L. Hanzo, "Nonorthogonal multiple access for 5G and beyond," Proceedings of the IEEE, vol. 105, no. 12, pp. 2347-2381, Dec. 2017.

[37] L. Dai, B. Wang, Z. Ding, Z. Wang, S. Chen, and L. Hanzo, "A survey of non-orthogonal multiple access for 5G," IEEE Commun. Surveys Tuts. Mag., vol. 20, no. 3, pp. 2294-2323, May 2018

[38] P. Xu, Z. Ding, X. Dai, and H. V. Poor, "A new evaluation criterion for non-orthogonal multiple access in 5G software defined networks," IEEE Access, vol. 3, pp. 1633-1639, Sep. 2015.

[39] Z. Ding, Z. Yang, P. Fan, and H. Poor, "On the performance of nonorthogonal multiple access in 5G systems with randomly deployed users," IEEE Signal Process. Lett., vol. 21, no. 12, pp. 1501-1505, Dec. 2014.

[40] Z. Chen, Z. Ding, X. Dai, and R. Zhang, "An optimization perspective of the superiority of NOMA compared to conventional OMA," IEEE Trans. Signal Process., vol. 65, no. 19, pp. 5191-5202, Oct. 2017.

[41] Z. Yang, Z. Ding, P. Fan, and G. K. Karagiannidis, "On the performance of non-orthogonal multiple access systems with partial channel information," IEEE Trans. Commun., vol. 64, no. 2, pp. 654-667, Feb. 2016.

[42] Y. Sun, D. W. K. Ng, Z. Ding, and R. Schober, "Optimal joint power and subcarrier allocation for full-duplex multicarrier non-orthogonal multiple access systems," IEEE Trans. Commun., vol. 65, no. 3, pp. 1077-1091, Mar. 2017.

[43] Z. Wei, D. W. K. Ng, J. Yuan, and H. M. Wang, "Optimal resource allocation for power-efficient MC-NOMA with imperfect channel state information," IEEE Trans. Commun., vol. 65, no. 9, pp. 3944-3961, May 2017.

[44] Z. Ding, R. Schober, and H. V. Poor, "A general MIMO framework for NOMA downlink and uplink transmission based on signal alignment," IEEE Trans. Wireless Commun., vol. 15, no. 6, pp. 4438-4454, Jun. 2016.

[45] Z. Chen, Z. Ding, X. Dai, and G. K. Karagiannidis, "On the application of quasi-degradation to MISO-NOMA downlink," IEEE Trans. Signal Process., vol. 64, no. 23, pp. 6174-6189, Dec. 2016.
[46] M. Zeng, A. Yadav, O. A. Dobre, G. I. Tsiropoulos, and H. V. Poor, "Capacity comparison between MIMO-NOMA and MIMO-OMA with multiple users in a cluster," IEEE J. Select. Areas Commun., vol. 35, no. 10, pp. 2413-2424, Oct. 2017.

[47] Y. Liu, G. Pan, H. Zhang, and M. Song, "On the capacity comparison between MIMO-NOMA and MIMO-OMA," IEEE Access, vol. 4, pp. 2123-2129, May 2016

[48] Z. Ding and H. V. Poor, "Design of Massive-MIMO-NOMA with limited feedback," IEEE Signal Process. Lett., vol. 23, no. 5, pp. 629-633, May 2016.

[49] L. You, D. Yuan, L. Lei, S. Sun, S. Chatzinotas, and B. Ottersten, "Resource optimization with load coupling in multi-cell NOMA," IEEE Trans. Wireless Commun., vol. 17, no. 7, pp. 4735-4749, Jul. 2018.

[50] Y. Fu, Y. Chen, and C. W. Sung, "Distributed power control for the downlink of multi-cell NOMA systems," IEEE Trans. Wireless Commun., vol. 16, no. 9, pp. 6207-6220, Sep. 2017.

[51] V. Nguyen, H. D. Tuan, T. Q. Duong, H. V. Poor, and O. Shin, "Precoder design for signal superposition in MIMO-NOMA multicell networks," IEEE J. Select. Areas Commun., vol. 35, no. 12, pp. 2681-2695, Dec. 2017.

[52] Y. Sun, D. W. K. Ng, J. Zhu, and R. Schober, "Robust and secure resource allocation for full-duplex MISO multicarrier NOMA systems," IEEE Trans. Commun., vol. 66, no. 9, pp. 4119-4137, Sep. 2018.

[53] D. Zhang, K. Yu, Z. Wen, and T. Sato, "Outage probability analysis of NOMA within massive MIMO systems," in Proc. IEEE Veh. Techn. Conf., May 2016, pp. 1-5.

[54] D. Zhang, Z. Zhou, C. Xu, Y. Zhang, J. Rodriguez, and T. Sato, "Capacity analysis of NOMA with mmWave massive MIMO systems," IEEE J. Select. Areas Commun., vol. 35, no. 7, pp. 1606-1618, Jul. 2017.

[55] W. Shin, M. Vaezi, B. Lee, D. J. Love, J. Lee, and H. V. Poor, "Nonorthogonal multiple access in multi-cell networks: Theory, performance, and practical challenges," IEEE Commun. Mag., vol. 55, no. 10, pp. 176-183, Oct. 2017.

[56] M. Abramowitz and I. A. Stegun, Handbook of mathematical functions: with formulas, graphs, and mathematical tables. Courier Corporation, 1964 , vol. 55.

[57] Z. Wei, J. Guo, D. W. K. Ng, and J. Yuan, "Fairness comparison of uplink NOMA and OMA," in Proc. IEEE Veh. Techn. Conf., 2017, pp. $1-6$.

[58] "Evolved universal terrestrial radio access: Further advancements for E-UTRA physical layer aspects," 3GPP TR 36.814, Tech. Rep., 2010.

[59] S. Vishwanath, N. Jindal, and A. Goldsmith, "Duality, achievable rates, and sum-rate capacity of Gaussian MIMO broadcast channels," IEEE Trans. Inf. Theory, vol. 49, no. 10, pp. 2658-2668, Oct. 2003.

[60] P. Wang and P. Li, "On maximum eigenmode beamforming and multiuser gain," IEEE Trans. Inf. Theory, vol. 57, no. 7, pp. 4170-4186, Jul. 2011.

[61] H. A. David and H. N. Nagaraja, "Order statistics," NJ: John Wiley \& Sons, vol. 7, pp. 159-61, 2003.

[62] Z. Ding, P. Fan, and H. V. Poor, "Impact of user pairing on 5G nonorthogonal multiple-access downlink transmissions," IEEE Trans. Veh. Technol., vol. 65, no. 8, pp. 6010-6023, Aug. 2016.

[63] Z. Ding, F. Adachi, and H. V. Poor, "The application of MIMO to nonorthogonal multiple access," IEEE Trans. Wireless Commun., vol. 15, no. 1 , pp. 537-552, Jan. 2016.

[64] L. Zheng and D. N. C. Tse, "Diversity and multiplexing: a fundamental tradeoff in multiple-antenna channels," IEEE Trans. Inf. Theory, vol. 49, no. 5, pp. 1073-1096, May 2003.

[65] M. Biguesh and A. B. Gershman, "Training-based MIMO channel estimation: a study of estimator tradeoffs and optimal training signals," IEEE Trans. Signal Process., vol. 54, no. 3, pp. 884-893, Mar. 2006.

[66] Z. Wei, D. W. K. Ng, and J. Yuan, "Joint pilot and payload power control for uplink MIMO-NOMA with MRC-SIC receivers," IEEE Commun. Lett., vol. 22, no. 4, pp. 692-695, Apr. 2018.

[67] Z. Wei, L. Dai, D. W. K. Ng, and J. Yuan, "Performance analysis of a hybrid downlink-uplink cooperative NOMA scheme," in Proc. IEEE Veh. Techn. Conf., Jun. 2017, pp. 1-7.

[68] H. Q. Ngo, E. G. Larsson, and T. L. Marzetta, "Energy and spectral efficiency of very large multiuser MIMO systems," IEEE Trans. Commun., vol. 61, no. 4, pp. 1436-1449, Apr. 2013.

[69] J. G. Andrews, "Interference cancellation for cellular systems: a contemporary overview," IEEE Wireless Commun. Mag., vol. 12, no. 2, pp. 19-29, Apr. 2005

[70] M. Vaezi, R. Schober, Z. Ding, and H. V. Poor, "Non-orthogonal multiple access: Common myths and critical questions," IEEE Wireless Commun. Mag., pp. 1-7, early access 2019. 
[71] W. Yu, W. Rhee, S. Boyd, and J. Cioffi, "Iterative water-filling for Gaussian vector multiple-access channels," IEEE Trans. Inf. Theory, vol. 50, no. 1, pp. 145-152, Jan. 2004.

[72] M. Al-Imari, P. Xiao, M. A. Imran, and R. Tafazolli, "Uplink nonorthogonal multiple access for 5G wireless networks," in Proc. IEEE Intern. Sympos. on Wireless Commun. Systems, Aug. 2014, pp. 781785 .

[73] N. Zhang, J. Wang, G. Kang, and Y. Liu, "Uplink non-orthogonal multiple access in 5G systems," IEEE Commun. Lett., vol. 20, no. 3, pp. 458-461, Mar. 2016.

[74] E. Balevi and R. D. Gitlin, "Pareto optimization for uplink NOMA power control," in Proc. Wireless Telecomm. Sympos., Apr. 2018, pp. 1-5.

[75] Z. Sun, Z. Wei, L. Yang, J. Yuan, X. Cheng, and L. Wan, "Exploiting transmission control for joint user identification and channel estimation in massive connectivity," IEEE Trans. Commun., vol. 67, no. 9, pp. 6311-6326, Sep. 2019.

[76] K. E. Atkinson, An introduction to numerical analysis. John Wiley \& Sons, 2008.

[77] R. G. Bartle, The elements of integration and Lebesgue measure. John Wiley \& Sons, Inc., 2014.

[78] K. Kedlaya, "Proof of a mixed arithmetic-mean, geometric-mean inequality," The American Mathematical Monthly, vol. 101, no. 4, pp. 355-357, 1994.

[79] E. Balevi, F. T. A. Rabee, and R. D. Gitlin, "ALOHA-NOMA for massive machine-to-machine IoT communication," in Proc. IEEE Intern. Commun. Conf., May 2018, pp. 1-5.

[80] A. Shahini and N. Ansari, "NOMA aided narrowband IoT for machine type communications with user clustering," IEEE Internet Things J., vol. 6, no. 4, pp. 7183-7191, Aug. 2019.

[81] G. Caire, R. R. Muller, and R. Knopp, "Hard fairness versus proportional fairness in wireless communications: The single-cell case," IEEE Trans. Inf. Theory, vol. 53, no. 4, pp. 1366-1385, Apr. 2007.

[82] Z. Ding, M. Peng, and H. Poor, "Cooperative non-orthogonal multiple access in 5G systems," IEEE Commun. Lett., vol. 19, no. 8, pp. 1462 1465, Aug. 2015.

[83] H. Tabassum, E. Hossain, and J. Hossain, "Modeling and analysis of uplink non-orthogonal multiple access in large-scale cellular networks using poisson cluster processes," IEEE Trans. Commun., vol. 65, no. 8, pp. 3555-3570, Aug. 2017.

[84] A. Goldsmith, S. A. Jafar, N. Jindal, and S. Vishwanath, "Capacity limits of MIMO channels," IEEE J. Select. Areas Commun., vol. 21, no. 5, pp. 684-702, Jun. 2003

[85] R. W. Heath, T. Wu, Y. H. Kwon, and A. C. K. Soong, "Multiuser MIMO in distributed antenna systems with out-of-cell interference," IEEE Trans. Signal Process., vol. 59, no. 10, pp. 4885-4899, Oct. 2011.

[86] L. Zhao, Z. Wei, D. W. K. Ng, J. Yuan, and M. C. Reed, "Multi-cell hybrid millimeter wave systems: Pilot contamination and interference mitigation," IEEE Trans. Commun., vol. 66, no. 11, pp. 5740-5755, Nov. 2018.

[87] M. Haenggi, J. G. Andrews, F. Baccelli, O. Dousse, and M. Franceschet$\mathrm{ti}$, "Stochastic geometry and random graphs for the analysis and design of wireless networks," IEEE J. Select. Areas Commun., vol. 27, no. 7, pp. 1029-1046, Sep. 2009.

[88] X. Chen, R. Jia, and D. W. K. Ng, "On the design of massive non-orthogonal multiple access with imperfect successive interference cancellation," IEEE Trans. Commun., vol. 67, no. 3, pp. 2539-2551, Mar. 2019.

[89] H. Sun, B. Xie, R. Q. Hu, and G. Wu, "Non-orthogonal multiple access with SIC error propagation in downlink wireless MIMO networks," in Proc. IEEE Veh. Techn. Conf., 2016, pp. 1-5.

[90] Q. Yang, H.-M. Wang, D. W. K. Ng, and M. H. Lee, "NOMA in downlink SDMA with limited feedback: Performance analysis and optimization," IEEE J. Select. Areas Commun., vol. 35, no. 10, pp. 22812294, Oct. 2017.

[91] B. M. Hochwald, T. L. Marzetta, and V. Tarokh, "Multiple-antenna channel hardening and its implications for rate feedback and scheduling," IEEE Trans. Inf. Theory, vol. 50, no. 9, pp. 1893-1909, Sep. 2004.

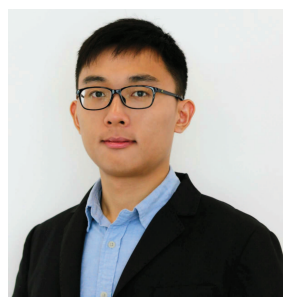

Zhiqiang Wei (S'16-M'19) received the B.E. degree in information engineering from the Northwestern Polytechnical University (NPU), Xi'an, China, in 2012 and the Ph.D. degree in Electrical Engineering and Telecommunications from the University of New South Wales, Sydney, Australia, in 2019. $\mathrm{He}$ is currently a postdoc research fellow in the University of New South Wales, Sydney, Australia. $\mathrm{He}$ received the Best Paper Awards at the IEEE International Conference on Communications (ICC), 2018. His current research interests include statistic and array signal processing, non-orthogonal multiple access, millimeter wave communications, and resource allocation design.

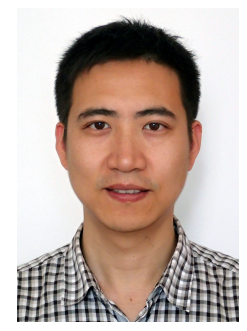

Lei Yang received the B.E. and M.Sc. degrees in electronics engineering from Beihang University, Beijing, China, in 2004 and 2007, respectively, and the Ph.D. degree in communications and information system from Beijing Institute of Technology, Beijing, China, in 2015. He received the Australian Research Council (ARC) Discovery Early Career Researcher Award (DECRA) in 2019. He is currently a professor at the Technology and Engineering Center for Space Utilization of Chinese Academy of Sciences. His current research interests include free-space optical communications, error control coding, spectrum-efficient coded modulation and iterative signal processing.

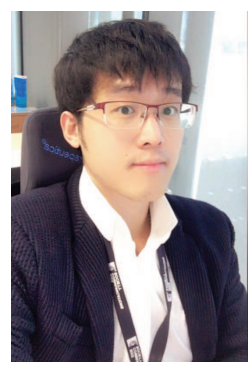

Derrick Wing Kwan Ng (S'06-M'12-SM'17) received the bachelor degree with first-class honors and the Master of Philosophy (M.Phil.) degree in electronic engineering from the Hong Kong University of Science and Technology (HKUST) in 2006 and 2008, respectively. He received his Ph.D. degree from the University of British Columbia (UBC) in 2012. He was a senior postdoctoral fellow at the Institute for Digital Communications, Friedrich-Alexander-University ErlangenNürnberg (FAU), Germany. He is now working as a Senior Lecturer and an ARC DECRA Research Fellow at the University of New South Wales, Sydney, Australia. His research interests include convex and non-convex optimization, physical layer security, wireless information and power transfer, and green (energy-efficient) wireless communications. Dr. $\mathrm{Ng}$ received the Best Paper Awards at the IEEE TCGCC Best Journal Paper Award 2018, INISCOM 2018, IEEE International Conference on Communications (ICC) 2018, IEEE International Conference on Computing, Networking and Communications (ICNC) 2016, IEEE Wireless Communications and Networking Conference (WCNC) 2012, the IEEE Global Telecommunication Conference (Globecom) 2011, and the IEEE Third International Conference on Communications and Networking in China 2008. He has been serving as an editorial assistant to the Editor-in-Chief of the IEEE Transactions on Communications since Jan. 2012. In addition, he is listed as a Highly Cited Researcher by Clarivate Analytics in 2018 and 2019. 


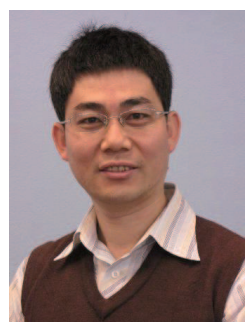

Jinhong Yuan (M'02-SM'11-F'16) received the B.E. and Ph.D. degrees in electronics engineering from the Beijing Institute of Technology, Beijing, China, in 1991 and 1997, respectively. From 1997 to 1999 , he was a Research Fellow with the School of Electrical Engineering, University of Sydney, Sydney, Australia. In 2000, he joined the School of Electrical Engineering and Telecommunications, University of New South Wales, Sydney, Australia, where he is currently a Professor and Head of Telecommunication Group with the School. He has published two books, five book chapters, over 300 papers in telecommunications journals and conference proceedings, and 50 industrial reports. $\mathrm{He}$ is a co-inventor of one patent on MIMO systems and two patents on lowdensity-parity-check codes. He has co-authored four Best Paper Awards and one Best Poster Award, including the Best Paper Award from the IEEE International Conference on Communications, Kansas City, USA, in 2018, the Best Paper Award from IEEE Wireless Communications and Networking Conference, Cancun, Mexico, in 2011, and the Best Paper Award from the IEEE International Symposium on Wireless Communications Systems, Trondheim, Norway, in 2007. He is an IEEE Fellow and currently serving as an Associate Editor for the IEEE Transactions on Wireless Communications. He served as the IEEE NSW Chapter Chair of Joint Communications/Signal Processions/Ocean Engineering Chapter during 2011-2014 and served as an Associate Editor for the IEEE Transactions on Communications during 2012-2017. His current research interests include error control coding and information theory, communication theory, and wireless communications.

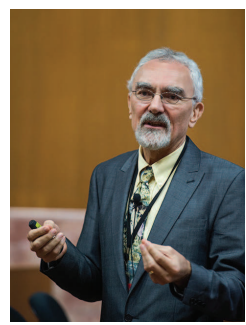

Lajos Hanzo (M91SM92F04) FREng, FIEEE, FIET, Fellow of EURASIP, DSc received his degree in electronics in 1976 and his doctorate in 1983. In 2009 he was awarded the honorary doctorate "Doctor Honaris Causa" by the Technical University of Budapest. During his 40-year career in telecommunications he has held various research and academic posts in Hungary, Germany and the UK. Since 1986, he has been with the School of Electronics and Computer Science, University of Southampton, UK, where he holds the chair in telecommunications. He has successfully supervised $119 \mathrm{PhD}$ students, co-authored $18 \mathrm{John}$ Wiley/IEEE Press books on mobile radio communications totalling in excess of 10000 pages, published 1880 research contributions at IEEE Xplore, acted both as TPC and General Chair of IEEE conferences, presented keynote lectures and has been awarded a number of distinctions. Currently he is directing an academic research team, working on a range of research projects in the field of wireless multimedia communications sponsored by industry, the Engineering and Physical Sciences Research Council (EPSRC) UK, the European IST Programme and the Mobile Virtual Centre of Excellence (VCE), UK. He is an enthusiastic supporter of industrial and academic liaison and he offers a range of industrial courses. He is also a Governor of the IEEE VTS. During 2008 - 2012 he was the Editor-in-Chief of the IEEE Press and since 2009 he has been a Chaired Professor also at Tsinghua University, Beijing. For further information on research in progress and associated publications please refer to http://www-mobile.ecs.soton.ac.uk 